\title{
Random versus Directed Search for Scarce Resources
}

\author{
Teddy Mekonnen
}

Current Draft: May 14, 2019

\begin{abstract}
This paper studies how different search protocols affect social welfare in a search market with scarcity. Agents search for objects that differ in quality either through a random or a directed search protocol. Random search protocol, in which agents are randomly paired to an object of any quality, gives rise to adversely selected markets. Directed search protocol, in which agents choose with which quality types to pair, gives rise to congestion. When utility is either non-transferable or transferable through Nash bargaining, I show that random search dominates directed search in terms of welfare, even though each agent would prefer to be able to direct her search.
\end{abstract}

JEL Classifications: C78, D62, D83

First Draft: December 20, 2018

Contact: mekonnen@caltech.edu. California Institute of Technology, SISL.

I would like to express my deepest appreciation to Eddie Dekel, Federico Echenique, Bruno Strulovici, and Asher Wolinsky for their guidance and mentorship. This work has also greatly benefited from conversations with Odilon Câmara, Hector Chade, Laura Doval, João Ramos, Jesse Shapiro, Pierre-Olivier Weill, and numerous seminar participants. 


\section{Introduction}

In this paper, I consider a simple search market in which agents search for objects. The market features scarcity, i.e., there are weakly more agents searching than there are objects available in the market. Additionally, the objects vary in quality so that some of the objects are desirable and some are not. The main result of the paper is that a search protocol that makes it is easy for agents to sort between the desirable and the undesirable objects actually lowers social welfare by generating congestion, even though an individual agent would prefer such a protocol. The model is applied to study a one-sided consumer search with non-transferable utility as well as two-sided labor search market with Nash bargaining.

To highlight the tension between an individual agent's preferences and social welfare, consider the following example. Suppose Jane just enrolled for health insurance. She wants to choose a high quality doctor as her primary care physician (PCP). From Jane's perspective, she is in a market without prices because her health expenses are covered by her insurance. She just has to search for the best "object" (doctor).

One possible search protocol for Jane is to randomly sample from the list of doctors covered by her network until she finds one that is high quality. I call this a random search protocol. Because Jane does not know a doctor's quality prior to sampling, she may have to search a long time.

There is another way Jane can search if she lives in California; she can use a ranking of doctors published by Consumer Reports magazine to sort through the doctors in her network. ${ }^{1}$ I call this a directed search protocol. In an isolated world where Jane is the only insurance enrollee, directed search is superior to random search because it allows Jane to instantly match with the best PCP.

However, in reality, many Californians enroll for health insurance, and each enrollee wants a high quality PCP. Additionally, it is unlikely that there are enough doctors to service all the enrollees which implies that doctors are a scarce resource. ${ }^{2}$ If enrollees are able to direct their search based on the Consumer Reports rankings, they would compete to match with the scarce number of high quality doctors leading to congestion. The main result of this paper shows that the congestion inefficiencies from directed search wipe out any informational benefit the enrollees get from using the Consumer Reports rankings. Ultimately, they would have been

\footnotetext{
${ }^{1}$ Consumer Reports publishes doctor and doctor-group rankings also in Maine, Michigan, Minnesota, Massachusetts, Ohio, Washington, and Wisconsin.

${ }^{2}$ The Association of American Medical Colleges document significant shortages in both primary care physicians and specialists. Dall et al. (2018)
} 
better off randomly sampling without any additional information about PCP quality.

Formally, I study how different search protocols affect welfare in a steady-state search market. One side of the market is composed of objects while the other side of the market is composed of rational and forward-looking agents. The basic search market has four characteristics: $(i)$ utility is non-transferable, $(i i)$ objects are a scarce resource, $($ iii $)$ the objects are vertically differentiated by quality and the agents have homogeneous preferences for higher quality objects, and $(i v)$ search is frictionless, i.e., the maximal number of agent-object pairs are formed. The first assumption is relaxed when I generalize the basic model to accommodate a labor search market with Nash bargaining. I discuss the implications of relaxing the last three assumptions later in the introduction and defer a formal treatment until Online Appendix A.

The first search protocol considered is random search in which agents are randomly paired to objects from the entire search market. If an agent is paired to an object, she observes the object's quality. The agent then either matches and exits the market with the object or declines to match. In the latter case, both the object and the agent return to the search market. A fraction $\gamma \in(0,1)$ of the agents and objects left in the search market "die," which can be thought of as the cost of searching. ${ }^{3}$ Finally, new agents and objects flow into the market with the quality of the newly arriving objects distributed according to some exogenous absolutely continuous distribution function $F$.

The second search protocol considered is directed search in which the entire search market is segmented by quality into smaller sub-markets. All objects contained in a sub-market have the same quality and all objects of the same quality are in the same sub-market. The agents can distinguish between the different sub-markets, i.e., the agents can use sub-markets to sort the objects by quality. In each period, an agent chooses which sub-market to enter. The agent is then randomly paired to an object within that sub-market with the short-side of the sub-market being rationed to the long-side. If an agent is paired to an object, she matches and exits the market. Before the period ends, a fraction $\gamma$ of the unmatched agents and objects left in the search market "die," and a mass of new agents and objects flow into the market.

Under a random search protocol, a stationary equilibrium is characterized by a quality cutoff such that an agent would rather continue searching than match with an object whose quality is below the cutoff. We can think of the cutoff as an agent's "pickiness." In the aggregate, the agents' pickiness gives rise to a negative externality in the form of an adversely selected market;

\footnotetext{
${ }^{3}$ The model can be modified to have different death probabilities for agents and objects without changing the main results as long as the scarcity assumption continues to hold. Effectively, $1-\gamma$ serves as a discount factor for the agents.
} 
the desirable high quality objects above the cutoff leave the market quickly while the rejected low quality objects below the cutoff linger in the market.

The main drawback of the random search protocol is that an agent is paired to a low quality object below her pickiness cutoff with positive probability. To make matters worse, the adversely selected market makes it more likely for an agent to pair with a low quality object than a high quality object. These inherent drawbacks of the random search protocol do not exist under a directed search protocol which enables the agent to sort the desirable high quality objects from the undesirable low quality objects. Can we then improve social welfare by changing the search protocol to directed search?

In the basic model of this paper, the answer is no. The main result states that social welfare is higher under random search than under directed search regardless of the search cost $\gamma$ or the distribution of quality $F$. Under directed search, the agents compete to match with the scarce number of high quality objects giving rise to congestion. Equilibrium is once again determined by a quality cutoff (pickiness) such that an agent would rather wait in a congested sub-market above the cutoff until a desirable high quality object is rationed to her instead of enter an un-congested sub-market below the cutoff that guarantees an immediate match with a low quality object.

Furthermore, the agents are pickier when they can direct their search than when they search randomly. Under random search, an agent's pickiness is characterized by an intertemporal trade-off between the object she can match with today and the possibly better objects she may get in the future if she continues to search at a cost. Under directed search, an agent's pickiness is characterized by a contemporaneous trade-off between the object she can get today with certainty and the possibly better objects she may get today through rationing. As the latter trade-off does not involve the search cost, the agents under directed search can afford to be pickier which makes the negative externalities they impose on the search market more severe. Ultimately, the additional information and flexibility under directed search leads to lower social welfare.

When the cost of searching $\gamma$ vanishes, so does the welfare gap between the two search protocols. Intuitively, when the cost of searching disappears, a random searcher is equivalent to a directed searcher; she can keep searching until she gets her desired quality without incurring any loss. Thus, welfare under random and directed search coincide in the limit. Importantly, the limit outcome as the search cost vanishes is inefficient.

The parsimonious model in this paper permits a tractable way of comparing the two search protocols without changing the underlying environment. While it is not tailored to a specific 
application, the basic model can be generalized to study a labor market in which firms play the role of the agents and workers of differing productivity play the role of the objects. In order to accommodate such a labor search market, I extend the model in two dimensions: First, both the firms and the workers are strategic with each side of the market looking to maximize payoffs. Second, utility is transferable in the form of wages that are endogenously determined on the equilibrium path through ex-post Nash Bargaining, as in Mortensen (1982), Diamond (1982), and Pissarides (1985).

As an example, consider the labor market for graduating college students. Under random search, a firm simply posts a vacancy. Once a student applies and has been interviewed (random pairing arrives and productivity is observed), the firm decides whether or not to hire the student at some bargained wage. There is a possibility that the vacancy remains unfilled if the student's productivity is too low. On the other hand, if students' productivity is correlated to the quality of the universities they attend, then each university can be seen as a sub-market. Under directed search, a firm can choose to recruit only the students of a specific university (e.g. campus career fairs). However, there may be a possibility that a vacancy goes unfilled because too many other firms also recruit at the same university.

Similar to the basic model with non-transferable utility, when utility is transferable through wages determined by the Nash bargaining solution, there exist equilibrium outcomes in directed search markets with a lower social welfare than in random search markets. The result stands in stark contrast to the literature on the efficiency of directed search with posted prices, which I discuss further in the Related Literature section. I also show that if the firms have all the bargaining power, the equilibrium outcomes of random and directed search in the transferable utility model coincide to the corresponding outcomes of the basic model with non-transferable utility. Essentially, when the firms have all the bargaining power, they can extract the full surplus which reduces the workers to non-strategic objects. Furthermore, if the firms do not have all the bargaining power, then equilibrium outcomes in both random and directed search markets converge to a competitive market outcome as the cost of searching vanishes. The competitive market limit result is in line with Lauermann (2013) who provides necessary and sufficient conditions under which the equilibrium outcomes of search and bargaining models converge to a Walrasian outcome as search frictions disappear.

In Online Appendix A, I consider several extensions to the basic model. One of the main assumptions of the basic model is scarcity: the ratio of objects to agents in the market is less than unity. More generally, I characterize two cutoffs $\bar{\lambda} \geq \underline{\lambda}>1$ (which depend on the search cost and the quality distribution) such that random search dominates directed search 
whenever the ratio of objects to agents is below $\underline{\lambda}$, and directed search dominates random search whenever the ratio is above $\bar{\lambda}$. Intuitively, it is still possible to have more objects than agents in the market (so that the scarcity assumption is violated) and yet have more agents than the objects they find desirable in equilibrium. When the mass of objects is not much larger than the mass of agents, directed search protocol still leads to a significant welfare loss due to congestion externalities. On the other hand, if objects are sufficiently plentiful in the market, congestion become negligible and directed search starts to dominate random search in terms of welfare.

In the basic model, I assume that the agents have homogeneous preferences and that search is frictionless. These two assumptions imply that there are no disadvantages in either search protocol due to exogenously assumed coordination failures. ${ }^{4}$ Consequently, the inefficiencies in the basic model arise purely from the agents' strategic response to the underlying search protocol. The same inefficiencies would be present in a more general model even if the magnitudes of these inefficiencies will depend on the additional structure placed on the more general model. In Online Appendix A, I relax the two assumptions and show that if search is not too frictional, or if the amount of preference heterogeneity is small, social welfare is still higher under random search than directed search.

Finally, I also consider a hybrid search protocol: The search market is segmented into finite sub-markets, and each sub-market provides a coarse signal of quality of the objects it contains. The agents first choose which sub-market to enter (similar to directed search) based on the coarse information they observe. The agents then randomly sample objects from within the chosen sub-market (similar to random search). ${ }^{5}$ I show that welfare is higher under the random search protocol than under a hybrid search protocol.

\section{Related Literature}

There is a large literature in search theory that shows that directed search is more efficient than random search. In the context of exchange economies, Butters (1977) shows that search directed through price-advertising leads to socially efficient outcomes, and Peters (1991) shows that directed search yields a more efficient outcome than random search. Additionally, Peters (1997, 2000) shows that the equilibrium allocations in finite markets with directed search converge

\footnotetext{
${ }^{4}$ For example, most frictional search models assume (implicitly or explicitly) that more agent-object pairs form when agents search within one big market, as in random search, than when agents search in smaller segmented sub-markets, as in directed search. This would confer an exogenously assumed disadvantage on directed search. Conversely, introducing horizontal differentiation adds an advantage to directed search which facilitates assortative matching.

${ }^{5}$ Loosely, directed search is a special case of hybrid search in which the sub-markets provide perfect information while random search is a special case of hybrid search in which the sub-markets provide no information.
} 
to some competitive market outcome as the number of buyers and sellers grows large. In the context of labor markets, Montgomery (1991) and Moen (1997) show that the equilibrium outcomes under directed search correspond to the socially efficient outcomes of a competitive labor market. Similarly, Mortensen and Wright (2002) show that directed search always leads to socially efficient outcomes whereas random search is efficient only when search frictions disappear. The efficiency of directed search also holds in labor search markets with ex-ante firm investments (Acemoglu and Shimer, 1999a) and with risk-averse workers (Acemoglu and Shimer, 1999b), and in markets with two-sided heterogeneity (Shi, 2002; Shimer, 2005; Eeckhout and Kircher, 2010). Even without posted prices, Kim and Kircher (2015) show that directed search based on cheap talk can lead to socially efficient outcomes if the trading mechanism is an efficient first-price auction.

The sharp contrast between the previous literature and the results of this paper stem from the fact that the prior papers study search markets in which there is a mechanism (posted prices or auctions) to internalize the search externalities created by agents resulting in an efficient outcome. In this paper, utility is either non-transferable or is transferable through prices that are determined ex-post. In either case, negative externalities generated in the search markets cannot be fully off-set by changing the ex-post value of a match. Of course, if the agents in this paper could direct their search based on both quality and ex-ante posted prices, there is a socially efficient outcome in this model as well. Hence, one can interpret the results in this paper as showing that in markets without posted prices (or the ability to commit to the posted prices), directed search is not only inefficient but is less efficient than random search.

Menzio $(2005,2007)$ studies a labor search market with ex-post transfers in which firms with differing productivity send cheap talk messages and workers use these messages to choose with which firm to match. Directed search endogenously arises in a separating equilibrium whereas random search arises in a babbling equilibrium. ${ }^{6}$ Similar to this paper, Menzio shows that congestion inefficiencies accompany directed search whenever it arises in equilibrium. However, in a separating equilibrium (which gives rise to directed search), there are also signaling inefficiencies. Furthermore, since the matching technology is concave, fewer matches are created under directed search than under random search. The interaction of all these inefficiencies makes it difficult in Menzio (2005, 2007) to establish an unambiguous relationship between the search protocol and welfare. In contrast, in this paper, there are no signaling distortions as the search protocol is exogenously given. Furthermore, the matching technology is frictionless and places both directed and random search protocols on equal footing. By abstracting away from the

\footnotetext{
${ }^{6}$ In a separating equilibrium, different productivity types send different credible messages.
} 
signaling inefficiencies and frictional matching technology, this paper identifies one of the key sources of welfare loss in directed search protocol.

More generally, we can interpret the differences between random and directed search as a difference in the timing of information. Agents have more ex-ante information about their match payoffs under directed search than under random search. As such, the adverse effects of ex-ante information in search markets studied in this paper are in the same spirit to the Hirshleifer effect in exchange economies (Hirshleifer, 1971; Schlee, 2001). More recently, Lester et al. (2016) show that increasing transparency in a search market for "lemons" is sometimes detrimental.

This paper is also related to the literature on adverse selection in search markets. The effect of adverse selection has been studied by Burdett and Coles (1997) in marriage matching markets, Davis (2001) in labor markets, Inderst (2005) in insurance markets, and Lauermann (2012) in exchange economies. However, all these papers focus only on random search markets.

While not directly related to this paper, the literature on market and search platform design has pointed out that limiting choices (Halaburda et al., 2017), limiting the number of potential matches (Arnosti et al., 2015), limiting preference signaling (Coles et al., 2013), or preventing one side of the market from initiating contact with potential matches (Kanoria and Saban, 2017) could all be welfare improving.

The parsimonious search model in this paper can be seen as a variant of Gale (1987), and Satterthwaite and Shneyerov (2007, 2008). Recently, Lauermann (2013) uses a similar model of search to study how limits of equilibrium allocations relate to competitive market outcomes when search frictions disappears. Similarly, Olszewski and Wolinsky (2016) use a similar model to study search markets for objects with multiple attributes.

The remainder of the paper is structured as follows: In Section 1, I describe the basic model, derive the unique stationary equilibrium outcomes under random and directed search, and present the main result that random search is more efficient than directed search in markets with scarcity. In Section 2, I extend the model to accommodate transfers and derive the equilibrium outcomes in a labor search market with ex-post Nash bargaining. Section 3 concludes. All remaining extensions are presented in the Online Appendix. 


\section{Basic Search Model}

\subsection{Setup}

I consider a search market with agents (she) on one side and objects (it) on the other side. The objects are of differing quality denoted by $\theta \in \Theta \triangleq[0,1]$. I assume that the objects are non-strategic, i.e., they make no decisions and have no preferences. ${ }^{7}$ In contrast, the agents are strategically searching with the hopes of being matched to a high quality object.

Time is discrete. In each period, the agents and the objects in the search market are paired together. The specific details of how they pair-up is discussed later. If an agent has been paired to an object, she observes its quality $\theta$ and either matches with it or rejects it. If the agent decides to match, she exits the market with the object and gets a utility of $u(\theta)=\theta$. If the agent rejects the object, both the object and the agent return to the search market. In each period the agent is unmatched, she gets a payoff of 0 .

Before the period ends, a fraction $\gamma \in(0,1)$ of the agents and objects left in the search market "die". Finally, a mass $k_{a}$ of new agents and a mass $k_{o}$ of new objects flow into the market. The newly arriving objects have quality distributed according to an absolutely continuous distribution function $F$ with a bounded and positive density function $f .{ }^{8}$ An important feature of the search model is scarcity: (weakly) fewer objects than agents flow into the market with $k_{a} \geq k_{o}>0$.

The search market in each period $t$ is characterized by the tuple $\Psi_{t} \triangleq\left\langle G_{t}, M_{t}^{a}, M_{t}^{o}\right\rangle$ where $G_{t}: \Theta \rightarrow[0,1]$ is an endogenously determined market quality distribution, $M_{t}^{a}$ is the total mass of agents in the market, and $M_{t}^{o}$ is the total mass of objects in the market. Thus, $M_{t}^{o} G_{t}(\theta)$ represents the total mass of objects available in the search market in period $t$ whose quality is at most $\theta$. I will refer to $\Psi_{t}$ as the market composition in period $t$. The strategic decisions made by the agents in period $t$ determine the market composition in $t+1$, and so on.

\subsection{Steady State Markets}

Throughout the paper, the focus is on steady state Markov outcomes: an agent's decision in period $t$ depends only on the current market composition $\Psi_{t}$. Furthermore, the market composition is stationary with $\Psi_{t}=\Psi_{t+1}$. Henceforth, I will omit all time indices.

\footnotetext{
${ }^{7}$ In Section 2, I consider a search market with strategic players on both sides: firms (instead of agents) and workers (instead of objects) who differ in their productivity.

${ }^{8}$ Specifically, there exist constants $0<\underline{f}<\bar{f}<\infty$ such that $f(\theta) \in(\underline{f}, \bar{f})$ for all $\theta \in \Theta$.
} 
Let $\ell: \Theta \rightarrow[0,1]$ be a measurable function where $\ell(\theta)$ is the probability that a type $\theta$ object exits the market through a match with an agent under some steady state outcome. Let $\boldsymbol{G}(\cdot ; \ell): \Theta \rightarrow[0,1]$ be the market distribution that arises in such a steady state, and let $\boldsymbol{M}^{a}(\ell)$ and $\boldsymbol{M}^{\circ}(\ell)$ be the corresponding market population of agents and objects respectively.

The steady state market is characterized by the balance of the outflow and the inflow of objects and agents in each period. For each $\theta^{\prime}<\theta^{\prime \prime}$, the inflow-outflow dynamics for objects must satisfy

$$
\underbrace{k_{o}\left(F\left(\theta^{\prime \prime}\right)-F\left(\theta^{\prime}\right)\right)}_{\text {inflow }}=\underbrace{\boldsymbol{M}^{o}(\ell) \int_{\theta^{\prime}}^{\theta^{\prime \prime}}(\ell(\omega)+(1-\ell(\omega)) \gamma) d \boldsymbol{G}(\omega ; \ell)}_{\text {outflow }} .
$$

Similarly, the inflow-outflow dynamics for agents must satisfy

$$
\underbrace{k_{a}}_{\substack{\text { inflow of } \\
\text { agents }}}=\underbrace{\boldsymbol{M}^{o}(\ell) \int_{\Theta} \ell(\omega) d \boldsymbol{G}(\omega ; \ell)}_{\begin{array}{c}
\text { outflow via } \\
\text { match }
\end{array}}+\underbrace{\gamma\left(\boldsymbol{M}^{a}(\ell)-\boldsymbol{M}^{o}(\ell) \int_{\Theta} \ell(\omega) d \boldsymbol{G}(\omega ; \ell)\right)}_{\begin{array}{c}
\text { outflow via } \\
\text { death }
\end{array}} .
$$

Since a match involves an object-agent pair, the mass of agents that leave the market through a match with an object is equal to the mass of objects that leave the market through a match with an agent, given by the first term on the right-hand side of (2). Of the remaining agents, a fraction $\gamma$ leave through death, given by second term on the right-hand side of (2).

\section{Lemma 1}

Let $\ell: \Theta \rightarrow[0,1]$ be some measurable function with $\ell(\theta)$ representing the probability that a type $\theta$ object leaves the market through a match. For any $\gamma>0$, there exists a unique steady state market composition $\Psi=\left\langle\boldsymbol{G}(\cdot ; \ell), \boldsymbol{M}^{a}(\ell), \boldsymbol{M}^{o}(\ell)\right\rangle$ with

$$
\begin{aligned}
& \boldsymbol{G}(\theta ; \ell)=\mathbb{E}_{F}\left[(\gamma+(1-\gamma) \ell)^{-1}\right]^{-1} \int_{0}^{\theta}(\gamma+(1-\gamma) \ell(\omega))^{-1} d F(\omega), \forall \theta \in \Theta \\
& \boldsymbol{M}^{o}(\ell)=k_{o} \mathbb{E}_{F}\left[(\gamma+(1-\gamma) \ell)^{-1}\right] \\
& \boldsymbol{M}^{a}(\ell)=\boldsymbol{M}^{o}(\ell)+\gamma^{-1}\left(k_{a}-k_{o}\right) .
\end{aligned}
$$

Proof: Appendix. 
From (3), it is straightforward to check that that the market distribution $\boldsymbol{G}(\cdot ; \ell)$ is absolutely continuous. Thus, it admits a density function $\boldsymbol{g}(\cdot ; \ell)$ which is almost everywhere positive and bounded. Furthermore, $\boldsymbol{M}^{a}(\ell)$ is greater than $\boldsymbol{M}^{o}(\ell)$ if, and only if, $k_{a}$ is greater than $k_{o}$.

It is important to note that the above characterization takes $\ell$ as exogenously given. Over the next few sections, $\ell$ is endogenously derived in equilibrium from the agents' strategy, which itself depends on the market composition.

\subsection{Random Search Protocol}

Under a random search protocol, the agents and objects in the search market are randomly paired in each period. An agent observes an object's type only after being paired to it. Prior to a pairing, the agent only knows the prevailing market composition $\Psi \triangleq\left\langle G, M^{a}, M^{o}\right\rangle$.

I assume that the maximal number of pairs is achieved. The assumption implies that there are no inefficiencies due to some exogenously assumed coordination failure in the search and matching technology. As there are (weakly) more agents than objects, each object gets paired to a single agent with probability 1 whereas each agent gets paired to a single object with probability $M^{o} / M^{a}$.

An agent who is paired to an object either matches and exits the market or declines to match and searches again next period with probability $1-\gamma$. For a given market composition $\Psi$, let $U^{r}(\Psi)$ be an agent's value from randomly searching, defined by

$$
U^{r}(\Psi)=\frac{M^{o}}{M^{a}} \int_{\Theta} \max \left\{\theta,(1-\gamma) U^{r}(\Psi)\right\} d G(\theta)+\left(1-\frac{M^{o}}{M^{a}}\right)(1-\gamma) U^{r}(\Psi) .
$$

An agent's optimal strategy is given by a cutoff $\bar{\theta}(\Psi)$ such that the agent matches to any object of quality $\theta>\bar{\theta}(\Psi)$ and rejects any object of quality $\theta<\bar{\theta}(\Psi)$. The cutoff is characterized by the indifference condition

$$
\bar{\theta}(\Psi)=(1-\gamma) U^{r}(\Psi)
$$

the marginal quality at which the agent is indifferent between matching to her current object and continuing to search for a possibly better match. ${ }^{9}$

Each agent's cutoff strategy depends on the market composition $\Psi$ which she takes as a given. However, the market composition is itself an endogenous variable that depends on the

\footnotetext{
${ }^{9}$ Since $\Theta=[0,1]$ and $\gamma \in(0,1)$, it is never the case that $\bar{\theta}(\Psi)=0$; the agent can always do better by continuing to search and accepting any quality strictly greater than her outside option. The results in the paper easily generalize to the case where $\Theta=\left[\theta_{l}, \theta_{u}\right]$ where the indifference condition satisfies $\bar{\theta}(\Psi) \geq(1-\gamma) U^{r}(\Psi)$, with equality if $\bar{\theta}(\Psi)>\theta_{l}$.
} 
aggregate effect of the agents' strategies.

When all agents follow a cutoff strategy $\hat{\theta} \in \Theta$, any object of type $\theta<\hat{\theta}$ leaves the market through a match with probability $\ell(\theta)=0$, while any object of type $\theta>\hat{\theta}$ leaves the market through a match with probability $\ell(\theta)=1$. The market composition as described in (3)-(5) then takes a particularly simple form; with some abuse of notation, $\Psi=\left\langle\boldsymbol{G}(\cdot ; \hat{\theta}), \boldsymbol{M}^{a}(\hat{\theta}), \boldsymbol{M}^{o}(\hat{\theta})\right\rangle$ is given by: ${ }^{10}$

$$
\begin{aligned}
& \boldsymbol{G}(\theta ; \hat{\theta})=\left\{\begin{array}{c}
\frac{F(\theta)}{\gamma+(1-\gamma) F(\hat{\theta})} \quad \text { for } \quad \theta \leq \hat{\theta} \\
\frac{\gamma F(\theta)+(1-\gamma) F(\hat{\theta})}{\gamma+(1-\gamma) F(\hat{\theta})} \quad \text { for } \quad \theta \geq \hat{\theta}
\end{array}\right. \\
& \boldsymbol{M}^{o}(\hat{\theta})=\gamma^{-1} k_{o}(\gamma+(1-\gamma) F(\hat{\theta})), \\
& \boldsymbol{M}^{a}(\hat{\theta})=\gamma^{-1}\left(k_{a}-k_{o}(1-\gamma)(1-F(\hat{\theta}))\right) .
\end{aligned}
$$

Definition 1 A random search stationary Markov Equilibrium (SME) is given by a cutoff $\theta_{r} \in \Theta$ and a market composition $\Psi_{r}$ such that

(i) $\theta_{r}=\bar{\theta}\left(\Psi_{r}\right)$, and

(ii) $\Psi_{r}=\left\langle\boldsymbol{G}\left(\cdot ; \theta_{r}\right), \boldsymbol{M}^{a}\left(\theta_{r}\right), \boldsymbol{M}^{o}\left(\theta_{r}\right)\right\rangle$ as defined by (6)-(8).

In other words, the pair $\left(\theta_{r}, \Psi_{r}\right)$ constitute an equilibrium if $(i)$ given the market composition $\Psi_{r}$, it is sequentially rational for each agent to only match with objects whose quality is above $\theta_{r}$, and (ii) the market composition $\Psi_{r}$ is consistent with a steady state outcome as given by (6)-(8) when all agents use the cutoff $\theta_{r}$.

\section{Proposition 1}

There is a unique random search SME given by $\left(\theta_{r}^{*}, \Psi_{r}^{*}\right)$. The cutoff $\theta_{r}^{*}$ satisfies the indifference condition

$$
\theta_{r}^{*}=(1-\gamma) \frac{k_{o}}{k_{a}} \int_{\theta_{r}^{*}}^{1} \omega d F(\omega)
$$

Proof. Fix some market composition $\Psi \triangleq\left\langle G, M^{a}, M^{o}\right\rangle$. An agent's optimal cutoff is $\bar{\theta}(\Psi)$. $\overline{{ }^{10} \text { Specifically, } \boldsymbol{G}(\cdot ; \hat{\theta})=\boldsymbol{G}(\cdot ; \ell) \text { with } \ell(\theta)=0}$ if $\theta<\hat{\theta}$ and $\ell(\theta)=1$ if $\theta>\hat{\theta}$. 
Hence, the value from random search is given by

$$
\begin{aligned}
U^{r}(\Psi) & =\frac{M^{o}}{M^{a}} \int_{\bar{\theta}(\Psi)}^{1} \omega d G(\omega)+(1-\gamma) U^{r}(\Psi)\left(\frac{M^{o}}{M^{a}} G(\bar{\theta}(\Psi))+1-\frac{M^{o}}{M^{a}}\right) \\
& =\frac{\frac{M^{o}}{M^{a}} \int_{\bar{\theta}(\Psi)}^{1} \omega d G(\omega)}{\gamma+\frac{M^{o}}{M^{a}}(1-\gamma)(1-G(\bar{\theta}(\Psi)))} .
\end{aligned}
$$

The pair $\left(\theta_{r}, \Psi_{r}\right)$ constitutes an SME if $\theta_{r}=\bar{\theta}\left(\Psi_{r}\right)$ and $\Psi_{r}=\left\langle\boldsymbol{G}\left(\cdot ; \theta_{r}\right), \boldsymbol{M}^{a}\left(\theta_{r}\right), \boldsymbol{M}^{o}\left(\theta_{r}\right)\right\rangle$. Substituting in the market composition expressions in (6)-(8) simplifies the value function to

$$
U^{r}\left(\Psi_{r}\right)=\frac{k_{o}}{k_{a}} \int_{\theta_{r}}^{1} \omega d F(\omega) .
$$

Recall that an agent's cutoff strategy for a given market composition $\Psi$ is characterized by the indifference condition $\bar{\theta}(\Psi)=(1-\gamma) U^{r}(\Psi)$. Thus, $\theta_{r}=\bar{\theta}\left(\Psi_{r}\right)$ if, and only if,

$$
\theta_{r}=(1-\gamma) \frac{k_{o}}{k_{a}} \int_{\theta_{r}}^{1} \omega d F(\omega)
$$

Define $\mathbb{U}: \Theta \rightarrow \Theta$ by $\mathbb{U}(\theta)=\int_{\theta}^{1} \omega d F(\omega)$ which is a continuous and strictly decreasing function. Hence, there is a unique fixed point to the function $(1-\gamma) \frac{k_{o}}{k_{a}} \mathbb{U}$ given by $\theta_{r}^{*}$. The market composition $\Psi_{r}^{*}$ is then derived from the cutoff $\theta_{r}^{*}$ using (6)-(8)

The characterization of the random search SME cutoff $\theta_{r}^{*}$ is similar to the characterization of a reservation wage in the McCall search model (McCall, 1970) except the market composition in the current model is endogenously derived. Intuitively, an agent who searches randomly faces an inter-temporal trade off; she can either settle for the object to which she is currently paired or she can continue randomly searching for an object with a higher quality. However, an agent who declines to match today can continue to search only if she survives into the next period. Thus, her continuation value of search must be scaled down by her "effective discount factor" $1-\gamma$. The indifference condition (9) captures the resolution of this inter-temporal trade off. The cutoff $\theta_{r}^{*}$, which I refer to as pickiness, is the lowest quality an agent is willing to settle for today after accounting for her discounted net present value of searching in the future, given by $(1-\gamma) \frac{k_{o}}{k_{a}} \int_{\theta_{r}^{*}}^{1} \omega d F(\omega)$.

For a given $\gamma>0$, let $\theta_{r}^{*}(\gamma)$ be the unique equilibrium cutoff satisfying the indifference 
condition (9). Let $\boldsymbol{U}_{\gamma}^{r}=\frac{\theta_{r}^{*}(\gamma)}{1-\gamma}$ be the value of random search in the unique SME.

As $\gamma$ decreases, the effective discount factor increases which in turn increases the discounted value of search. Thus, agents becomes less willing to settle for low quality objects, i.e., they become pickier. However, in the aggregate, the pickier the agents are, the more negative externality they impose on the market. Thus, as $\gamma$ decreases, the undiscounted value of search decreases.

\section{Corollary 1}

(i) $\theta_{r}^{*}(\gamma)$ is decreasing in $\gamma$, i.e., the agent becomes less picky as $\gamma$ increases, and

(ii) $\boldsymbol{U}_{\gamma}^{r}$ is increasing in $\gamma$, i.e., the agent's value of search increases as $\gamma$ increases.

The unique equilibrium outcome in a random search market is inefficient. The efficient outcome would be for all agents to match with the first object they are paired to which would clear the market as quickly as possible (taking into account that there are weakly more agents than objects) and would yield a higher average payoff of $\frac{k_{o}}{k_{a}} \mathbb{E}_{F}[\theta]$. However, matching to an object regardless of quality is not sequentially rational for any individual agent. In equilibrium, an agent instead chooses to match to all types above $\theta_{r}^{*}(\gamma)$ after taking into account the negative externalities that other agents impose on the market composition. However, agents do not fully internalize the negative externalities of their own strategic choices. Hence, the random search SME is inefficient.

A key drawback of a random search protocol is that agents are forced to pair with positive probability to objects they would rather avoid. In the following section, I consider a directed search protocol in which the agents have more ex-ante information that they can use to only pair with the objects they deem desirable.

\subsection{Directed Search Protocol}

In a directed search protocol, the search market is further subdivided into a continuum of sub-markets. A sub-market $S(\theta)$ exclusively contains all objects in the market with quality $\theta$. Given a market composition $\Psi \triangleq\left\langle G, M^{a}, M^{o}\right\rangle$, sub-market $S(\theta)$ has a "mass" of $M^{o} g(\theta)$ objects, where $g$ is the density function of $G$.

Agents can distinguish between all the sub-markets and can choose which sub-market to search in for a given period. Therefore, each agent knows not only the market composition but also the quality of an object prior to pairing with it. Let $Q(\theta)$ be the fraction of agents 
searching in sub-markets $\cup_{\theta^{\prime} \leq \theta} S\left(\theta^{\prime}\right)$. I will refer to $Q$ as the queueing $C D F$, even though there is no actual queue in the formal sense of the word. I assume that $Q$ is absolutely continuous with respect to the steady state market distribution $G$, i.e., agents cannot enter into a sub-market that does not exist. Since $G$ is absolutely continuous with respect to $F$ (Lemma 1 ) which is itself absolutely continuous, $Q$ admits a density function $q$. I will refer to $M^{a} q(\theta)$ as the queue length in sub-market $S(\theta)$.

In each period, agents choose which sub-markets to enter. The agents and objects within a sub-market are paired randomly so that the maximal number of pairs is achieved. The long-side of the sub-market is rationed while the short-side is guaranteed a pairing. Let $\alpha(\theta ; Q, \Psi)=$ $\min \left\{\frac{M^{o} g(\theta)}{M^{a} q(\theta)}, 1\right\}$ be the probability that an agent in sub-market $S(\theta)$ gets paired to an object in a given period. Similarly, let $\beta(\theta ; Q, \Psi)=\min \left\{\frac{M^{a} q(\theta)}{M^{\circ} g(\theta)}, 1\right\}$ be the probability that an object in sub-market $S(\theta)$ gets paired to an agent in a given period.

To simplify exposition, I assume that an agent matches with the first object to which she is paired. The assumption is without loss of generality as it is never rational for an agent to enter a sub-market and then reject an object to which she is paired. After all, the agent cannot get paired to a higher quality object by waiting in the same sub-market and could have done better by choosing a different sub-market to enter in the first place. As such, I will refer to $\alpha(\theta ; Q, \Psi)$ as the agent match probability and $\beta(\theta ; Q, \Psi)$ as the object match probability.

Additionally, I assume without loss of generality that agents remain in the first sub-market they enter. In equilibrium, agents must be indifferent among all of the sub-markets they enter. Whether or not an agent remains in the first sub-market she enters is irrelevant for her payoff.

Given a queuing $\operatorname{CDF} Q$ and a market composition $\Psi$, let $U^{d}(\theta ; Q, \Psi)$ be the agent's value from entering sub-market $S(\theta)$, given by

$$
\begin{gathered}
U^{d}(\theta ; Q, \Psi)=\alpha(\theta ; Q, \Psi) \theta+(1-\gamma)(1-\alpha(\theta ; Q, \Psi)) U^{d}(\theta ; Q, \Psi) \\
=\frac{\alpha(\theta ; Q, \Psi) \theta}{\gamma+\alpha(\theta ; Q, \Psi)(1-\gamma)}
\end{gathered}
$$

An agent's decision of which sub-market to enter is affected by $Q$ and $\Psi$ which she takes as a given. However, both $Q$ and $\Psi$ themselves are affected by the agents' strategies.

Definition 2 A directed search $S M E$ is given by a pair $\left(Q_{d}, \Psi_{d}\right)$ such that

(i) $Q_{d}(\theta)=\int_{0}^{\theta} q_{d}(\omega) d \omega$ for all $\theta \in \Theta$ with $Q_{d}(1)=1$,

(ii) $q_{d}(\theta)>0$ implies $\theta \in \arg \max _{\theta^{\prime} \in \Theta} U^{d}\left(\theta^{\prime} ; Q_{d}, \Psi_{d}\right)$, and 
(iii) $\Psi_{d}=\left\langle\boldsymbol{G}\left(\cdot ; \beta\left(\cdot ; Q_{d}, \Psi_{d}\right)\right), \boldsymbol{M}^{a}\left(\beta\left(\cdot ; Q_{d}, \Psi_{d}\right)\right), \boldsymbol{M}^{o}\left(\beta\left(\cdot ; Q_{d}, \Psi_{d}\right)\right)\right\rangle$ as given by $(3)-(5)$.

In other words, the pair $\left(Q_{d}, \Psi_{d}\right)$ constitute an equilibrium if $(i) Q_{d}$ is an absolutely continuous queuing CDF, (ii) given agent match probabilities $\alpha\left(\cdot ; Q_{d}, \Psi_{d}\right)$, agents enter only the sub-markets that maximize their payoff, and (iii) given object match probabilities $\beta\left(\cdot ; Q_{d}, \Psi_{d}\right)$, the market composition is characterized by (3)-(5). ${ }^{11}$

In order to characterize the equilibrium, I make the following observation that simplifies the problem.

\section{Lemma 2}

If $\left(Q_{d}, \Psi_{d}\right)$ is a directed search SME, there exists a cutoff $\theta_{d} \in \operatorname{Int}(\Theta) \triangleq(0,1)$ such that

(i) $q_{d}(\theta)=0$ if $\theta<\theta_{d}$ and $q_{d}(\theta)>0$ if $\theta>\theta_{d}$,

(ii) $U^{d}\left(\theta ; Q_{d}, \Psi_{d}\right)=\left\{\begin{array}{c}\theta \text { for } \theta \leq \theta_{d} \\ \theta_{d} \text { for } \theta \geq \theta_{d}\end{array}\right.$, and

(iii) $\Psi_{d}=\left\langle\boldsymbol{G}\left(\cdot ; \theta_{d}\right), \boldsymbol{M}^{a}\left(\theta_{d}\right), \boldsymbol{M}^{o}\left(\theta_{d}\right)\right\rangle$ as given by $(6)-(8)$.

\section{Proof.}

Let $\left(Q_{d}, \Psi_{d}\right)$ be a directed search SME. By definition, $q_{d}(\theta)>0$ only if $\theta \in \arg \max _{\theta^{\prime}} U^{d}\left(\theta^{\prime} ; Q_{d}, \Psi_{d}\right)$. Assume $q_{d}\left(\theta^{\prime}\right)>0=q_{d}\left(\theta^{\prime \prime}\right)$ for some $\theta^{\prime}<\theta^{\prime \prime}$. Then, $\alpha\left(\theta^{\prime \prime} ; Q_{d}, \Psi_{d}\right)=\min \left\{\frac{M^{o} g_{d}\left(\theta^{\prime \prime}\right)}{M^{a} q_{d}\left(\theta^{\prime \prime}\right)}, 1\right\}=1$ and $U^{d}\left(\theta^{\prime \prime} ; Q_{d}, \Psi_{d}\right)=\theta^{\prime \prime}>\theta^{\prime} \geq U^{d}\left(\theta^{\prime} ; Q_{d}, \Psi_{d}\right)$, which is a contradiction.

Therefore, there is a cutoff $\theta_{d}$ such that agents only enter sub-markets above $\theta_{d}$. If $\theta_{d}=1$, then the queueing $\mathrm{CDF}$ would be $Q_{d}(\theta)=\mathbb{1}_{[\theta=1]}$, violating the absolute continuity of $Q_{d}$. Hence, $\theta_{d}<1$ and $q_{d}(\theta)>0$ for all $\theta>\theta_{d}$.

The sub-markets above $\theta_{d}$ must be payoff-maximizing: $U^{d}\left(\theta ; Q_{d}, \Psi_{d}\right) \geq U^{d}\left(\theta_{d} ; Q_{d}, \Psi_{d}\right)$ for all $\theta>\theta_{d}$. However, $0 \leq U^{d}\left(\theta ; Q_{d}, \Psi_{d}\right) \leq \theta$ for all $\theta \in \Theta$, which implies

$$
0 \leq U^{d}\left(\theta_{d} ; Q_{d}, \Psi_{d}\right) \leq \lim _{\theta \downarrow \theta_{d}} U^{d}\left(\theta ; Q_{d}, \Psi_{d}\right) \leq \theta_{d}
$$

\footnotetext{
${ }^{11}$ Recall it is WLOG to assume that an agent matches with any object she pairs with in equilibrium. Hence $\ell(\theta)=\beta\left(\theta ; Q_{d}, \Psi_{d}\right)$.
} 
Moreover, as the agents must be indifferent to all the sub-markets they enter, $U^{d}\left(\theta^{\prime \prime} ; Q_{d}, \Psi_{d}\right)=$ $U^{d}\left(\theta^{\prime} ; Q_{d}, \Psi_{d}\right)=U^{d}\left(\theta_{d} ; Q_{d}, \Psi_{d}\right)$ for all $\theta^{\prime \prime}>\theta^{\prime}>\theta_{d}{ }^{12}$

If $\theta_{d}=0$, then for all $\theta \in \Theta, U^{d}\left(\theta ; Q_{d}, \Psi_{d}\right)=0$ which would require $\alpha\left(\theta ; Q_{d}, \Psi_{d}\right)=0$. However, this is only possible if $q_{d}(\theta)$ is unbounded for all $\theta \in \Theta$, once again violating the absolute continuity of $Q_{d}$. Hence, $\theta_{d}>0$ and $q_{d}(\theta)=0$ for all $\theta<\theta_{d}$.

Furthermore, for all $\theta<\theta_{d}$, the agent match probability $\alpha\left(\theta ; Q_{d}, \Psi_{d}\right)=1$ as the queue length is zero below the cutoff. Hence, $U^{d}\left(\theta ; Q_{d}, \Psi_{d}\right)=\theta$ for all $\theta<\theta_{d}$, which implies $\lim _{\theta \uparrow \theta_{d}} U^{d}\left(\theta ; Q_{d}, \Psi_{d}\right)=\theta_{d}$. Combining the left and right limits yields $U^{d}\left(\theta_{d} ; Q_{d}, \Psi_{d}\right)=\theta_{d}$.

To sustain indifference across all the sub-markets above $\theta_{d}$, it is necessary that $\alpha\left(\theta ; Q_{d}, \Psi_{d}\right)<1$ for all $\theta>\theta_{d}$. Therefore, $\beta\left(\theta ; Q_{d}, \Psi_{d}\right)=1$ for all $\theta>\theta_{d}$. In other words, all objects in submarkets $S(\theta)$ with $\theta>\theta_{d}$ exit the search market only through a match.

Furthermore, $q_{d}(\theta)=0$ for all $\theta<\theta_{d}$ implies that $\beta\left(\theta ; Q_{d}, \Psi_{d}\right)=\min \left\{\frac{M^{a} q_{d}(\theta)}{M^{\circ} g_{d}(\theta)}, 1\right\}=0$. Thus, objects in sub-markets $S(\theta)$ with $\theta<\theta_{d}$ exit only through death. We can now express the market composition using (6)-(8) with the cutoff $\hat{\theta}=\theta_{d}$.

If agents enter a sub-market $S\left(\theta^{\prime}\right)$ in equilibrium, then they must enter all sub-markets containing objects of quality $\theta>\theta^{\prime}$. Otherwise, if the queue length at $S\left(\theta^{\prime \prime}\right)$ for some $\theta^{\prime \prime}>\theta^{\prime}$ were 0 , an agent could profitably deviate by entering $S\left(\theta^{\prime \prime}\right)$ and matching to an object of a higher quality with probability 1.

Yet, agents must be indifferent among all the sub-markets they enter in equilibrium. Consequently, sub-markets that contain objects of higher quality must also have longer queue lengths (lower agent match probabilities). In other words, for almost all sub-markets the agents enter, the agents must be on the long-side while the objects are on the short-side.

Combining these two observations, there exists some cutoff $\theta_{d}$ such that objects with quality above $\theta_{d}$ leave the market only through a match while objects with quality below $\theta_{d}$ leave the market only through death. The inflow and outflow of objects now closely resemble the dynamics in the random search model, yielding a similar market composition.

\footnotetext{
${ }^{12}$ The equality follows because if $U^{d}\left(\theta_{d} ; Q_{d}, \Psi_{d}\right)<\lim _{\theta \downarrow \theta_{d}} U^{d}\left(\theta ; Q_{d}, \Psi_{d}\right) \leq \theta_{d}$, then agents would strictly prefer to not enter sub-market $S\left(\theta_{d}\right)$. However, this would mean $\alpha\left(\theta_{d} ; Q_{d}, \Psi_{d}\right)=1$ as the queue length would be zero, which in turn implies $U^{d}\left(\theta_{d} ; Q_{d}, \Psi_{d}\right)=\theta_{d}$.
} 
The following proposition characterizes the direct search SME and shows that it is uniquely pinned down by the cutoff $\theta_{d}$.

\section{Proposition 2}

There is a unique directed search SME given by $\left(Q_{d}^{*}, \Psi_{d}^{*}\right)$ where

(i) $\Psi_{d}^{*}=\left\langle\boldsymbol{G}\left(\cdot ; \theta_{d}^{*}\right), \boldsymbol{M}^{a}\left(\theta_{d}^{*}\right), \boldsymbol{M}^{o}\left(\theta_{d}^{*}\right)\right\rangle$,

(ii) $Q_{d}^{*}(\theta)=\left\{\begin{array}{cc}0 & \text { if } \theta \leq \theta_{d}^{*} \\ \int_{\theta_{d}^{*}}^{\theta} \frac{k_{o}\left(\omega-(1-\gamma) \theta_{d}^{*}\right)}{\left(k_{a}-k_{o}(1-\gamma)\left(1-F\left(\theta_{d}^{*}\right)\right)\right) \theta_{d}^{*}} d F(\omega) & \text { for } \theta \geq \theta_{d}^{*}\end{array}\right.$.

The cutoff $\theta_{d}^{*} \in \Theta$ satisfies the indifference condition

$$
\theta_{d}^{*}=\frac{k_{o}}{k_{a}} \int_{\theta_{d}^{*}}^{1} \omega d F(\omega)
$$

Proof. Let $\left(Q_{d}, \Psi_{d}\right)$ be a directed search SME with $\Psi_{d} \triangleq\left\langle G_{d}, M_{d}^{a}, M_{d}^{o}\right\rangle$. From Lemma 2, there exists a cutoff $\theta_{d} \in \Theta$ such that $q_{d}(\theta)>(=) 0$ for all $\theta>(<) \theta_{d}$, and $U^{d}\left(\theta ; Q_{d}, \Psi_{d}\right)=\theta_{d}$ for all $\theta \geq \theta_{d}$.

For all $\theta>\theta_{d}, U^{d}\left(\theta ; Q_{d}, \Psi_{d}\right)=\theta_{d}$ necessitates that $\alpha\left(\theta ; Q_{d}, \Psi_{d}\right)=\frac{\gamma \theta_{d}}{\theta-(1-\gamma) \theta_{d}}$. Additionally, because agents must be on the long-side of each sub-market above $\theta_{d}$, we also have that $\alpha\left(\theta ; Q_{d}, \Psi_{d}\right)=\min \left\{\frac{g_{d}(\theta)}{q_{d}(\theta)} \zeta_{d}, 1\right\}=\frac{g_{d}(\theta)}{q_{d}(\theta)} \zeta_{d}$ where $\zeta_{d}=\frac{M_{d}^{o}}{M_{d}^{\alpha}}$. Equating these two expressions for the agent match probability and integrating over the interval $\left[\theta_{d}, 1\right]$ gives

$$
\gamma \theta_{d} \int_{\theta_{d}}^{1} q_{d}(\omega) d \omega=\zeta_{d} \int_{\theta_{d}}^{1} \omega d G_{d}(\omega)-\zeta_{d}(1-\gamma) \theta_{d}\left(1-G_{d}\left(\theta_{d}\right)\right)
$$

Since $q_{d}(\theta)=0$ for all $\theta<\theta_{d}, \int_{\theta_{d}}^{1} q_{d}(\omega) d \omega=\int_{\Theta} q_{d}(\omega) d \omega=1$ (because $Q$ is a CDF). Hence, the expression simplifies to

$$
\theta_{d}=\frac{\zeta_{d} \int_{\theta_{d}}^{1} \theta d G_{d}(\theta)}{\gamma+\zeta_{d}(1-\gamma)\left(1-G_{d}\left(\theta_{d}\right)\right)}
$$

From Lemma 2, the market composition $\Psi_{d}$ is consistent with $\left\langle\boldsymbol{G}\left(\cdot ; \theta_{d}\right), \boldsymbol{M}^{a}\left(\theta_{d}\right), \boldsymbol{M}^{o}\left(\theta_{d}\right)\right\rangle$ as 
given by (6)-(8). Substituting in these expressions for $G_{d}, M_{d}^{a}$, and $M_{d}^{o}$ gives

$$
\theta_{d}=\frac{k_{o}}{k_{a}} \int_{\theta_{d}}^{1} \omega d F(\omega)
$$

Recall, the function $\mathbb{U}: \Theta \rightarrow \Theta$ defined by $\mathbb{U}(\theta)=\int_{\theta}^{1} \omega d F(\omega)$ is continuous and strictly decreasing. Hence, there is a unique fixed point to $\frac{k_{o}}{k_{a}} \mathbb{U}$ given by $\theta_{d}^{*}$. The market composition $\Psi_{d}^{*}$ is then pinned down from the cutoff $\theta_{d}^{*}$ using (6)-(8). Similarly, the queuing CDF is pinned down from the agent match probability with

$$
\alpha\left(\theta ; Q_{d}^{*}, \Psi_{d}^{*}\right)=\frac{\boldsymbol{M}^{o}\left(\theta_{d}^{*}\right) \boldsymbol{g}\left(\theta ; \theta_{d}^{*}\right)}{\boldsymbol{M}^{a}\left(\theta_{d}^{*}\right) q_{d}^{*}(\theta)}=\frac{\gamma \theta_{d}^{*}}{\theta-(1-\gamma) \theta_{d}^{*}}
$$

for all $\theta>\theta_{d}^{*}$.

Intuitively, an agent in a directed search market faces similar trade-offs present in the random search market, i.e., settle for a low quality object versus search for a high quality object. However, the trade-off under directed search is contemporaneous not inter-temporal. In particular, the agent can either settle for a low quality object she can get with certainty today, or she can queue in a congested market today for the possibility of getting a high quality object. The indifference condition (10) captures the resolution of this contemporaneous trade off. The cutoff $\theta_{d}^{*}$ is the highest quality the agent can get with certainty while $\frac{k_{o}}{k_{a}} \int_{\theta_{d}^{*}}^{1} \omega d F(\omega)$ is her value of directed search from using $Q$ as her searching strategy.

For a slightly different intuition, let us re-write (10) as

$$
k_{a} \theta_{d}^{*}=k_{o} \int_{\theta_{d}^{*}}^{1} \theta d F(\theta) .
$$

The left-hand side is the quality "demanded" by the new agents flowing into the market. Specifically, there are $k_{a}$ new agents that arrive in each period, and each one seeks to get an expected payoff of $\theta_{d}^{*}$. The right-hand side is the total quality "supplied" to the agents. In each period, high quality objects in sub-markets $\cup_{\theta>\theta_{d}^{*}} S(\theta)$ are depleted through matches. Hence, the equality between the left and right-hand side captures the idea that we can deliver a total expected utility of $k_{a} \theta_{d}^{*}$ to the agents only through the newly arriving objects that replenish the sub-markets $\cup_{\theta>\theta_{d}^{*}} S(\theta)$.

For a given $\gamma>0$, let $\theta_{d}^{*}(\gamma)$ be the unique equilibrium cutoff satisfying (10). Let $\boldsymbol{U}_{\gamma}^{d}=\theta_{d}^{*}(\gamma)$ be the agent's unique equilibrium value of directed search. 
Interestingly, the cutoff $\theta_{d}^{*}(\gamma)$ (and thus, the value of directed search) is independent of the cost of searching. As $\gamma$ decreases, an agent who failed to get a match today is more likely to survive into the next period. Hence, she becomes more willing to queue in congested sub-markets that contain high quality objects. However, the same is true of all agents; as $\gamma$ decreases, the mass of agents in the market increases which makes the congestion in the desirable sub-markets worse. Furthermore, the supply of objects in the desirable sub-markets $\cup_{\theta>\theta_{d}^{*}(\gamma)} S(\theta)$ remains unaffected by a change in $\gamma$ as all of these objects leave the market only through a match. In other words, while the cost of searching has decreased, the probability of being rationed a high quality object has also decreased by the same factor. Hence, a decrease in $\gamma$ has no effect in a directed search market. ${ }^{13}$

Corollary 2 The cutoff $\theta_{d}^{*}(\gamma)$ and the value of directed search $\boldsymbol{U}_{\gamma}^{d}$ are constant in $\gamma$.

Similar to the random search protocol, the unique equilibrium outcome under a directed search protocol is inefficient. Agents could have queued in all the sub-markets and matched with any quality, clearing the market as much as possible and delivering an average payoff of $\frac{k_{o}}{k_{a}} \mathbb{E}_{F}[\theta]$. However, an agent queued in a sub-market with low quality objects would have an incentive to deviate and queue in a sub-market with a high quality objects. In equilibrium, an agent instead chooses to queue at sub-markets $\cup_{\theta>\theta_{d}^{*}(\gamma)} S(\theta)$ after taking into account the congestion that other agents create. However, agents do not fully internalize the congestion externalities of their own strategic choices which leads to an inefficient outcome.

In the next section, I take a closer look at the differences between random and directed search protocol in terms of equilibrium payoffs and welfare.

\subsection{Social Welfare Comparison}

In the previous sections, I have noted that the equilibrium outcomes of random and directed search protocols are inefficient. In this section, I show that the inefficiencies created by directed search are worse than the inefficiencies arising from random search.

In a steady state market with long-lived agents who enter and exit the market in each period, the appropriate way to measure welfare is by aggregating the equilibrium payoffs of the

\footnotetext{
${ }^{13}$ Under random search, there were two additional effects: First, the mass of low quality objects increases as more of them survive in the search market. Hence, the likelihood of randomly pairing to these low quality objects increases as $\gamma$ decreases which lowers $\boldsymbol{U}_{\gamma}^{r}$. In directed search, the mass of low quality objects also increases but this has no effect as the agents can choose to not enter the sub-markets containing the low quality objects. Second, under random search, the effective discount factor $(1-\gamma)$ increases making the inter-temporal trade-off cheaper for an agent, which in turn made her more picky. In contrast, the trade off under directed search is contemporaneous and thus does not involve the effective discount factor.
} 
new agents entering the market. ${ }^{14}$ Fix the search cost at $\gamma>0$. Since all agents have the same preferences, social welfare under a random search protocol is given by $\boldsymbol{W}_{\gamma}^{r}=k_{a} \boldsymbol{U}_{\gamma}^{r}$. Similarly, social welfare under a directed search protocol is given by $\boldsymbol{W}_{\gamma}^{d}=k_{a} \boldsymbol{U}_{\gamma}^{d}$.

\section{Theorem 1}

For any $\gamma>0, \boldsymbol{W}_{\gamma}^{r}>\boldsymbol{W}_{\gamma}^{d}$. Furthermore, $\lim _{\gamma \rightarrow 0} \boldsymbol{W}_{\gamma}^{r}-\boldsymbol{W}_{\gamma}^{d}=0$.

Proof. Recall the strictly decreasing function $\mathbb{U}: \Theta \rightarrow \Theta$ defined by $\mathbb{U}(\theta)=\int_{\theta}^{1} \omega d F(\omega)$. The cutoff $\theta_{r}^{*}(\gamma)$ is the unique fixed point of $(1-\gamma) \frac{k_{o}}{k_{a}} \mathbb{U}$ and $\theta_{d}^{*}$ is the unique fixed point of $\frac{k_{o}}{k_{a}} \mathbb{U}$. Thus, for any $\gamma>0, \theta_{r}^{*}(\gamma)<\theta_{d}^{*}(\gamma)$, and

$$
\boldsymbol{W}_{\gamma}^{r}=k_{a} \frac{\theta_{r}^{*}(\gamma)}{1-\gamma} \underbrace{=}_{\substack{\text { by } \\ \text { Proposition } 1}} k_{o} \mathbb{U}\left(\theta_{r}^{*}(\gamma)\right)>k_{o} \mathbb{U}\left(\theta_{d}^{*}(\gamma)\right) \underbrace{=}_{\substack{\text { by } \\ \text { Proposition } 2}} k_{a} \theta_{d}^{*}(\gamma)=\boldsymbol{W}_{\gamma}^{d} \text {. }
$$

Furthermore, as $\gamma$ goes to 0 , the respective unique fixed points of $(1-\gamma) \frac{k_{o}}{k_{a}} \mathbb{U}$ and $\frac{k_{o}}{k_{a}} \mathbb{U}$ converge, i.e. $\lim _{\gamma \rightarrow 0} \theta_{r}^{*}(\gamma)-\theta_{d}^{*}(\gamma)=0$. Thus, $\lim _{\gamma \rightarrow 0} \boldsymbol{W}_{\gamma}^{r}-\boldsymbol{W}_{\gamma}^{d}=0$.

Figure 1 depicts the characterization of cutoffs and equilibrium expected payoffs under random and directed search.

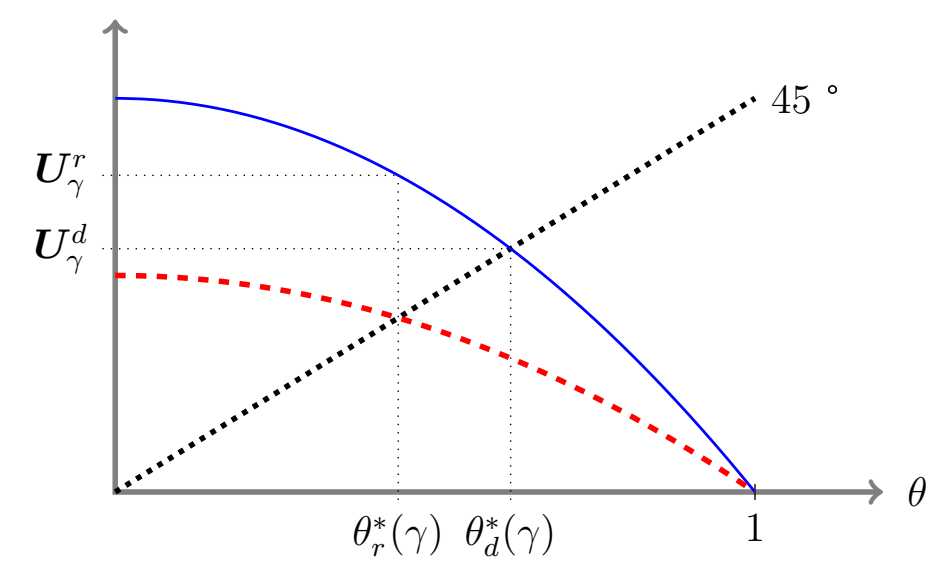

Figure 1: The solid blue curve plots $\frac{k_{o}}{k_{a}} \mathbb{U}$ while the dash-dotted red curve plots $(1-\gamma) \frac{k_{o}}{k_{a}} \mathbb{U}$.

The congestion externalities generated in directed search markets are seemingly different from the adverse selection externalities generated by random search. For instance, when search ${ }^{14}$ See Olszewski and Wolinsky (2016) or Burdett and Menzio (2017) for a discussion. 
is directed, whether or not the market composition over-represents low quality objects does not matter because the agents can simply avoid pairing with these low quality objects.

In order to better understand the relationship between the two protocols and the inefficiencies they generate, consider the following hypothetical search market: agents are paired randomly to objects and forced to match if, and only if, quality is above $\hat{\theta} \in \Theta$. To simplify, let us assume that $k_{a}=k_{o}$ which, by Lemma 1, implies that the mass of agents and objects in the search market are equal. Hence, each agent is guaranteed a pairing with an object of some quality. Let $\hat{\Psi}=\left\langle\boldsymbol{G}(\cdot ; \hat{\theta}), \boldsymbol{M}^{a}(\hat{\theta}), \boldsymbol{M}^{\circ}(\hat{\theta})\right\rangle$ be the resulting market composition as given in $(6)-(8)$. Let $\mathbb{U}(\hat{\theta})$ be an agent's stationary payoff in this hypothetical market given by

$$
\begin{aligned}
\mathbb{U}(\hat{\theta}) & =\int_{\hat{\theta}}^{1} \omega d \boldsymbol{G}(\omega ; \hat{\theta})+(1-\gamma) \mathbb{U}(\hat{\theta}) \boldsymbol{G}(\hat{\theta} ; \hat{\theta}) \\
& =\int_{\hat{\theta}}^{1} \omega d F(\omega),
\end{aligned}
$$

where the last equality follows from substituting the expression for $\boldsymbol{G}(\cdot ; \hat{\theta})$ given by (6).

The pickier the agents are forced to be, the lower their payoff in this hypothetical world. Intuitively, as the agents are forced to become pickier, the fraction of agents who exit through a match decreases because the probability of pairing with an object above the cutoff decreases. To make matters worse, the pickier the agents are, the more adversely selected the market becomes, making it even less likely than an agent pairs with an object above the cutoff. Formally, as $\hat{\theta}$ increases, $\boldsymbol{G}(\hat{\theta} ; \hat{\theta})$ also increases (market becomes more adversely selected); this is not immediately obvious because $G(\cdot ; \hat{\theta})$ is non-monotone in $\hat{\theta}$ as shown in Figure 2. Furthermore, as $\hat{\theta}$ increases, the fraction of agents leaving through a match, $1-\boldsymbol{G}(\hat{\theta} ; \hat{\theta})$, decreases.

Since $\mathbb{U}(\hat{\theta})$ is a decreasing function of $\hat{\theta}$, a social planner would set $\hat{\theta}=0$. However, when the agents can freely choose their pickiness, the equilibrium outcome is $\hat{\theta}=\theta_{r}^{*}(\gamma)>0$ and $\boldsymbol{U}_{\gamma}^{r}=\mathbb{U}\left(\theta_{r}^{*}(\gamma)\right)$. The cutoff $\theta_{r}^{*}(\gamma)$ is an agent's sequentially rational level of pickiness: it is the lowest quality she is willing to settle for today in order to forsake potentially better matches in the future after taking into account the equilibrium market composition. The inefficiencies in a random search market arise precisely because the agents are too picky.

Let us now consider directed search, which we can think of as random search except the likelihood that an agent is paired to an object of a specific quality is not uniformly random over 


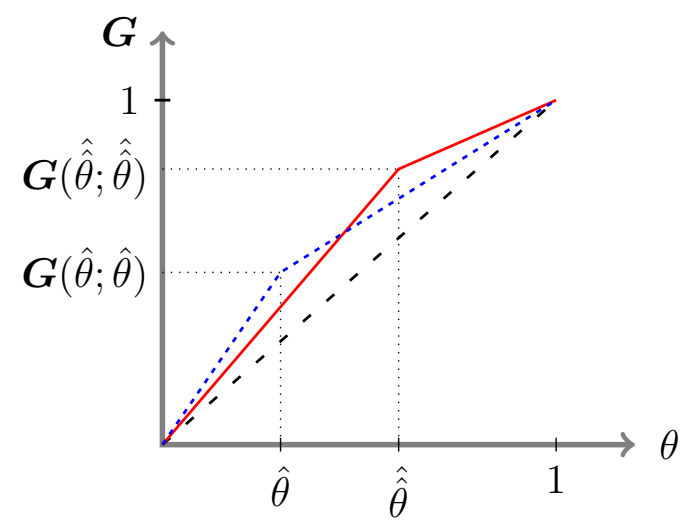

Figure 2: The loosely dashed black line is the distribution of new arrivals $F=U n i f[0,1]$, the dotted blue line is $\boldsymbol{G}(\cdot ; \hat{\theta})$, and the solid red line is $\boldsymbol{G}(\cdot ; \hat{\hat{\theta}})$ for $\hat{\hat{\theta}}>\hat{\theta}$.

$\Theta$ but determined by the queuing $\operatorname{CDF} Q_{d}^{*}$ and the rationing probability $\alpha\left(\cdot ; Q_{d}^{*}, \Psi_{d}^{*}\right)$. Formally,

$$
\begin{aligned}
\boldsymbol{U}_{\gamma}^{d} & =\int_{\theta_{d}^{*}(\gamma)}^{1}\left[\omega \alpha\left(\omega ; Q_{d}^{*}, \Psi_{d}^{*}\right)+(1-\gamma) \boldsymbol{U}_{\gamma}^{d}\left(1-\alpha\left(\omega ; Q_{d}^{*}, \Psi_{d}^{*}\right)\right)\right] d Q_{d}^{*}(\omega) \\
& =\int_{\hat{\theta}}^{1} \omega \boldsymbol{G}(\omega ; \hat{\theta})+(1-\gamma) \boldsymbol{U}_{\gamma}^{d} \boldsymbol{G}(\hat{\theta} ; \hat{\theta}),
\end{aligned}
$$

evaluated at $\hat{\theta}=\theta_{d}^{*}(\gamma)$. Thus, the expected payoff in a directed search SME is equivalent to the expected payoff in the hypothetical search market with $\hat{\theta}=\theta_{d}^{*}(\gamma)$, i.e., $\boldsymbol{U}_{\gamma}^{d}=\mathbb{U}\left(\theta_{d}^{*}(\gamma)\right)$.

We can thus compare directed search and random search protocols by focusing on the hypothetical market. Since $\mathbb{U}(\hat{\theta})$ is a decreasing function, $\boldsymbol{U}_{\gamma}^{r}>\boldsymbol{U}_{\gamma}^{d}$ if, and only if, $\theta_{d}^{*}(\gamma)>\theta_{r}^{*}(\gamma)$. In other words, random search yields a higher equilibrium payoff and social welfare than directed search because the agents are less picky when search is random. Consider the simplest case with $\gamma=1$. When search is random, each agent is willing to match to any object because rejecting would yield a payoff of 0 . Hence, when $\gamma=1$, random search achieves the efficient outcome. In contrast, when search is directed, each agent is willing to forsake a guaranteed match with a low quality object for the chance to match with a high quality object with probability less than one. Hence, $0=\theta_{r}^{*}(1)<\theta_{d}^{*}(1)$ and directed search yields a less efficient outcome than random search.

However, as $\gamma$ decreases, $\theta_{r}^{*}(\gamma)$ increases (Corollary 1) while $\theta_{d}^{*}(\gamma)$ remains constant (Corollary 2). Yet, Theorem 1 implies that the agents are always less picky when search is random. Why? 
When search is random, an agent's pickiness is characterized by the fixed point of $(1-\gamma) \mathbb{U}$, the inter-temporal trade-off between her current (and therefore guaranteed) match and her value from searching for potentially better matches in the future. In contrast, when search is directed, an agent's pickiness is characterized by the fixed point of $\mathbb{U}$, the contemporaneous trade-off between a guaranteed match today and her value from searching for potentially better matches today. As an agent facing the former trade-off has to discount her value of search by $1-\gamma$, she is always less picky when search is random. Consequently, random search yields a higher equilibrium payoff than directed search.

\section{Search with Ex-post Transfers}

This section extends the basic search model with non-transferable utility in two dimensions: first, I consider a model where both agents and objects are strategic. Second, I allow for expost transfers. However, the terminology of agents and objects is cumbersome when both are strategic. Henceforth, I consider a labor market framework in which firms (she) play the role of agents, and workers (he) play the role of objects. The exposition stays as close as possible to the previous section.

\subsection{Setup}

In each period, the firms on one side of the search market are paired to the workers on the other side. The workers differ in the level of output they produce. In particular, a worker of productivity type $\theta \in \Theta$ can produce $\theta$ units of output.

Once a firm pairs to a worker, the firm observes the worker's type $\theta$. If both agree to match at a wage of $w \in \mathbb{R}$, then both exit the market and the firm gets a payoff of $\theta-w$ while the worker gets $w$. If at least one of them declines to match, they both return to the search market. The inflow and outflow of firms and workers follows the same dynamics as the respective inflow and outflow of agents and objects in the basic model with non-transferable utility.

\subsection{Random Search Protocol with Ex-post Transfers}

Under a random search protocol, a firm observes a worker's productivity only after being paired. Prior to a pairing, a firm only knows the prevailing endogenously determined market composition $\Psi \triangleq\left\langle G, M^{a}, M^{o}\right\rangle$ where $G$ is the productivity distribution of the workers in the market, $M^{a}$ is the total mass of firms in the market, and $M^{o}$ is the total mass of workers in the 
market. In each period, firms and workers are randomly paired so that the maximal number of pairs is achieved. As there are (weakly) more firms than workers, each worker gets paired to a single firm with probability 1 whereas each firm gets paired to a single worker with probability $M^{o} / M^{a}$.

Once a firm and a worker are paired, the firm observes the worker's productivity $\theta$. If the pair agree to match, they bargain over wages and exit the market. If either the firm or the worker decline to match, both return to the market and search again next period with probability $1-\gamma$.

For a given market composition $\Psi$, let $\mathcal{U}^{r}(\Psi)$ be a firm's value from searching randomly. Similarly, let $\mathcal{V}^{r}(\theta ; \Psi)$ be the value of random search for a worker with productivity $\theta \in \Theta$. I assume that transfers are determined ex-post using a Nash bargaining solution. Hence, the bargained wage between a firm and a type $\theta$ worker is

$$
w^{r}(\theta ; \Psi)=\underset{w \in \mathbb{R}}{\arg \max }\left(\theta-w-(1-\gamma) \mathcal{U}^{r}(\Psi)\right)^{\rho}\left(w-(1-\gamma) \mathcal{V}^{r}(\theta ; \Psi)\right)^{1-\rho}
$$

where $\rho \in(0,1)$ is the bargaining power of firms. In particular,

$$
w^{r}(\theta ; \Psi)=(1-\rho)\left(\theta-(1-\gamma) \mathcal{U}^{r}(\Psi)\right)+\rho(1-\gamma) \mathcal{V}^{r}(\theta ; \Psi)
$$

Suppose a worker of type $\theta \in \Theta$ leaves the market only though a match with a firm. Then,

$$
\mathcal{V}^{r}(\theta ; \Psi)=w^{r}(\theta ; \Psi)=\frac{(1-\rho)\left(\theta-(1-\gamma) \mathcal{U}^{r}(\Psi)\right)}{1-\rho(1-\gamma)}
$$

where the second equality follows from (11). It must be sequentially rational for a worker of type $\theta$ to match with a firm, i.e., $w^{r}(\theta ; \Psi) \geq(1-\gamma) \mathcal{V}^{r}(\theta ; \Psi)$. It must also be sequentially rational for a firm to match with a worker of type $\theta$, i.e., $\theta-w^{r}(\theta ; \Psi) \geq(1-\gamma) \mathcal{U}^{r}(\Psi)$. Using the expression in (12) and the sequential rationality inequalities, a worker of type $\theta$ exists the market only through a match if a non-negative surplus condition $\theta \geq(1-\gamma) \mathcal{U}^{r}(\Psi)$ holds. Furthermore, it is straightforward to check that if $\theta>(1-\gamma) \mathcal{U}^{r}(\Psi)$, the worker leaves only through a match in equilibrium.

Similarly, suppose a worker of type $\theta \in \Theta$ leaves the market only though death. Then, $\mathcal{V}^{r}(\theta ; \Psi)=0$. It must be sequentially rational for either firms or workers to decline to match. A worker of type $\theta$ declines to match with a firm if $w^{r}(\theta ; \Psi) \leq 0$. A firm declines to match with a worker of type $\theta$ if $\theta-w^{r}(\theta ; \Psi) \leq(1-\gamma) \mathcal{U}^{r}(\Psi)$. Using the expression in (11) and the 
sequential rationality inequalities, we get a non-positive surplus condition $\theta \leq(1-\gamma) \mathcal{U}^{r}(\Psi)$. Furthermore, it is straightforward to check that if $\theta<(1-\gamma) \mathcal{U}^{r}(\Psi)$, the worker leaves only through death in equilibrium.

Therefore, there exists a cutoff $\overline{\bar{\theta}}(\Psi)=(1-\gamma) \mathcal{U}^{r}(\Psi)$ such that all workers of type $\theta>\overline{\bar{\theta}}(\Psi)$ leave the market through a match with a firm while all workers of type $\theta<\overline{\bar{\theta}}(\Psi)$ leave the market through death.

Definition 3 A random search SME with transfers is given by a cutoff $\theta_{r} \in \Theta$ and a market composition $\Psi_{r}$ such that

(i) $\theta_{r}=\overline{\bar{\theta}}\left(\Psi_{r}\right)$, and

(ii) $\Psi_{r}=\left\langle\boldsymbol{G}\left(\cdot ; \theta_{r}\right), \boldsymbol{M}^{a}\left(\theta_{r}\right), \boldsymbol{M}^{o}\left(\theta_{r}\right)\right\rangle$ as defined by $(6)-(8)$.

In other words, a stationary equilibrium for random search with transfers is characterized by a pair $\left(\theta_{r}, \Psi_{r}\right)$ such that $(i)$ given the market composition $\Psi_{r}$, it is sequentially rational for

each firm to match with a worker of type $\theta>\theta_{r}$ at wage $\frac{(1-\rho)\left(\theta-\theta_{r}\right)}{1-\rho(1-\gamma)}$ (from (12)) and to decline to match with a worker of type $\theta<\theta_{r}$, and $(i i)$ the market composition $\Psi_{r}$ is consistent with a steady state outcome when all firms use the cutoff $\theta_{r}$.

\section{Proposition 3}

There is a unique random search $S M E$ with transfers given by $\left(\theta_{r}^{* *}, \Psi_{r}^{* *}\right)$. The cutoff $\theta_{r}^{* *}$ satisfies the indifference condition

$$
\theta_{r}^{* *}=\frac{k_{o} \rho \gamma(1-\gamma)}{k_{a}(1-\rho(1-\gamma))-k_{o}(1-\gamma)(1-\rho)\left(1-F\left(\theta_{r}^{* *}\right)\right)} \int_{\theta_{r}^{* *}}^{1} \omega d F(\omega)
$$

Proof. Fix some market composition $\Psi \triangleq\left\langle G, M^{a}, M^{o}\right\rangle$. Given the firm's sequential rational cutoff is $\overline{\bar{\theta}}(\Psi)$, her value from random search is given by

$$
\begin{aligned}
\mathcal{U}^{r}(\Psi) & =\frac{M^{o}}{M^{a}} \int_{\overline{\bar{\theta}}(\Psi)}^{1} \omega-w^{r}(\omega ; \Psi) d G(\omega)+\left(1-\frac{M^{o}}{M^{a}}+\frac{M^{o}}{M^{a}} G(\overline{\bar{\theta}}(\Psi))\right)(1-\gamma) \mathcal{U}^{r}(\Psi) \\
& =\frac{M^{o}}{M^{a}} \int_{\overline{\bar{\theta}}(\Psi)}^{1} \frac{\omega \rho \gamma+(1-\rho)(1-\gamma) \mathcal{U}^{r}(\Psi)}{1-\rho(1-\gamma)} d G(\omega)+\left(1-\frac{M^{o}}{M^{a}}+\frac{M^{o}}{M^{a}} G(\overline{\bar{\theta}}(\Psi))\right)(1-\gamma) \mathcal{U}^{r}(\Psi) \\
& =\frac{\xi \int_{\overline{\bar{\theta}}(\Psi)}^{1} \omega d G(\omega)}{\gamma+(1-\gamma) \xi(1-G(\overline{\bar{\theta}}(\Psi)))},
\end{aligned}
$$


where $\xi=\frac{M^{o}}{M^{a}}\left(\frac{\rho \gamma}{1-\rho(1-\gamma)}\right)$. The first equality follows from substituting $w^{r}$ by the expression in $(12)$.

The pair $\left(\theta_{r}, \Psi_{r}\right)$ constitutes an SME if $\theta_{r}=\overline{\bar{\theta}}\left(\Psi_{r}\right)$ and $\Psi_{r}=\left\langle\boldsymbol{G}\left(\cdot ; \theta_{r}\right), \boldsymbol{M}^{a}\left(\theta_{r}\right), \boldsymbol{M}^{o}\left(\theta_{r}\right)\right\rangle$. Substituting in the market composition expressions in (6)-(8) simplifies the value function to

$$
\mathcal{U}^{r}\left(\Psi_{r}\right)=\frac{k_{o} \rho \gamma}{k_{a}(1-\rho(1-\gamma))-k_{o}(1-\gamma)(1-\rho)\left(1-F\left(\theta_{r}\right)\right)} \int_{\theta_{r}}^{1} \omega d F(\omega)
$$

Since the firm's cutoff strategy for a given market composition $\Psi$ is characterized by the indifference condition $\overline{\bar{\theta}}(\Psi)=(1-\gamma) \mathcal{U}^{r}(\Psi)$,

$$
\theta_{r}=\frac{k_{o} \rho \gamma(1-\gamma)}{k_{a}(1-\rho(1-\gamma))-k_{o}(1-\gamma)(1-\rho)\left(1-F\left(\theta_{r}\right)\right)} \int_{\theta_{r}}^{1} \omega d F(\omega)
$$

Recall the function $\mathbb{U}(\theta)=\int_{\theta}^{1} \omega d F(\omega)$ which is continuous and strictly decreasing in $\theta$. Let $\mathbb{H}: \Theta \rightarrow(0,1]$ be given by

$$
\mathbb{H}(\theta)=\frac{k_{o} \gamma}{k_{a}(1-\rho(1-\gamma))-k_{o}(1-\gamma)(1-\rho)(1-F(\theta))}
$$

which is also a continuous and strictly decreasing function of $\theta$. Hence, there is a unique fixed point to the function $(1-\gamma) \rho \mathbb{H} \cdot \mathbb{U}$ given by $\theta_{r}^{* *}$. The market composition $\Psi_{r}^{* *}$ is then derived from the cutoff $\theta_{r}^{* *}$ using (6)-(8)

Similar to the case of random search with non-transferable utility, a firm who searches randomly faces an inter-temporal trade off; she can either settle for a worker she is currently paired to or she can continue randomly searching for a worker with a higher productivity. However, a firm who declines to match today can continue to search only if she survives into the next period. Thus, her continuation value of search must be discounted by $1-\gamma$. The indifference condition (13) captures the resolution of this inter-temporal trade off. The cutoff $\theta_{r}^{* *}$ is the lowest productivity worker that firms are willing to hire.

For a given $(\rho, \gamma) \in(0,1)^{2}$, let $\theta_{r}^{* *}(\rho, \gamma)$ be the unique equilibrium cutoff satisfying the indifference condition (13). Let $\mathcal{U}_{\rho, \gamma}^{r}=\frac{\theta_{r}^{* *}(\rho, \gamma)}{1-\gamma}$ be the firm's unique equilibrium value of random search.

As a firm's bargaining power increases, she extracts more of the surplus generated by a match. In particular, her ex-post payoff becomes more and more sensitive to the worker's 
productivity. Thus, as $\rho$ increases, the firms become more picky. In the limit when the firms have all the bargaining power, the workers are effectively reduced into the non-strategic objects from the basic model. The firms extract all the surplus and the equilibrium outcomes under transferable and non-transferable utility coincide.

Corollary 3 The cutoff $\theta_{r}^{* *}(\rho, \gamma)$ and the firms' value of random search $\mathcal{U}_{\rho, \gamma}^{r}$ are increasing in $\rho$. Furthermore, $\lim _{\rho \rightarrow 1} \theta_{r}^{* *}(\rho, \gamma)=\theta_{r}^{*}(\gamma)$ and $\lim _{\rho \rightarrow 1} \mathcal{U}_{\rho, \gamma}^{r}=\boldsymbol{U}_{\gamma}^{r}$.

Unlike the case of non-transferable utility, the comparative statics with respect to $\gamma$ is ambiguous and non-monotone. Under Nash bargaining, the discounted continuation values of the players are the disagreement points. As $\gamma$ decreases, the firms disagreement point improves but the same is true for the workers. Which party comes out on top depends on the relative bargaining power of the players.

Nonetheless, if the firms don't have full bargaining power, the random search equilibrium outcome converges to a competitive market outcome as the cost of searching vanishes; in the limit, firms hire all workers at wages $w^{r}(\theta)=\theta$ and the outcome is efficient. This observation is consistent with Lauermann (2013) who shows more generally that outcomes in random search and bargaining games converge to competitive market outcomes if, and only if, there is competitive pressure in the search market. One such competitive pressure is that both sides of the market have bargaining power. ${ }^{15}$ In contrast, if the firms have full bargaining power, the outcome of the random search market with transfers converges to the inefficient directed search outcome with non-transferable utility as the search cost vanishes.

Corollary 4 As $\gamma \rightarrow 0$, the random search SME with transfers converges to a competitive market outcome if, and only if, $\rho<1$.

Similar to a random search market with non-transferable utility, the unique equilibrium outcome in a random search market with transferable utility is inefficient. The efficient outcome would be for all firms to match with any type of worker. However, matching with all worker types is not sequentially rational for any single firm. In equilibrium, a firm instead chooses to match to all types above $\theta_{r}^{* *}(\rho, \gamma)$ after taking into account the negative externalities that other firms impose on the market composition. However, firms do not fully internalize the negative externalities of their own strategic choices. Furthermore, since bargaining is over the ex-post surplus, wages also do not fully internalize the negative externalities. Hence, the random search SME with transfers is also inefficient.

\footnotetext{
${ }^{15}$ Other forms of competitive pressure are asymmetric information with noisy signals, and one firm bargaining with multiple workers simultaneously.
} 


\subsection{Directed Search Protocol with Ex-post Transfers}

Similar to the basic model, the search market is further subdivided into a continuum of submarkets. A sub-market $S(\theta)$ exclusively contains all workers in the market with productivity $\theta$. Firms can distinguish between all the sub-markets and can choose which sub-market to search in for a given period. For instance, we can think of $S(\theta)$ as a university that has a reputation of producing workers of type $\theta$. Firms can then choose in which universities they recruit for employees.

Let $Q$ be the absolutely continuous queuing CDF with density function $q$. Given a market composition $\Psi=\left\langle G, M^{a}, M^{o}\right\rangle$, sub-market $S(\theta)$ has a "mass" of $M^{o} g(\theta)$ workers and a queue length of $M^{a} q(\theta)$ firms.

In each period, firms and workers within a sub-market are paired randomly so that the maximal number of pairs is achieved. Let $\alpha(\theta ; Q, \Psi)=\min \left\{\frac{M^{o} g(\theta)}{M^{a} q(\theta)}, 1\right\}$ be the firm match probability, the probability that a firm in sub-market $S(\theta)$ gets a match in a given period. Similarly, let $\beta(\theta ; Q, \Psi)=\min \left\{\frac{M^{a} q(\theta)}{M^{o} g(\theta)}, 1\right\}$ be the worker match probability, the probability that a worker in sub-market $S(\theta)$ gets matched in a given period.

For a given market composition $\Psi$ and queuing $\operatorname{CDF} Q$, let $\mathcal{U}^{d}(\theta ; Q, \Psi)$ be a firm's value from directing her search to sub-market $S(\theta)$. Similarly, let $\mathcal{V}^{d}(\theta ; Q, \Psi)$ be the value function for a worker in sub-market $S(\theta)$.

On the equilibrium path where $\theta \in S u p p(q),{ }^{16}$ a firm and worker determine wages using a Nash bargaining solution concept. Let

$$
w^{d}(\theta ; Q, \Psi)=(1-\rho)\left(\theta-(1-\gamma) \mathcal{U}^{d}(\theta ; Q, \Psi)\right)+\rho(1-\gamma) \mathcal{V}^{d}(\theta ; Q, \Psi)
$$

be the on-path Nash bargaining solution between a firm and a type $\theta$ worker. The value of search for a worker in an on-path sub-market $S(\theta)$ can then be written as

$$
\begin{aligned}
\mathcal{V}^{d}(\theta ; Q, \Psi)= & \beta(\theta ; Q, \Psi) w^{d}(\theta ; Q, \Psi)+(1-\beta(\theta ; Q, \Psi))(1-\gamma) \mathcal{V}^{d}(\theta ; Q, \Psi) \\
= & \frac{\beta(\theta ; Q, \Psi)(1-\rho)\left(\theta-(1-\gamma) \mathcal{U}^{d}(\theta ; Q, \Psi)\right)}{\gamma+\beta(\theta ; Q, \Psi)(1-\gamma)(1-\rho)}
\end{aligned}
$$

\footnotetext{
${ }^{16} \operatorname{Supp}(q)$ is the support of the density $q$, i.e., $\{\theta: q(\theta)>0\}$. Hence, $\operatorname{Supp}(q)$ are the sub-markets firms enter.
} 
Similarly, the value of search for a firm in an on-path sub-market $S(\theta)$ can be written as

$$
\begin{aligned}
\mathcal{U}^{d}(\theta ; Q, \Psi) & =\alpha(\theta ; Q, \Psi)\left(\theta-w^{d}(\theta ; Q, \Psi)\right)+(1-\alpha(\theta ; Q, \Psi))(1-\gamma) \mathcal{U}^{d}(\theta ; Q, \Psi) \\
& =\frac{\rho \alpha(\theta ; Q, \Psi) \theta}{\gamma+(1-\gamma)(\rho \alpha(\theta ; Q, \Psi)+(1-\rho) \beta(\theta ; Q, \Psi))} .
\end{aligned}
$$

Off the equilibrium path where $\theta \notin \operatorname{Supp}(q)$, a worker's payoff is $\mathcal{V}(\theta ; Q, \Psi)=0$. If a firm deviates to an off-path sub-market, then she is guaranteed an immediate match and gets a deviation payoff of $\mathcal{U}^{d}(\theta ; Q, \Psi)=\theta-w^{d}(\theta ; Q, \Psi)$. However, I do not require off-path wages to also be solutions to a Nash bargaining problem. Instead, I assume that wages are continuous (both on and off-path), i.e., I focus on continuous equilibria in which off-path wages are limit points of on-path wages. Furthermore, I assume that off-path wages do not change too fast.

\section{Assumption 1}

(i) $w^{d}(\theta ; Q, \Psi)$ is continuous in $\theta$.

(ii) For all $\theta \in \operatorname{Supp}(q), w^{d}, \mathcal{V}^{d}$, and $\mathcal{U}^{d}$ are given by (14), (15), and (16) respectively.

(iii) For all $\theta^{\prime}, \theta^{\prime \prime} \notin \operatorname{Supp}(q),\left|w^{d}\left(\theta^{\prime} ; Q, \Psi\right)-w^{d}\left(\theta^{\prime \prime} ; Q, \Psi\right)\right|<\left|\theta^{\prime}-\theta^{\prime \prime}\right|$.

In Online Appendix B, I relax this assumption and characterize all equilibrium outcomes when both on and off-path wages are determined by Nash bargaining. However, the latter approach makes the problem less tractable. ${ }^{17}$

Definition 4 A directed search $S M E$ with transfers is given by a pair $\left(Q_{d}, \Psi_{d}\right)$ such that there exists a wage function $w^{d}\left(\cdot ; Q_{d}, \Psi_{d}\right): \Theta \rightarrow \mathbb{R}$ that satisfies Assumption 1 and

(i) $Q_{d}(\theta)=\int_{0}^{\theta} q_{d}(\omega) d \omega$ for all $\theta \in \Theta$ with $Q_{d}(1)=1$,

\footnotetext{
${ }^{17}$ When wages are determined by Nash bargaining everywhere, the wages indirectly depend on the worker match probability $\beta(\cdot ; Q, \Psi)$. Suppose firms only enter sub-markets above a cutoff $\theta^{\prime}$ in equilibrium. Then for all $\theta$ in a left neighborhood of $\theta^{\prime}, S(\theta)$ is off-path and thus $\beta(\theta ; Q, \Psi)=0$. If $\beta(\cdot ; Q, \Psi)$ discretely jumps up in a right neighborhood of $\theta^{\prime}$, then so do the Nash bargaining wages, i.e., $w^{d}\left(\theta^{\prime}-\epsilon ; Q, \Psi\right) \ll w^{d}\left(\theta^{\prime}+\epsilon ; Q, \Psi\right)$ for some $\epsilon>0$ small enough. However, by entering $S\left(\theta^{\prime}+\epsilon\right)$ instead of $S\left(\theta^{\prime}-\epsilon\right)$, firms get a marginal gain in productivity but a discrete loss in wages which makes deviating to $S\left(\theta^{\prime}-\epsilon\right)$ profitable. To prevent such downward deviations, the worker match probability cannot jump at the cutoff. Hence, there is some interval $\left(\theta^{\prime}, \theta^{\prime \prime}\right)$ over which firms are rationed and workers are on the long side. This however complicates the market composition which would no longer be piecewise linear. Nonetheless, I show that an equilibrium exists and derive a general characterization for it. Furthermore, I show that if firm bargaining power is high enough, or if search is costly enough, the main result of this section continues to hold.
} 
(ii) $q_{d}(\theta)>0$ if and only if $\theta \in \arg \max _{\theta^{\prime} \in \Theta} \mathcal{U}^{d}\left(\theta^{\prime} ; Q_{d}, \Psi_{d}\right)$, and

(iii) $\Psi_{d}=\left\langle\boldsymbol{G}\left(\cdot ; \beta\left(\cdot ; Q_{d}, \Psi_{d}\right)\right), \boldsymbol{M}^{a}\left(\beta\left(\cdot ; Q_{d}, \Psi_{d}\right)\right), \boldsymbol{M}^{o}\left(\beta\left(\cdot ; Q_{d}, \Psi_{d}\right)\right)\right\rangle$ as given by $(3)-(5)$.

In other words, a stationary equilibrium for directed search with transfers is characterized by a pair $\left(Q_{d}, \Psi_{d}\right)$ such that there exists a wage function $w^{d}$ which $(i)$ given $\left(Q_{d}, \Psi_{d}\right), w^{d}$ is derived by Nash bargaining on-path and has nice smoothness properties off-path, $(i i)$ given $w^{d}$, the queueing $\operatorname{CDF} Q_{d}$ is consistent with the firms entering only the sub-markets that maximize their profits, and (iii) the market composition $\Psi_{d}$ is consistent with a steady state outcome given by (3)-(5).

\section{Proposition 4}

Under Assumption 1, there exists a directed search SME given by $\left(Q_{d}^{* *}, \Psi_{d}^{* *}\right)$ where

(i) $\Psi_{d}^{* *}=\left\langle\boldsymbol{G}\left(\cdot ; \theta_{d}^{* *}\right), \boldsymbol{M}^{a}\left(\theta_{d}^{* *}\right), \boldsymbol{M}^{o}\left(\theta_{d}^{* *}\right)\right\rangle$,

(ii) $Q_{d}^{* *}(\theta)=\left\{\begin{array}{cc}0 & \text { if } \quad \theta \leq \theta_{d}^{* *} \\ \int_{\theta_{d}^{* *}}^{\theta} \frac{k_{o} \gamma\left(\omega-\rho(1-\gamma) \theta_{d}^{* *}\right)}{\left(k_{a}-k_{o}(1-\gamma)\left(1-F\left(\theta_{d}^{* *}\right)\right) \theta_{d}^{* *}(1-\rho(1-\gamma))\right.} d F(\omega) & \text { for } \quad \theta \geq \theta_{d}^{* *}\end{array}\right.$

(iii) $\mathcal{U}^{d}\left(\theta ; Q_{d}^{* *}, \Psi_{d}^{* *}\right)=\rho \theta_{d}^{* *}, \forall \theta \geq \theta_{d}^{* *}$

The cutoff $\theta_{d}^{* *} \in \Theta$ is characterized by

$$
\theta_{d}^{* *}=\frac{k_{o} \gamma}{k_{a}(1-\rho(1-\gamma))-k_{o}(1-\gamma)(1-\rho)\left(1-F\left(\theta_{d}^{* *}\right)\right)} \int_{\theta_{d}^{* *}}^{1} \omega d F(\omega)
$$

Proof. If the pair $\left(Q_{d}, \Psi_{d}\right)$ constitute a directed search SME, there exists a cutoff $\theta_{d} \in \Theta$ such that firms only enter sub-markets $S(\theta)$ with $\theta>\theta_{d}$. I will look for equilibrium where $\beta\left(\theta ; Q_{d}, \Psi_{d}\right)=1$ for all $\theta>\theta_{d}$. Hence, in an on-path sub-market $S(\theta)$, the firm's payoff is given by

$$
\mathcal{U}^{d}(\theta ; Q, \Psi)=\frac{\rho \alpha(\theta ; Q, \Psi) \theta}{\gamma+(1-\gamma)(\rho \alpha(\theta ; Q, \Psi)+1-\rho)} \leq \rho \theta
$$


and, the wage is given by

$$
w^{d}(\theta ; Q, \Psi)=\frac{\theta(1-\rho)}{\gamma+(1-\gamma)\left(\rho \alpha\left(\theta ; Q_{d}, \Psi_{d}\right)+1-\rho\right)}
$$

Off-path, a firm's payoff by entering a sub-market $S(\theta)$ with $\theta<\theta_{d}$ is given by $\mathcal{U}^{d}\left(\theta ; Q_{d}, \Psi_{d}\right)=$ $\theta-w^{d}\left(\theta ; Q_{d}, \Psi_{d}\right)$ which is increasing in $\theta$ given Assumption 1. Furthermore, by the continuity of wages, $\lim _{\theta \uparrow \theta_{d}} \mathcal{U}^{d}\left(\theta ; Q_{d}, \Psi_{d}\right)=\theta_{d}-w^{d}\left(\theta_{d} ; Q_{d}, \Psi_{d}\right)$.

Suppose $\lim _{\theta \downarrow \theta_{d}} \alpha\left(\theta ; Q_{d}, \Psi_{d}\right)=\bar{\alpha}<1$. Then the limit point of a firm's on-path payoff is

$$
\lim _{\theta \downarrow \theta_{d}} \mathcal{U}^{d}(\theta ; Q, \Psi)=\frac{\rho \bar{\alpha} \theta_{d}}{\gamma+(1-\gamma)(\rho \bar{\alpha}+1-\rho)}
$$

In contrast, the limit point of the firm's off-path payoff is given by

$\lim _{\theta \uparrow \theta_{d}} \mathcal{U}^{d}(\theta ; Q, \Psi)=\theta_{d}-w^{d}\left(\theta_{d} ; Q_{d}, \Psi_{d}\right)=\theta_{d}-\frac{\theta_{d}(1-\rho)}{\gamma+(1-\gamma)(\rho \bar{\alpha}+1-\rho)}>\frac{\rho \bar{\alpha} \theta_{d}}{\gamma+(1-\gamma)(\rho \bar{\alpha}+1-\rho)}$

which implies that for $\epsilon>0$ small enough, the firms would strictly prefer to deviate to an off-path sub-market $S(\theta)$ with $\theta \in\left(\theta_{d}-\epsilon, \theta_{d}\right)$. Hence, $\bar{\alpha}=1$ and

$$
\lim _{\theta \uparrow \theta_{d}} \mathcal{U}^{d}\left(\theta ; Q_{d}, \Psi_{d}\right)=\rho \theta_{d}=\lim _{\theta \downarrow \theta_{d}} \mathcal{U}^{d}\left(\theta ; Q_{d}, \Psi_{d}\right)
$$

As the firms must be indifferent among all the sub-markets they enter, $\mathcal{U}^{d}\left(\theta^{\prime \prime} ; Q_{d}, \Psi_{d}\right)=$ $\mathcal{U}^{d}\left(\theta^{\prime} ; Q_{d}, \Psi_{d}\right)$ for all $\theta^{\prime \prime}>\theta^{\prime}>\theta_{d}$. Thus, $\mathcal{U}^{d}\left(\theta ; Q_{d}, \Psi_{d}\right)=\rho \theta_{d}$ for all $\theta \geq \theta_{d}$.

The indifference condition $\mathcal{U}^{d}\left(\theta ; Q_{d}, \Psi_{d}\right)=\rho \theta_{d}$ for all $\theta>\theta_{d}$ necessitates that $\alpha\left(\theta ; Q_{d}, \Psi_{d}\right)=$ $\frac{\theta_{d}(1-\rho(1-\gamma))}{\theta-\rho(1-\gamma) \theta_{d}}$ for all $\theta>\theta_{d}$. Because $\alpha\left(\theta ; Q_{d}, \Psi_{d}\right)<1$ for all $\theta>\theta_{d}$, firms must be on the long-side of each sub-market above the cutoff. By definition, we also have that $\alpha\left(\theta ; Q_{d}, \Psi_{d}\right)=\frac{g_{d}(\theta)}{q_{d}(\theta)} \zeta_{d}$ for all $\theta>\theta_{d}$, where $\zeta_{d}=\frac{M_{d}^{o}}{M_{d}^{a}}$. Equating these two expressions for the firm match probability and 
integrating over the interval $\left[\theta_{d}, 1\right]$ gives

$$
\begin{gathered}
\theta_{d}(1-\rho(1-\gamma)) \underbrace{\int_{\theta_{d}}^{1} q_{d}(\theta) d \theta}_{=1}=\zeta_{d} \int_{\theta_{d}}^{1} \theta d G_{d}(\theta)-\zeta_{d} \rho(1-\gamma) \theta_{d}\left(1-G_{d}\left(\theta_{d}\right)\right) \\
\Leftrightarrow \theta_{d}=\frac{\zeta_{d} \int_{\theta_{d}}^{1} \theta d G_{d}(\theta)}{1-\rho(1-\gamma)+\zeta_{d} \rho(1-\gamma)\left(1-G_{d}\left(\theta_{d}\right)\right)}
\end{gathered}
$$

The market composition $\Psi_{d}$ must be consistent with $\left\langle\boldsymbol{G}\left(\cdot ; \theta_{d}\right), \boldsymbol{M}^{a}\left(\theta_{d}\right), \boldsymbol{M}^{o}\left(\theta_{d}\right)\right\rangle$ as given by (6)-(8). Substituting in these expressions for $G_{d}, M_{d}^{a}$, and $M_{d}^{o}$ gives

$$
\theta_{d}=\frac{k_{o} \gamma}{k_{a}(1-\rho(1-\gamma))-k_{o}(1-\gamma)(1-\rho)\left(1-F\left(\theta_{d}\right)\right)} \int_{\theta_{d}}^{1} \omega d F(\omega)
$$

Recall the functions $\mathbb{U}: \Theta \rightarrow \Theta$ and $\mathbb{H}: \Theta \rightarrow(0,1]$ which are both continuous and strictly decreasing in $\theta$. Hence, there is a unique fixed point to the function $\mathbb{H} \cdot \mathbb{U}$ given by $\theta_{d}^{* *}$.

The market composition $\Psi_{d}^{* *}$ is then derived from the cutoff $\theta_{d}^{* *}$ using (6)-(8). Similarly, the queueing $\mathrm{CDF}$ is pinned down from the agent match probability with

$$
\alpha\left(\theta ; Q_{d}^{* *}, \Psi_{d}^{* *}\right)=\frac{\boldsymbol{M}^{o}\left(\theta_{d}^{* *}\right) \boldsymbol{g}\left(\theta ; \theta_{d}^{* *}\right)}{\boldsymbol{M}^{a}\left(\theta_{d}^{* *}\right) q_{d}^{* *}(\theta)}=\frac{\theta_{d}^{* *}(1-\rho(1-\gamma))}{\theta-\rho(1-\gamma) \theta_{d}^{* *}}
$$

for all $\theta>\theta_{d}^{* *}$.

Remark 1 Proposition 4 characterizes an equilibrium outcome when all on-path sub-markets are congested (more firms than workers). It may be possible that there is another equilibrium in which some of the intermediate productivity sub-markets are in fact thin (more workers than firms). I explore such an equilibrium outcome when Assumption 1 is relaxed (in the online appendix Mekonnen (2018)).

A firm faces a contemporaneous trade off; she can either settle for a worker she can match to without waiting or she can queue in a congested sub-market for the possibility of matching with a more productive worker. The indifference condition (17) captures the resolution of this contemporaneous trade off. The cutoff $\theta_{d}^{* *}$ is the highest productivity worker that firms can get without waiting in a congested sub-market.

For a given $(\rho, \gamma) \in(0,1)^{2}$, let $\theta_{d}^{* *}(\rho, \gamma)$ be the equilibrium cutoff satisfying the indifference condition (17). Let $\mathcal{U}_{\rho, \gamma}^{d}=\rho \theta_{d}^{* *}(\rho, \gamma)$ be the firm's equilibrium payoff of directed search. 
As a firm's bargaining power increases, she extracts more of the surplus generated by a successful match. Thus, as $\rho$ increases, the firms become more picky. In the limit when the firms have all the bargaining power, the equilibrium outcomes under transferable and nontransferable utility coincide.

Corollary 5 The cutoff $\theta_{d}^{* *}(\rho, \gamma)$ and the firms' value of directed search $\mathcal{U}_{\rho, \gamma}^{d}$ are increasing in $\rho$. Furthermore, $\lim _{\rho \rightarrow 1} \theta_{d}^{* *}(\rho, \gamma)=\theta_{d}^{*}(\gamma)$ and $\lim _{\rho \rightarrow 1} \mathcal{U}_{\rho, \gamma}^{d}=\boldsymbol{U}_{\gamma}^{d}$.

Unlike the case of non-transferable utility, the comparative statics with respect to $\gamma$ is no longer constant when $\rho<1$. The mass of productive workers with $\theta>\theta_{d}^{* *}$ is unaffected by a decrease in $\gamma$ as all these workers exit the market only through successful matches. However, more of the unmatched firms survive into the subsequent periods, making the congestion worse. Furthermore, a decrease in $\gamma$ improves the discounted continuation value of the workers. Thus, when the productive workers have some bargaining power, they demand higher wages not only because they are relatively rarer but also because they have a better disagreement point.

As a result, if the firms don't have full bargaining power, they become more willing to settle for low productivity workers as the cost of searching decreases. Similar to random search with transfers, the directed search SME with transfers converges to a competitive market outcome when the cost of searching vanishes; in the limit, firms enter all sub-markets and hire all workers at wages $w^{d}(\theta)=\theta$ which is efficient.

Corollary 6 For any $\rho<1$, the cutoff $\theta_{d}^{* *}(\rho, \gamma)$ and the firms'value of directed search $\mathcal{U}_{\rho, \gamma}^{d}$ are increasing in $\gamma$. Furthermore, as $\gamma \rightarrow 0$, the directed search SME with transfers converges to a competitive market outcome if, and only if, $\rho<1$.

Unsurprisingly, the equilibrium outcome in a Proposition 4 is inefficient. The efficient outcome would be for all firms to queue in all sub-markets which is not optimal for any single firm. In equilibrium, a firm instead chooses to queue at any sub-market above $\theta_{d}^{* *}(\rho, \gamma)$ after taking into account the congestion externalities that other firms impose on the market. However, firms do not fully internalize the negative externalities of their own strategic choices. Furthermore, the Nash bargaining protocol only splits the ex-post surplus once a match forms; it does not take into account the search externalities that arise prior to the formation of a match. Hence, the directed search SME with transfers is inefficient.

The result stands in stark contrast to the vast literature on directed search with posted wages. Posted wages provide an ex-ante mechanism for one side of the market to internalize the search frictions. However, the ability to post wages must be complemented with commitment 
power. If the posted wages are non-binding, then one side of the market may find it profitable to renege and propose new wages, undermining the efficiency properties of directed search markets with posted prices.

\subsection{Social Welfare Comparison with Ex-post Transfers}

Similar to the case of non-transferable utility, random and directed search markets with ex-post transfers lead to inefficient equilibrium outcomes. I conclude this section by showing that the inefficiencies created by directed search are worse than the inefficiencies arising from random search. ${ }^{18}$ The intuition is precisely the same as the one for non-transferable utility.

Let $i=r, d$ be an index for random and directed respectively. As in the basic model, welfare is measured by the aggregate value of search for the new entrants. For given parameters $(\rho, \gamma) \in(0,1)^{2}$, let $\mathcal{V}_{\rho, \gamma}^{r}(\theta)$ be the type $\theta$ worker's value of random search derived from $(12)$. Similarly, let $\mathcal{V}_{\rho, \gamma}^{d}(\theta)$ be the type $\theta$ worker's value of directed search derived from (15). The social welfare under protocol $i=r, d$ is then given by

$$
\begin{aligned}
\mathcal{W}_{\rho, \gamma}^{i} & =k_{a} \mathcal{U}_{\rho, \gamma}^{i}+k_{o} \int_{\theta_{i}^{* *}(\rho, \gamma)}^{1} \mathcal{V}_{\rho, \gamma}^{i}(\omega) d F(\omega) \\
& =k_{o} \int_{\theta_{i}^{* *}(\rho, \gamma)}^{1} \omega d F(\omega) .
\end{aligned}
$$

\section{Theorem 2}

For any $(\rho, \gamma) \in(0,1]^{2}, \mathcal{U}_{\rho, \gamma}^{r}>\mathcal{U}_{\rho, \gamma}^{d}$, and $\mathcal{W}_{\rho, \gamma}^{r}>\mathcal{W}_{\rho, \gamma}^{d}$. Furthermore, $\lim _{\gamma \rightarrow 0} \mathcal{U}_{\rho, \gamma}^{r}-\mathcal{U}_{\rho, \gamma}^{d}=0$ and $\lim _{\gamma \rightarrow 0} \mathcal{W}_{\rho, \gamma}^{r}-\mathcal{W}_{\rho, \gamma}^{d}=0$.

Proof. The proof is similar to that of Theorem 1.

\section{Conclusion}

This paper provides a tractable and parsimonious search model to study how changes in the search protocol or the timing of information affect equilibrium outcomes and welfare. The overall theme of the paper is that when agents search for scarce resources of heterogeneous quality, giving agents more flexibility in how they search or more ex-ante information could be detrimental. Specifically, I show that when there are more agents than resources, directed

\footnotetext{
${ }^{18}$ Reader should be aware that the welfare comparisons in this section are not as strong as those of the basic model since the directed search SME with transfers characterized in Proposition 4 is not unique.
} 
search creates worse inefficiencies and leads to lower equilibrium payoffs than random search. While the main result of the paper is framed in a search model with non-transferable utility, I also show that the same conclusions extend into a search model when utility is transferable through Nash bargaining on the equilibrium path.

In the Online Appendix (Mekonnen, 2018), I show that the main insights in this paper are robust to changes in the assumptions made in the model. Specifically, I show that the main result continues to hold for some parameters of the model that violate the scarcity assumption. Additionally, adding a small amount of friction to the matching technology, as is common in the macroeconomics and search literature, does not change the main result.

I also provide a generalization of the model in which objects are both vertically differentiated by quality and horizontally differentiated by attributes, and agents have heterogeneous tastes over the horizontal attributes while also caring about the quality. I show that directed search based only on horizontal attributes is always better than both random search and directed search based on both horizontal attributes and quality. Additionally, I show that if the amount of taste heterogeneity in the market is small, random search is better than directed search based on both horizontal attributes and quality.

Finally, I also consider a partially directed search protocol based on a finite partition of the quality space, and show that welfare is higher under the random search protocol than under a partially directed search protocol for any finite partition.

Of course, there are other search protocols than just random and directed search. For

example, we could allow an agent to direct her search for the first $T$ periods after she enters the market and force her to search randomly thereafter. We could also introduce priority lotteries or formal queues into the market. An interesting avenue for future work would be to adopt a market design approach and characterize the welfare-maximizing search protocol.

\section{Appendix}

\section{Proof of Lemma 1:}

Proof. Suppose there is a solution $\Psi=\left\langle\boldsymbol{G}(\cdot ; \ell), \boldsymbol{M}^{a}(\ell), \boldsymbol{M}^{o}(\ell)\right\rangle$ to the system of functional equations (1)-(2). I will use the necessary conditions associated with such a solution to derive the market composition. 
Fix $\gamma>0$. Since $\ell$ is a probability, for any $\theta^{\prime}<\theta^{\prime \prime}$,

$$
\begin{aligned}
& \boldsymbol{M}^{o}(\ell) \gamma\left(\boldsymbol{G}\left(\theta^{\prime \prime} ; \ell\right)-\boldsymbol{G}\left(\theta^{\prime} ; \ell\right)\right) \\
\leq & \underbrace{\boldsymbol{M}^{o}(\ell) \int_{\theta^{\prime}}^{\theta^{\prime \prime}}((1-\ell(\omega)) \gamma+\ell(\omega)) d \boldsymbol{G}(\omega ; \beta)}_{=k_{o}\left(F\left(\theta^{\prime \prime}\right)-F\left(\theta^{\prime}\right)\right) \text { by }(1)} \\
\leq & \boldsymbol{M}^{o}(\ell)\left(\boldsymbol{G}\left(\theta^{\prime \prime} ; \ell\right)-\boldsymbol{G}\left(\theta^{\prime} ; \ell\right)\right)
\end{aligned}
$$

Evaluating the above inequalities at $\theta^{\prime}=0$ and $\theta^{\prime \prime}=1$ gives $k_{o} \leq \boldsymbol{M}^{o}(\ell) \leq k_{o} \gamma^{-1}$. Additionally, for any $\theta^{\prime}<\theta^{\prime \prime}$,

$$
\gamma\left(F\left(\theta^{\prime \prime}\right)-F\left(\theta^{\prime}\right)\right) \leq \boldsymbol{G}\left(\theta^{\prime \prime} ; \ell\right)-\boldsymbol{G}\left(\theta^{\prime} ; \ell\right) \leq \gamma^{-1}\left(F\left(\theta^{\prime \prime}\right)-F\left(\theta^{\prime}\right)\right) .
$$

Thus, if a steady state market composition exists, $\boldsymbol{G}(\cdot ; \ell)$ is necessarily absolutely continuous with respect to $F$, which is itself absolutely continuous. Hence, $\boldsymbol{G}(\cdot ; \ell)$ must have a density function $\boldsymbol{g}(\cdot ; \ell)$, and $0<\gamma \underline{f} \leq \boldsymbol{g}(\theta ; \ell) \leq \gamma^{-1} \bar{f}<\infty$ for almost all $\theta \in \Theta$.

Furthermore, from (1), we must have $M^{o}(\ell)=k_{o} \mathbb{E}_{G}[\ell+(1-\ell) \gamma]^{-1}$ and

$$
\boldsymbol{g}(\theta ; \ell)=\frac{k_{o}}{M^{o}(\ell)}\left(\frac{f(\theta)}{\ell(\theta)+\gamma(1-\ell(\theta))}\right)=\mathbb{E}_{\boldsymbol{G}}[\ell+(1-\ell) \gamma]\left(\frac{f(\theta)}{\ell(\theta)+\gamma(1-\ell(\theta))}\right)
$$

for almost all $\theta \in \Theta$. Thus,

$$
\boldsymbol{G}(\theta ; \ell)=\mathbb{E}_{\boldsymbol{G}}[\ell+(1-\ell) \gamma] \int_{0}^{\theta} \frac{1}{\ell(\omega)+\gamma(1-\ell(\omega))} d F(\omega) .
$$

However, $\boldsymbol{G}(1 ; \ell)=1$ which is true only if $\mathbb{E}_{\boldsymbol{G}}[\ell+(1-\ell) \gamma]=\mathbb{E}_{F}\left[(\ell+(1-\ell) \gamma)^{-1}\right]^{-1}$. Hence, $\boldsymbol{G}(\cdot ; \ell)$ and $\boldsymbol{M}^{o}(\ell)$ are characterized by (3) and (4). Finally, from (2), we can express $\boldsymbol{M}^{a}(\ell)-\boldsymbol{M}^{o}(\ell)$ as

$$
\gamma^{-1}\left[k_{a}-(1-\gamma) \boldsymbol{M}^{o}(\ell) \int_{\Theta} \ell(\omega) d \boldsymbol{G}(\omega ; \ell)-\boldsymbol{M}^{o}(\ell) \gamma\right]=\gamma^{-1}\left[k_{a}-k_{o}\right]
$$

where the last equality follows from (1). Thus, $\boldsymbol{M}^{a}(\ell)$ is characterized by (5). 


\section{A. Basic Search Model: Extensions}

\section{A.1. Frictional Search}

One of the assumptions in the basic model of Section 1 is that search is frictionless. Specifically, I assumed that if there is a mass $M^{o}$ of objects and a mass $M^{a}$ of agents in a (sub)market, then each object gets paired to an agent with probability $\min \left\{\frac{M^{a}}{M^{o}}, 1\right\}$ while each agent gets paired to an object with probability $\min \left\{\frac{M^{o}}{M^{a}}, 1\right\}$.

In this section of the appendix, I first characterize the unique random and directed search SMEs for a class of frictional pairing technologies. I then show that the main result of Theorem 1 continues to hold when search frictions are small.

Define $t \triangleq \frac{M^{a}}{M^{o}}$ as the "market tightness." Let $x: \mathbb{R}_{+} \rightarrow[0,1]$ be a pairing technology such that given a market tightness $t$, an object gets paired with probability $x(t)$ while an agent gets paired with probability $\frac{x(t)}{t}$. Let $\mathcal{X}$ be the class of pairing technologies that satisfy ${ }^{19}$

1. Regularity: $x(t)$ is continuous, (weakly) increasing, and (weakly) concave in $t$,

2. Feasibility: $x(t) \leq \min \{t, 1\}$, and

3. Limits: $x(0)=0, \lim _{t \rightarrow \infty} x(t)=1$, and $\lim _{t \rightarrow 0} \frac{x(t)}{t}=1$.

This class of matching technologies is common in the labor/macroeconomic search literature. ${ }^{20}$ Here are a few examples of pairing technologies in $\mathcal{X}$.

a. Frictionless pairing technologies: $x(t)=\min \{t, 1\}$,

b. CES pairing technologies: $x(t)=\left(1+t^{\frac{-1}{\phi}}\right)^{-\phi}$ for $\phi>0$, and

c. Urn ball/exponential pairing: $x(t)=t\left(1-\exp \left(\frac{-\phi}{t}\right)\right)$ for $0<\phi \leq 1$.

\section{A.1.1. Frictional Random Search Protocol}

Consider a pairing technology $x \in \mathcal{X}$, a market composition $\Psi=\left\langle G, M^{a}, M^{o}\right\rangle$, and some market tightness $t=M^{a} / M^{o}$. The value of random search is given by

$$
U^{r}(\Psi)=\frac{x(t)}{t} \int_{\Theta} \max \left\{\theta,(1-\gamma) U^{r}(\Psi)\right\} d G(\theta)+\left(1-\frac{x(t)}{t}\right)(1-\gamma) U^{r}(\Psi)
$$

\footnotetext{
${ }^{19}$ The mass of paired objects and paired agents must be the same. If $y(t)$ is the probability that an agent gets paired, $M^{o} x(t)=M^{a} y(t) \Longrightarrow y(t)=\frac{x(t)}{t}$. As $x(t) \leq \min \{t, 1\}, \frac{x(t)}{t}$ is a well-defined probability. Additionally, concavity of $x$ implies $\frac{x(t)}{t}$ is a decreasing function.

${ }^{20}$ See Petrongolo and Pissarides (2001) and Rogerson et al. (2005) for a survey.
} 
The agents' optimal strategy is still given by some cutoff $\bar{\theta}(\Psi)=(1-\gamma) U^{r}(\Psi)$ such that an agent matches to any object of quality $\theta>\bar{\theta}(\Psi)$ and rejects any object of quality $\theta<\bar{\theta}(\Psi)$.

The above expression takes the market composition as exogenous. However, the market composition endogenously depends on the agents' strategy. Given some cutoff strategy $\hat{\theta} \in \Theta$ and market tightness $t$, the market composition $\Psi=\left\langle\boldsymbol{G}(\cdot ; \ell), \boldsymbol{M}^{a}(\ell), \boldsymbol{M}^{o}(\ell)\right\rangle$ can be derived from Lemma 1 with $\ell(\theta)=\mathbb{1}_{[\theta>\hat{\theta}]} x(t) .{ }^{21}$ It is important to note that the market tightness is itself an endogenous variable and must be consistent with the market composition in equilibrium.

Definition A.1 Given a pairing technology $x \in \mathcal{X}$, a random search SME is given by a cutoff $\theta_{r} \in \Theta$, a market composition $\Psi_{r}$, and a market tightness $t_{r}>0$ such that

(i) $\Psi_{r}=\left\langle\boldsymbol{G}(\cdot ; \ell), \boldsymbol{M}^{a}(\ell), \boldsymbol{M}^{o}(\ell)\right\rangle$ is given by $(3)-(5)$ with $\ell(\theta)=\mathbb{1}_{\left[\theta>\theta_{r}\right]} x\left(t_{r}\right)$,

(ii) $\theta_{r}=\bar{\theta}\left(\Psi_{r}\right)$, and

(iii) $t_{r}=\frac{\boldsymbol{M}^{a}(\ell)}{\boldsymbol{M}^{o}(\ell)}$.

To get some intuition for Definition A.1, consider the following iterative process: Take some arbitrary $\Psi_{1}$ and compute $t_{1}$. The agents optimally use the cutoff strategy $\theta_{1}=\bar{\theta}\left(\Psi_{1}\right)$. The cutoff strategy $\theta_{1}$ and the market tightness $t_{1}$ are used to derive a new market composition $\Psi_{2}=\left\langle\boldsymbol{G}(\cdot ; \ell), \boldsymbol{M}^{a}(\ell), \boldsymbol{M}^{o}(\ell)\right\rangle$ using (3)-(5) with $\ell(\theta)=\mathbb{1}_{\left[\theta>\theta_{1}\right]} x\left(t_{1}\right)$. The market composition $\Psi_{2}$ yields a new market tightness $t_{2}$ and a new cutoff $\theta_{2}=\bar{\theta}\left(\Psi_{2}\right)$. From $\theta_{2}$ and $t_{2}$ we get another market composition $\Psi_{3}$, and so on. An equilibrium is a fixed point of such an iterative process.

Proposition A.1 For any $x \in \mathcal{X}$, there is a unique random search SME given by $\left(\theta_{r}^{*}, \Psi_{r}^{*}, t_{r}^{*}\right)$. The market tightness satisfies

$$
t_{r}^{*}=1+\left(\frac{k_{a}-k_{o}}{k_{o}}\right)\left[\frac{\gamma+(1-\gamma) x\left(t_{r}^{*}\right)}{\gamma+(1-\gamma) x\left(t_{r}^{*}\right) F\left(\theta_{r}^{*}\right)}\right],
$$

and the cutoff $\theta_{r}^{*}$ satisfies the indifference condition

$$
\theta_{r}^{*}=(1-\gamma)\left(\frac{x\left(t_{r}^{*}\right)}{\gamma+x\left(t_{r}^{*}\right)(1-\gamma)}\right) \frac{k_{o}}{k_{a}} \int_{\theta_{r}^{*}}^{1} \omega d F(\omega) .
$$

Proof. Let $\left(\theta_{r}, \Psi_{r}, t_{r}\right)$ be a random search SME. By definition, $\Psi_{r}=\left\langle\boldsymbol{G}(\cdot ; \ell), \boldsymbol{M}^{a}(\ell), \boldsymbol{M}^{o}(\ell)\right\rangle$ for $\ell(\theta)=x\left(t_{r}\right) \mathbb{1}_{\left[\theta>\theta_{r}\right]}$ for all $\theta \in \Theta$. As market tightness must be consistent with the market ${ }^{21} \mathbb{1}_{[\cdot]}$ is the indicator function. 
composition, $t_{r}=\frac{\boldsymbol{M}^{a}(\ell)}{\boldsymbol{M}^{o}(\ell)}$. Substituting in (4) and (5) for $\boldsymbol{M}^{o}(\ell)$ and $\boldsymbol{M}^{a}(\ell)$ yields (A1). It is clear that $t_{r}<T_{r}$ for some large but finite $T_{r}$.

Taking $\Psi_{r}$ as a given, the agents use the cutoff strategy $\bar{\theta}\left(\Psi_{r}\right)$. The value of random search can then be rewritten as

$$
U^{r}\left(\Psi_{r}\right)=\frac{x\left(t_{r}\right)}{t_{r} \gamma+(1-\gamma)\left(1-\boldsymbol{G}\left(\bar{\theta}\left(\Psi_{r}\right) ; \ell\right)\right) x\left(t_{r}\right)} \int_{\bar{\theta}\left(\Psi_{r}\right)}^{1} \omega d \boldsymbol{G}(\omega ; \ell)
$$

By definition, $\theta_{r}=\bar{\theta}\left(\Psi_{r}\right)=(1-\gamma) U^{r}\left(\Psi_{r}\right)$. Substituting in (3) and (A1) for $\boldsymbol{G}(\cdot ; \ell)$ and $t_{r}$ respectively yields (A2).

We now have a pair of fixed point problems. Let $\mathcal{R}: \Theta \times\left[0, T_{r}\right] \rightarrow \Theta \times\left[0, T_{r}\right]$ with $\mathcal{R}(\theta, t)$ given by the pair

$$
(\underbrace{\left(\frac{(1-\gamma) x(t)}{x(t)(1-\gamma)+\gamma}\right) \frac{k_{o}}{k_{a}} \int_{\theta}^{1} \omega d F(\omega)}_{=\mathcal{R}_{1}(\theta, t)}, \underbrace{1+\left(\frac{k_{a}-k_{o}}{k_{o}}\right)\left[\frac{\gamma+(1-\gamma) x(t)}{\gamma+(1-\gamma) x(t) F(\theta)}\right]}_{\mathcal{R}_{2}(\theta, t)})
$$

For $\left(\theta_{r}, \Psi_{r}, t_{r}\right)$ to be a random search SME, $\left(\theta_{r}, t_{r}\right)$ must be the fixed point of $\mathcal{R}$. Notice that $\mathcal{R}$ is a continuous mapping from a compact space into itself. By Brouwer's fixed point theorem, there is at least one fixed point, which proves existence of a random SME for any $x \in \mathcal{X}$.

In order to prove uniqueness, I first make two remarks. First, $\mathcal{R}_{1}(\cdot, t)$ is decreasing in $\theta$ with $\mathcal{R}_{1}(0, t) \geq 0$ and $\mathcal{R}_{1}(1, t)=0<1$ for all $t \in\left[0, T_{r}\right]$. Thus, $\mathcal{R}_{1}(\cdot, t)$ has a unique fixed point in $\Theta$ for all $t \in\left[0, T_{r}\right]$. Additionally, $\mathcal{R}_{1}(\theta, \cdot)$ is increasing in $t$ for all $\theta \in \Theta$ which implies that the unique fixed point of $\mathcal{R}_{1}(\cdot, t)$ is increasing in $t$. Second, $\mathcal{R}_{2}(\cdot, t)$ is decreasing in $\theta$ for all $t \in\left[0, T_{r}\right]$, and $\mathcal{R}_{2}(\theta, \cdot)$ is increasing and concave in $t$ for all $\theta \in \Theta$.

Assume that $\left(\theta_{r}^{1}, t_{r}^{1}\right)$ and $\left(\theta_{r}^{2}, t_{r}^{2}\right)$ are both fixed points of $\mathcal{R}$. In particular, $\theta_{r}^{i}$ is the unique fixed point of $\mathcal{R}_{1}\left(\cdot ; t_{r}^{i}\right)$ and $t_{r}^{i}$ is a fixed point of $\mathcal{R}_{2}\left(\theta_{r}^{i} ; \cdot\right)$ for $i=1,2$. If $t_{r}^{1} \geq t_{r}^{2}$, then $\theta_{r}^{1} \geq \theta_{r}^{2}$. For some $\pi \in[0,1], t_{r}^{2}=\pi t_{r}^{1}$. By the monotonicity and concavity of $\mathcal{R}_{2}$, we have

$$
t_{r}^{2}=\mathcal{R}_{2}\left(\theta_{r}^{2}, t_{r}^{2}\right) \geq \mathcal{R}_{2}\left(\theta_{r}^{1}, t_{r}^{2}\right)=\mathcal{R}_{2}\left(\theta_{r}^{1}, \pi t_{r}^{1}\right) \geq \underbrace{\pi \mathcal{R}_{2}\left(\theta_{r}^{1}, t_{r}^{1}\right)}_{\pi t_{r}^{1}=t_{r}^{2}}+(1-\pi) \underbrace{\mathcal{R}_{2}\left(\theta_{r}^{1}, 0\right)}_{>0}
$$


which would lead to a contradiction unless $\pi=1$, i.e., $t_{r}^{1}=t_{r}^{2}$. But that implies $\theta_{r}^{1}=\theta_{r}^{2}$. In other words, there is a unique fixed point $\left(\theta_{r}^{*}, t_{r}^{*}\right)$ of $\mathcal{R}$.

\section{A.1.2. Frictional Directed Search Protocol}

Under a directed search protocol, we need to consider the market tightness of each sub-market $\{S(\theta)\}_{\theta \in \Theta}$. Given a market composition $\Psi=\left\langle G, M^{a}, M^{o}\right\rangle$ and a queueing strategy $Q$ with both $G$ and $Q$ absolutely continuous, the market tightness of a sub-market $S(\theta)$ is given by $t(\theta)=M^{a} q(\theta) / M^{o} g(\theta) .^{22}$

Given a pairing technology $x \in \mathcal{X}$, an agent in sub-market $S(\theta)$ gets matched with probability $\alpha(\theta ; Q, \Psi)=x(t(\theta)) / t(\theta)$ with the convention that $x(0) / 0=\lim _{t \rightarrow 0} x(t) / t=1$. Similarly, an object of type $\theta$ gets matched to an agent with probability $\beta(\theta ; Q, \Psi)=x(t(\theta))$. The value of directed search in sub-market $S(\theta)$ is still given by

$$
\begin{gathered}
U^{d}(\theta ; Q, \Psi)=\alpha(\theta ; Q, \Psi) \theta+(1-\gamma)(1-\alpha(\theta ; Q, \Psi)) U^{d}(\theta ; Q, \Psi) \\
=\frac{\alpha(\theta ; Q, \Psi) \theta}{\gamma+\alpha(\theta ; Q, \Psi)(1-\gamma)} .
\end{gathered}
$$

Definition A.2 A directed search $S M E$ is given by the tuple $\left(Q_{d}, \Psi_{d}, t_{d}\right)$, where $t_{d}: \Theta \rightarrow \mathbb{R}_{+}$ is the market tightness function, such that

(i) $Q_{d}(\theta)=\int_{0}^{\theta} q_{d}(\omega) d \omega$ for all $\theta \in \Theta$ with $Q_{d}(1)=1$,

(ii) $q_{d}(\theta)>0$ implies $\theta \in \arg \max _{\theta^{\prime} \in \Theta} U^{d}\left(\theta^{\prime} ; Q_{d}, \Psi_{d}\right)$,

(iii) $\Psi_{d}=\left\langle\boldsymbol{G}(\cdot ; \ell), \boldsymbol{M}^{a}(\ell), \boldsymbol{M}^{o}(\ell)\right\rangle$ as given by $(3)-(5)$ with $\ell(\cdot)=x\left(t_{d}(\cdot)\right)$, and

(iv) $t_{d}(\theta)=\frac{\boldsymbol{M}^{a}(\ell) q_{d}(\theta)}{\boldsymbol{M}^{\circ}(\ell) \boldsymbol{g}(\theta ; \ell)}$ for almost all $\theta \in \Theta$.

To get some intuition for Definition A.2, consider the following iterative process: Take some arbitrary $\Psi_{1}$ and $Q_{1}$, and compute the market tightness function $t_{1}(\cdot)$. Using the matching probabilities $x\left(t_{1}(\theta)\right) / t_{1}(\theta)$ for each $S(\theta)$, the agents optimally choose which sub-markets to enter. Aggregating their choices gives a new queueing $\operatorname{CDF} Q_{2}$. We also derive a new market composition $\Psi_{2}=\left\langle\boldsymbol{G}(\cdot ; \ell), \boldsymbol{M}^{a}(\ell), \boldsymbol{M}^{o}(\ell)\right\rangle$ using (3)-(5) with $\ell(\theta)=x\left(t_{1}(\theta)\right)$. The market composition $\Psi_{2}$ and the queueing $\operatorname{CDF} Q_{2}$ yield a new market tightness function $t_{2}(\cdot)$, which

\footnotetext{
${ }^{22}$ From Lemma 1, a steady state market distribution $G$ has a density function $g$ that is bounded below almost everywhere by $\gamma \underline{f}>0$. Hence, it is WLOG to consider only distributions with a positive density so that $t(\theta)$ is well-defined everywhere.
} 
then leads to a new queueing $\operatorname{CDF} Q_{3}$ and a new market composition $\Psi_{3}$, and so on. An equilibrium is a fixed point of such an iterative process.

\section{Proposition A.2}

For any $x \in \mathcal{X}$, there is a unique directed search $S M E$ given by $\left(Q_{d}^{*}, \Psi_{d}^{*}, t_{d}^{*}\right)$ where

(i) $\Psi_{d}^{*}=\left\langle\boldsymbol{G}(\cdot ; \ell), \boldsymbol{M}^{a}(\ell), \boldsymbol{M}^{o}(\ell)\right\rangle$ as given by $(3)-(5)$ with $\ell(\cdot)=x\left(t_{d}^{*}(\cdot)\right)$,

(ii) $Q_{d}^{*}\left(\theta_{d}^{*}\right)=0$, and

(iii) $U^{d}\left(\theta ; Q_{d}^{*}, \Psi_{d}^{*}\right)=\left\{\begin{array}{cc}\theta & \text { for } \theta \leq \theta_{d}^{*} \\ \theta_{d}^{*} & \text { for } \theta \geq \theta_{d}^{*}\end{array}\right.$.

The market tightness function satisfies

$$
t_{d}^{*}(\theta)=\left\{\begin{array}{cc}
x\left(t_{d}^{*}(\theta)\right)\left(\frac{\theta-(1-\gamma) \theta_{d}^{*}}{\gamma \theta_{d}^{*}}\right)>0 & \text { if } \theta>\theta_{d}^{*} \\
0 & \text { if } \theta<\theta_{d}^{*}
\end{array}\right.
$$

and the cutoff $\theta_{d}^{*} \in \Theta$ satisfies the indifference condition

$$
\theta_{d}^{*}=\frac{k_{o}}{k_{a}} \int_{\theta_{d}^{*}}^{1} \omega \frac{x\left(t_{d}^{*}(\omega)\right)}{\gamma+(1-\gamma) x\left(t_{d}^{*}(\omega)\right)} d F(\omega) .
$$

Proof. Let $\left(Q_{d}, \Psi_{d}, t_{d}\right)$ be a directed search SME. By Lemma 2 (which holds for any $x \in \mathcal{X}$ ), there exists a cutoff $\theta_{d} \in(0,1)$ such that $q_{d}(\theta)>(=) 0$ for all $\theta>(<) \theta_{d}$. By definition, $t_{d}(\theta)>(=) 0$ for all $\theta>(<) \theta_{d}$. Furthermore, $U^{d}\left(\theta ; Q_{d}, \Psi_{d}\right)=\theta_{d}$ for all $\theta \geq \theta_{d}$.

For all $\theta>\theta_{d}, U^{d}\left(\theta ; Q_{d}, \Psi_{d}\right)=\theta_{d}$ necessitates that $\alpha\left(\theta ; Q_{d}, \Psi_{d}\right)=\frac{\gamma \theta_{d}}{\theta-(1-\gamma) \theta_{d}}$. By definition, $\alpha\left(\theta ; Q_{d}, \Psi_{d}\right)=\frac{x\left(t_{d}(\theta)\right)}{t_{d}(\theta)}$. Equating these two expressions for the agent match probability yields (A3).

Define $\mathcal{D}\left(\cdot ; \theta, \theta_{d}\right): \mathbb{R}_{+} \rightarrow \mathbb{R}_{+}$by

$$
\mathcal{D}\left(t ; \theta, \theta_{d}\right)=x(t)\left(\frac{\theta-(1-\gamma) \theta_{d}}{\gamma \theta_{d}}\right) \mathbb{1}_{\left[\theta>\theta_{d}\right]},
$$


For all $\theta \in \Theta$ and all $\theta_{d} \in(0,1), \mathcal{D}$ is continuous, (weakly) increasing, and (weakly) concave in $t$. Furthermore, $\mathcal{D}\left(T_{d} ; \theta, \theta_{d}\right)<T_{d}$ for some large but finite $T_{d}$ because $\lim _{t \rightarrow \infty} x(t)=1 .{ }^{23}$

$\mathcal{D}\left(\cdot ; \theta, \theta_{d}\right)$ has one trivial fixed point with $0=\mathcal{D}\left(0 ; \theta, \theta_{d}\right)$. However, market tightness must be positive for $\theta>\theta_{d}$. Thus, we must show that there is a positive fixed point. Given the properties of $\mathcal{D}\left(\cdot ; \theta, \theta_{d}\right)$, there are only three cases to consider:

1. 0 is the unique fixed point of $\mathcal{D}\left(\cdot ; \theta, \theta_{d}\right)$,

2. There is an interval $\left[0, t^{\prime}\right]$ such that each $t \in\left[0, t^{\prime}\right]$ is a fixed point of $\mathcal{D}\left(\cdot ; \theta, \theta_{d}\right)$, and

3. There is a unique $t^{\prime}>0$ such that $\left\{0, t^{\prime}\right\}$ are the only fixed points of $\mathcal{D}\left(\cdot ; \theta, \theta_{d}\right)$.

Figure 3a, Figure 3b, and Figure 3c depict Cases 1,2, and 3 respectively. Lemma A.1 shows that $\mathcal{D}\left(\cdot ; \theta, \theta_{d}\right)$ has a unique positive fixed point for all $\theta_{d} \in(0,1)$ and all $\theta>\theta_{d}$ by ruling out Cases 1 and 2 .

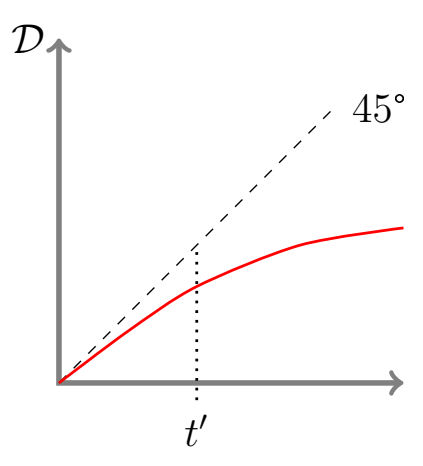

(a) Case 1

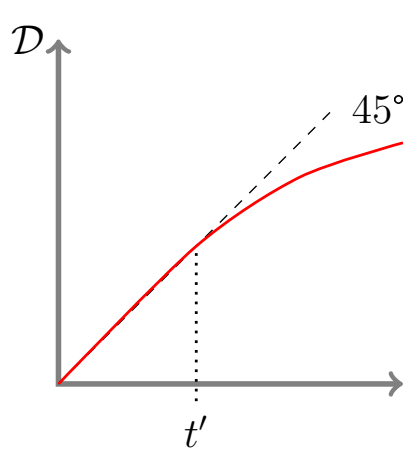

(b) Case 2

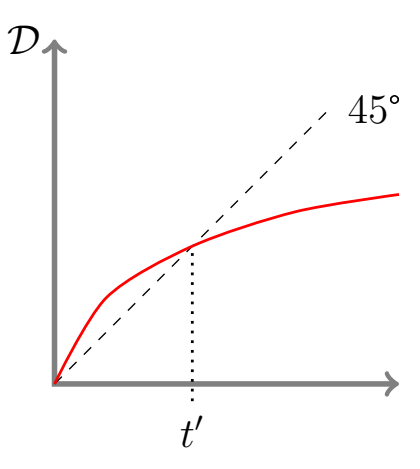

(c) Case 3

Figure 3

Lemma A.1 For any given $\theta_{d} \in(0,1)$ and any $\theta>\theta_{d}$,

A.1.1. There is a unique positive fixed point $\boldsymbol{t}\left(\theta, \theta_{d}\right)$ of $\mathcal{D}\left(\cdot ; \theta, \theta_{d}\right)$, and

\section{A.1.2. For any $\theta>\theta_{d}^{\prime}>\theta_{d}, \boldsymbol{t}\left(\theta, \theta_{d}\right) \geq \boldsymbol{t}\left(\theta, \theta_{d}^{\prime}\right)$.}

Additionally, for any positive and monotone sequence $\theta>\theta_{d}^{1}>\theta_{d}^{2}>\ldots$ converging to 0 , $\lim _{\theta_{d}^{n} \rightarrow 0} \boldsymbol{t}\left(\theta, \theta_{d}^{n}\right)=\infty$.

\footnotetext{
${ }^{23} T_{d}$ depends on $\theta_{d}$. In particular, it suffices to take $T_{d}>\frac{1-(1-\gamma) \theta_{d}}{\gamma \theta_{d}}$.
} 


\section{Proof.}

(A.1.1.) Consider an arbitrary $\theta>\theta_{d}>0$. Let $\underline{\nabla} \mathcal{D}\left(\cdot ; \theta, \theta_{d}\right)$ be the infimum of the subderivatives of $\mathcal{D}\left(\cdot ; \theta, \theta_{d}\right)$ given by

$$
\underline{\nabla} \mathcal{D}\left(t ; \theta, \theta_{d}\right)=\lim _{\epsilon \rightarrow 0} \frac{\mathcal{D}\left(t+\epsilon ; \theta, \theta_{d}\right)-\mathcal{D}\left(t ; \theta, \theta_{d}\right)}{\epsilon} .
$$

Suppose either Case 1 or Case 2 hold which implies $\mathcal{D}\left(t ; \theta, \theta_{d}\right) \leq t$ for all $t>0$. By concavity,

$$
\mathcal{D}\left(0 ; \theta, \theta_{d}\right) \leq \mathcal{D}\left(t ; \theta, \theta_{d}\right)+\underline{\nabla} \mathcal{D}\left(t ; \theta, \theta_{d}\right)(0-t) \Longrightarrow \underline{\nabla} \mathcal{D}\left(t ; \theta, \theta_{d}\right) t \leq \mathcal{D}\left(t ; \theta, \theta_{d}\right) \leq t .
$$

Thus, for all $t>0, \underline{\nabla} \mathcal{D}\left(t ; \theta, \theta_{d}\right) \leq 1$. Since $\mathcal{D}\left(\cdot ; \theta, \theta_{d}\right)$ is concave in $t, \underline{\nabla} \mathcal{D}\left(\cdot ; \theta, \theta_{d}\right)$ is lower semi-continuous in $t$ and $\underline{\nabla \mathcal{D}}\left(0 ; \theta, \theta_{d}\right) \leq \liminf _{t \rightarrow 0} \underline{\nabla \mathcal{D}}\left(t ; \theta, \theta_{d}\right) \leq 1$. However, $\underline{\nabla} \mathcal{D}\left(0 ; \theta, \theta_{d}\right)$ is well-defined and given by

$$
\underline{\nabla} \mathcal{D}\left(0 ; \theta, \theta_{d}\right)=\underbrace{\lim _{\epsilon \rightarrow 0} \frac{x(\epsilon)}{\epsilon}}_{=1}\left(\frac{\theta-(1-\gamma) \theta_{d}}{\gamma \theta_{d}}\right)>1
$$

One of the three cases must hold but Case 1 and 2 were just ruled out. Thus, there exists a unique positive fixed point $\boldsymbol{t}\left(\theta, \theta_{d}\right)$ of $\mathcal{D}\left(\cdot ; \theta, \theta_{d}\right)$ for all $\theta_{d} \in(0,1)$ and all $\theta>\theta_{d}$.

(A.1.2) $\mathcal{D}\left(t ; \theta, \theta_{d}\right)$ is increasing in $t$ and decreasing in $\theta_{d}$. For any $\theta>\theta_{d}^{\prime}>\theta_{d}$,

$$
\boldsymbol{t}\left(\theta, \theta_{d}^{\prime}\right)=\mathcal{D}\left(\boldsymbol{t}\left(\theta, \theta_{d}^{\prime}\right) ; \theta, \theta_{d}^{\prime}\right) \leq \mathcal{D}\left(\boldsymbol{t}\left(\theta, \theta_{d}^{\prime}\right) ; \theta, \theta_{d}\right)
$$

As $\mathcal{D}$ is concave and increasing in $t, \boldsymbol{t}\left(\theta, \theta_{d}\right)=\mathcal{D}\left(\boldsymbol{t}\left(\theta, \theta_{d}\right) ; \theta, \theta_{d}\right)$ implies $\boldsymbol{t}\left(\theta, \theta_{d}^{\prime}\right) \leq \boldsymbol{t}\left(\theta, \theta_{d}\right)$.

Finally, for any positive and monotone sequence $\theta>\theta_{d}^{1}>\theta_{d}^{2}>\ldots>0$,

$$
\frac{x\left(\left(\boldsymbol{t}\left(\theta, \theta_{d}^{n}\right)\right)\right.}{\boldsymbol{t}\left(\theta, \theta_{d}^{n}\right)}=\frac{\gamma \theta_{d}^{n}}{\theta-(1-\gamma) \theta_{d}^{n}}, \quad \forall n \geq 1 .
$$

If $\theta_{d}^{n} \rightarrow 0$, the RHS also converges to 0 . Since $x(t) / t$ is decreasing but always positive for any finite $t$, we need $\lim _{\theta_{d}^{n} \rightarrow 0} \boldsymbol{t}\left(\theta, \theta_{d}^{n}\right)=\infty$ for the LHS to also converge to 0 .

For any $\theta \in \Theta$ and $\theta_{d} \in(0,1)$ let $t\left(\theta ; \theta_{d}\right)=\mathbb{1}_{\left[\theta>\theta_{d}\right]} \boldsymbol{t}\left(\theta, \theta_{d}\right)$. Clearly, $t\left(\theta ; \theta_{d}\right)$ is a fixed point of $\mathcal{D}\left(\cdot ; \theta, \theta_{d}\right)$ and selects the unique positive fixed point whenever $\theta>\theta_{d}$. 
Lemma A.2 For any sequence $\left\{\theta_{d}^{n}\right\}_{n=1}^{\infty}$ such that $\theta_{d}^{n} \in(0,1)$ for all $n$ and $\theta_{d}^{n}$ converges to $\theta_{d} \in(0,1), t\left(\cdot ; \theta_{d}^{n}\right)$ converges pointwise almost everywhere to $t\left(\cdot ; \theta_{d}\right)$.

Proof. For any $\theta \neq \theta_{d}$, I show that for $t\left(\theta ; \theta_{d}^{n}\right)$ converges to $t\left(\theta ; \theta_{d}\right)$.

Take some $\theta<\theta_{d}$. There exists an $N$ large enough (which depends on $\theta$ ) such that for all $n>N, \theta_{d}^{n}>\theta$ and $t\left(\theta ; \theta_{d}^{n}\right)=0=t\left(\theta ; \theta_{d}\right)$.

Take some $\theta>\theta_{d}$. The sequence $\left\{\theta_{d}^{n}\right\}_{n=1}^{\infty}$ has a monotone sub-sequence $\left\{\theta_{d}^{n_{m}}\right\}_{n_{m}=1}^{\infty}$ such that either $\theta_{d}^{n_{m}} \uparrow \theta_{d}$ or $\theta_{d}^{n_{m}} \downarrow \theta_{d}$. Again, there exists a large enough $N$ (depends on $\theta$ ) such that for all $n_{m} \geq N, \theta>\theta_{d}^{n_{m}}$.

Let $C\left(\theta, \theta_{d}^{n_{m}}\right)$ be the correspondence mapping $\left(\theta, \theta_{d}^{n_{m}}\right)$ into the fixed points of $\mathcal{D}\left(\cdot ; \theta, \theta_{d}^{n_{m}}\right)$. By Lemma A.1.1, $C\left(\theta, \theta_{d}^{n_{m}}\right)=\left\{0, t\left(\theta ; \theta_{d}^{n_{m}}\right)\right\}$ for all $n_{m} \geq N$. Additionally, $\mathcal{D}\left(\cdot ; \theta, \theta_{d}^{n_{m}}\right)$ converges to $\mathcal{D}\left(\cdot ; \theta, \theta_{d}\right)$ uniformly in $t$. Hence, $C\left(\theta, \theta_{d}\right)$ is upper hemicontinuous in a neighborhood of $\theta_{d}$, i.e., $\lim _{n_{m} \rightarrow \infty} t\left(\theta ; \theta_{d}^{n_{m}}\right) \in C\left(\theta, \theta_{d}\right)=\left\{0, t\left(\theta ; \theta_{d}\right)\right\} .{ }^{24}$

If $\theta_{d}^{n_{m}} \uparrow \theta_{d}$, then for $n_{m} \geq N, t\left(\theta ; \theta_{d}^{n_{m}}\right) \geq t\left(\theta ; \theta_{d}^{n_{m+1}}\right) \geq \ldots \geq t\left(\theta ; \theta_{d}\right)>0$ because $t(\theta ; \cdot)$ is decreasing (Lemma A.1.2). Hence, $\inf _{n_{m}} t\left(\theta, \theta_{d}^{n_{m}}\right)>0$ which implies $\lim _{n_{m} \rightarrow \infty} t\left(\theta ; \theta_{d}^{n_{m}}\right)=t\left(\theta ; \theta_{d}\right)$.

Similarly, if $\theta_{d}^{n_{m}} \downarrow \theta_{d}$, then for all $n_{m} \geq N, 0<t\left(\theta ; \theta_{d}^{n_{m}}\right) \leq t\left(\theta ; \theta_{d}^{n_{m+1}}\right) \leq \ldots \leq t\left(\theta ; \theta_{d}\right)$. Hence, $\sup _{n_{m}} t\left(\theta ; \theta_{d}^{n_{m}}\right)>0$ which implies $\lim _{n_{m} \rightarrow \infty} t\left(\theta ; \theta_{d}^{n_{m}}\right)=t\left(\theta ; \theta_{d}\right)$.

As the market tightness function must also be consistent with the equilibrium market composition,

$$
\underbrace{\frac{\boldsymbol{M}^{a}(\ell) q_{d}(\theta)}{\boldsymbol{M}^{o}(\ell) \boldsymbol{g}(\theta ; \ell)}}_{=t\left(\theta ; \theta_{d}\right)}=x\left(t\left(\theta ; \theta_{d}\right)\right)\left(\frac{\theta-(1-\gamma) \theta_{d}}{\gamma \theta_{d}}\right), \quad \forall \theta>\theta_{d}>0,
$$

$\overline{{ }^{24} \text { Given } \theta>\theta_{d}>0 \text {, take } \epsilon>0 \text { such that }}\{0, \theta\} \nsubseteq N_{\epsilon}\left(\theta_{d}\right)$. The mapping from $\left(t, \tilde{\theta}_{d}\right) \in\left[0, T_{d}\right] \times N_{\epsilon}\left(\theta_{d}\right) \mapsto$ $-\left(t-\mathcal{D}\left(t ; \theta, \tilde{\theta}_{d}\right)\right)^{2}$ is continuous in $\left(t, \tilde{\theta}_{d}\right)$. Then, $C\left(\theta, \tilde{\theta}_{d}\right)=\arg \max _{t \in\left[0, T_{d}\right]}-\left(t-\mathcal{D}\left(t ; \theta, \tilde{\theta}_{d}\right)\right)^{2}$ is upper hemicontinuous in $\tilde{\theta}_{d} \in N_{\epsilon}\left(\theta_{d}\right)$ by the Maximum Theorem. 
where $\ell(\theta)=x\left(t\left(\theta ; \theta_{d}\right)\right)$. Rearranging the equality and integrating over the interval $\left[\theta_{d}, 1\right]$ gives

$$
\theta_{d}[\gamma \boldsymbol{M}^{a}(\ell) \underbrace{\int_{\theta_{d}}^{1} d Q_{d}(\omega)}_{=1}+(1-\gamma) \boldsymbol{M}^{o}(\ell) \int_{\theta_{d}}^{1} x\left(t_{d}(\omega)\right) d G_{d}(\omega)]=\boldsymbol{M}^{o}(\ell) \int_{\theta_{d}}^{1} \omega x\left(t_{d}(\omega)\right) d G_{d}(\omega) .
$$

Using (3)-(5) with $\ell(\theta)=x\left(t\left(\theta ; \theta_{d}\right)\right)$ simplifies the expression to

$$
\theta_{d}=\underbrace{\frac{k_{o}}{k_{a}} \int_{\theta_{d}}^{1} \omega \frac{x\left(t\left(\omega ; \theta_{d}\right)\right)}{\gamma+(1-\gamma) x\left(t\left(\omega ; \theta_{d}\right)\right)} d F(\omega)}_{\triangleq \mathbb{D}\left(\theta_{d}\right)}
$$

From Lemma A.1, $\mathbb{D}(\cdot)$ is a decreasing function on $(0,1)$. From Lemma A.2 and the dominated convergence theorem, $\mathbb{D}(\cdot)$ is also continuous. We can continuously extend $\mathbb{D}$ onto $\Theta \triangleq[0,1]$ by setting $\mathbb{D}(1)=\lim _{\theta_{d} \rightarrow 1} \mathbb{D}\left(\theta_{d}\right)=0<1$ and

$$
\mathbb{D}(0)=\lim _{\theta_{d} \rightarrow 0} \mathbb{D}\left(\theta_{d}\right)=\frac{k_{o}}{k_{a}} \int_{\Theta} \omega \lim _{\theta_{d} \rightarrow 0} \frac{x\left(\boldsymbol{t}\left(\omega, \theta_{d}\right)\right)}{\gamma+(1-\gamma) x\left(\boldsymbol{t}\left(\omega, \theta_{d}\right)\right)} d F(\omega)=\frac{k_{o}}{k_{a}} \mathbb{E}_{F}[\theta]>0
$$

where the last equality follows from Lemma A.1 and the assumption that $\lim _{t \rightarrow \infty} x(t)=1$. Therefore, there is a unique positive fixed point $\theta_{d}^{*}$ to $\mathbb{D}(\cdot)$, and $t_{d}^{*}(\theta)=t\left(\theta ; \theta_{d}^{*}\right)$ for all $\theta \in \Theta$.

\section{A.1.3. Social Welfare Comparison with Frictional Search}

In general, it is difficult to establish which search protocol dominates in terms of social welfare for any $x \in \mathcal{X}$. However, it is possible to show that Theorem 1 continues to hold when the pairing technology is not too frictional.

Consider a sequence of paring technologies $\left\{x_{n}\right\}_{n=1}^{\infty}$ with $x_{n} \in \mathcal{X}$. Given a pairing technology $x_{n}$ and a search cost $\gamma \in(0,1]$, let $\theta_{r}^{n}(\gamma)$ and $\theta_{d}^{n}(\gamma)$ be the SME cutoffs under a random and directed search protocol as characterized by Proposition A.1 and Proposition A.2. Define social welfare under the two protocols by $\boldsymbol{W}_{\gamma, n}^{r}=k_{a} \frac{\theta_{r}^{n}(\gamma)}{1-\gamma}$ and $\boldsymbol{W}_{\gamma, n}^{d}=k_{a} \theta_{d}^{n}(\gamma)$.

Let $\bar{x}(t)=\min \{t, 1\}$ denote the frictionless pairing technology. By assumption, $x(\cdot) \leq \bar{x}(\cdot)$ for all $x \in \mathcal{X}$. Recall that Proposition 1 and Proposition 2 characterize the SME cutoffs $\theta_{r}^{*}(\gamma)$ and $\theta_{d}^{*}(\gamma)$ under the random and directed search protocol for $\bar{x}$ and a search cost $\gamma \in(0,1]$. The social welfare under the two protocols are given by $\boldsymbol{W}_{\gamma}^{r}=k_{a} \frac{\theta_{r}^{*}(\gamma)}{1-\gamma}$ and $\boldsymbol{W}_{\gamma}^{d}=k_{a} \theta_{d}^{*}(\gamma)$.

Theorem A.1 Fix $\gamma>0$. Consider a sequence of paring technologies $\left\{x_{n}\right\}_{n=1}^{\infty}$ in $\mathcal{X}$ and let 
$\boldsymbol{W}_{\gamma, n}^{i}$ be the social welfare under protocol $i=r, d$. If $x_{n}$ converges uniformly to $\bar{x}$, there exists a sub-sequence $\left\{x_{n_{k}}\right\}_{n_{k}=1}^{\infty}$ and a large enough $N$ such that $\boldsymbol{W}_{\gamma, n_{k}}^{r}>\boldsymbol{W}_{\gamma, n_{k}}^{d}$ for all $n_{k}>N$.

The proof is an application of standard lower hemi-continuity arguments and is skipped. Briefly, if $x_{n}$ converges uniformly to $\bar{x}$, then there is a sub-sequence of pairing technologies $\left\{x_{n_{k}}\right\}_{n_{k}}$ such that the associated welfare $\boldsymbol{W}_{\gamma, n_{k}}^{i}$ converges to $\boldsymbol{W}_{\gamma}^{i}$ for $i=r, d$. From Theorem $1, \boldsymbol{W}_{\gamma}^{r}>\boldsymbol{W}_{\gamma}^{d}$ for all $\gamma>0$, and the result follows.

\section{A.2. Non-Scarcity}

In this section, I consider the robustness of the welfare result of Theorem 1 to a search market without scarcity. Fix the in-flowing mass of agents at some $k_{a}>0$, and let $k_{o}=\lambda k_{a}$ for some constant $\lambda>0$. The basic model assumed scarcity, i.e., $\lambda \leq 1$. The scarcity assumption no longer holds when $\lambda>1$.

\section{A.2.1. Random Search Protocol Without Scarcity}

Regardless of $\lambda$, an object is matched with probability $x(t)=\min \{t, 1\}$ whereas an agent is matched with probability $x(t) / t$, where $t=M^{a} / M^{o}$ is the market tightness. We can therefore directly apply the results in Proposition A.1.

Proposition A.3 For all $\lambda>0$, there is a unique random search SME given by $\left(\theta_{r}^{*}, \Psi_{r}^{*}, t_{r}^{*}\right)$. The market tightness satisfies

$$
t_{r}^{*}=1+\left(\frac{1-\lambda}{\lambda}\right)\left[\frac{\gamma+(1-\gamma) \min \left\{t_{r}^{*}, 1\right\}}{\gamma+(1-\gamma) \min \left\{t_{r}^{*}, 1\right\} F\left(\theta_{r}^{*}\right)}\right]
$$

and the cutoff $\theta_{r}^{*}$ satisfies the indifference condition

$$
\theta_{r}^{*}=(1-\gamma)\left(\frac{\min \left\{t_{r}^{*}, 1\right\}}{\gamma+\min \left\{t_{r}^{*}, 1\right\}(1-\gamma)}\right) \lambda \int_{\theta_{r}^{*}}^{1} \omega d F(\omega)
$$

The market composition $\Psi_{r}^{*}=\left\langle\boldsymbol{G}(\cdot ; \ell), \boldsymbol{M}^{a}(\ell), \boldsymbol{M}^{o}(\ell)\right\rangle$ is derived from (3)-(5) with $\ell(\theta)=$ $\mathbb{1}_{\left[\theta>\theta_{r}^{*}\right]} \min \left\{t_{r}^{*}, 1\right\}$ for all $\theta \in \Theta$.

The proof is precisely the same as that of Proposition A.1 and is therefore skipped. To see how this connects to Proposition 1 , notice from (A5) that $t_{r}^{*} \geq 1$ when $\lambda \leq 1$. Thus, $\min \left\{t_{r}^{*}, 1\right\}=1$ and (A6) simplifies to (9). 


\section{A.2.2. Directed Search Protocol Without Scarcity}

The scarcity assumption did not play any role in the analysis of the directed search protocol in Section 1.4. Hence, the characterization of a directed search SME in Proposition 2 holds for any $\lambda>0$.

\section{Proposition A.4}

For all $\lambda>0$, there is a unique directed search $S M E$, and it is given by $\left(Q_{d}^{*}, \Psi_{d}^{*}\right)$ where

(i) $\Psi_{d}^{*}=\left\langle\boldsymbol{G}\left(\cdot ; \theta_{d}^{*}\right), \boldsymbol{M}^{a}\left(\theta_{d}^{*}\right), \boldsymbol{M}^{o}\left(\theta_{d}^{*}\right)\right\rangle$ as given in $(6)-(8)$,

(ii) $Q_{d}^{*}(\theta)=\left\{\begin{array}{cc}0 & \text { if } \quad \theta \leq \theta_{d}^{*} \\ \int_{\theta_{d}^{*}}^{\theta} \frac{\lambda\left(\omega-(1-\gamma) \theta_{d}^{*}\right)}{\left(1-\lambda(1-\gamma)\left(1-F\left(\theta_{d}^{*}\right)\right)\right) \theta_{d}^{*}} d F(\omega) & \text { for } \quad \theta \geq \theta_{d}^{*}\end{array}\right.$.

The cutoff $\theta_{d}^{*} \in \Theta$ satisfies the indifference condition

$$
\theta_{d}^{*}=\lambda \int_{\theta_{d}^{*}}^{1} \omega d F(\omega)
$$

\section{A.2.3. Social Welfare Comparison}

For a given $(\lambda, \gamma) \in \mathbb{R}_{>0} \times(0,1]$ and an absolutely continuous $F$, let $\theta_{r}^{*}(\lambda, \gamma, F)$ be the random search SME cutoff given by (A6). Social welfare under the random search protocol is given by $\boldsymbol{W}^{r}(\lambda, \gamma, F)=k_{a} \frac{\theta_{r}^{*}(\lambda, \gamma, F)}{1-\gamma}$. Similarly, let $\theta_{d}^{*}(\lambda, \gamma, F)$ be the directed search SME cutoffs given by (A7). Define $\boldsymbol{W}^{d}(\lambda, \gamma, F)=k_{a} \theta_{d}^{*}(\lambda, \gamma, F)$ as the social welfare under the directed search protocol.

Theorem A.2 For any $\gamma \in(0,1]$ and any absolutely continuous distribution $F$, there exist cutoffs $1<\underline{\lambda}(\gamma, F) \leq \bar{\lambda}(\gamma, F)<\infty$ such that

i. $\boldsymbol{W}^{d}(\lambda, \gamma, F) \leq \boldsymbol{W}^{r}(\lambda, \gamma, F)$ for all $\lambda \leq \underline{\lambda}(\gamma, F)$, and

ii. $\boldsymbol{W}^{d}(\lambda, \gamma, F) \geq \boldsymbol{W}^{r}(\lambda, \gamma, F)$ for all $\lambda \geq \bar{\lambda}(\gamma, F)$.

Proof. From Theorem $1, \boldsymbol{W}^{d}(\lambda, \gamma, F)<\boldsymbol{W}^{r}(\lambda, \gamma, F)$ for all $\gamma \in(0,1]$ and all absolutely continuous $F$ when $\lambda \leq 1$. I will therefore focus on the case where $\lambda>1$. By (A5), $t_{r}^{*}<1$. 
From Lemma 1, any steady state market composition $\Psi=\left\langle G, M^{a}, M^{o}\right\rangle$ satisfies $M^{a} \geq k_{a}$ and $M^{o}=M^{a}+\gamma^{-1}\left(k_{o}-k_{a}\right)=M^{a}+\gamma^{-1} k_{a}(\lambda-1)$. Thus, any steady state market tightness satisfies

$$
t=\frac{M^{a}}{M^{o}}=\frac{M^{a}}{M^{a}+\gamma^{-1} k_{a}(\lambda-1)} \geq \frac{\gamma}{\gamma+\lambda-1} \triangleq \underline{t} .
$$

Define the mapping $\mathcal{P}(\cdot ; t): \Theta \rightarrow \mathbb{R}_{+}$by

$$
\mathcal{P}(\theta ; t)=\left(\frac{t}{\gamma+t(1-\gamma)}\right) \lambda \int_{\theta}^{1} \omega d F(\omega)
$$

for $0<t<1$. The mapping $\mathcal{P}(\cdot ; t)$ is strictly decreasing in $\theta$ for all $t \in(0,1)$ with $\mathcal{P}(0 ; t)>0$ and $\mathcal{P}(1 ; t)=0<1$; hence, $\mathcal{P}(\cdot ; t)$ has a unique fixed point in $\Theta$. Additionally, $\mathcal{P}(\theta ; \cdot)$ is increasing in $t$ for all $\theta \in \Theta$. Thus, the fixed point of $\mathcal{P}(\cdot ; t)$ is increasing in $t$.

Notice that for all $\theta \in \Theta$,

$$
\mathcal{P}\left(\theta ; t_{r}^{*}\right) \geq \mathcal{P}(\theta ; \underline{t})=\int_{\theta}^{1} \omega d F(\omega)
$$

From Proposition A.3, $\theta_{r}^{*}(\lambda, \gamma, F)$ is the unique fixed point of $(1-\gamma) \mathcal{P}\left(\cdot ; t_{r}^{*}\right)$ while $\theta_{r}^{*}(1, \gamma, F)$ is the unique fixed point of $(1-\gamma) \mathcal{P}(\cdot ; \underline{t})$. Moreover, $\theta_{r}^{*}(\lambda, \gamma, F) \geq \theta_{r}^{*}(1, \gamma, F)$ because $t_{r}^{*} \geq \underline{t}$.

Define

$$
\underline{\lambda}(\gamma, F)=\sup \left\{\lambda: \theta_{d}^{*}(\lambda, \gamma, F) \leq \frac{\theta_{r}^{*}(1, \gamma, F)}{1-\gamma}\right\} .
$$

$\theta_{d}^{*}(\lambda, \gamma, F)$ is a continuous and strictly increasing function of $\lambda$, and $\theta_{d}^{*}(\lambda, \gamma, F)<\frac{\theta_{r}^{*}(\lambda, \gamma, F)}{1-\gamma}$ for all $\lambda \in(0,1]$ by Theorem 1 . Hence, $\underline{\lambda}(\gamma, F)>1$. Additionally, for all $1<\lambda \leq \underline{\lambda}(\gamma, F)$,

$$
\boldsymbol{W}^{d}(\lambda, \gamma, F)=k_{a} \theta_{d}^{*}(\lambda, \gamma, F) \leq k_{a} \frac{\theta_{r}^{*}(1, \gamma, F)}{1-\gamma} \leq k_{a} \frac{\theta_{r}^{*}(\lambda, \gamma, F)}{1-\gamma}=\boldsymbol{W}^{r}(\lambda, \gamma, F)
$$

which proves the first result.

To prove the second result, consider the single-agent problem under the random search protocol. Let $U_{S A}^{r}$ be the payoff under the single-agent problem given by

$$
U_{S A}^{r}=\int_{\Theta} \max \left\{\theta,(1-\gamma) U_{S A}^{r}\right\} d F(\omega)
$$


and let $\theta_{r}^{S A}(\gamma, F)=(1-\gamma) U_{S A}^{r}$ be the unique indifference cutoff. The single-agent payoff is an upper bound of the equilibrium payoff in a random search market, i.e., $\theta_{r}^{S A}(\gamma, F) \geq \theta_{r}^{*}(\lambda, \gamma, F)$ for all finite $\lambda .^{25}$

Define

$$
\bar{\lambda}(\gamma, F)=\inf \left\{\lambda: \theta_{d}^{*}(\lambda, \gamma, F) \geq \frac{\theta_{r}^{S A}(\gamma, F)}{1-\gamma}\right\}
$$

Recall that $\theta_{d}^{*}(\lambda, \gamma, F)$ is a continuous and strictly increasing function of $\lambda$. Additionally, $\lim _{\lambda \rightarrow \infty} \theta_{d}^{*}(\lambda, \gamma, F)=1$ which is the outcome for a single-agent directed search problem. On the other hand, the outcome for a single-agent random search problem is given by

$$
U_{S A}^{r}=\frac{\theta_{r}^{S A}(\gamma, F)}{1-\gamma}=\frac{1}{1-(1-\gamma) F\left(\theta_{r}^{S A}(\gamma, F)\right)} \int_{\theta_{r}^{S A}(\gamma, F)}^{1} \omega d F(\omega) \leq \mathbb{E}_{F}\left[\theta \mid \theta \geq \theta_{r}^{S A}(\gamma, F)\right]<1
$$

which implies $\bar{\lambda}(\gamma, F)<\infty$. Finally, for all $\lambda>\bar{\lambda}(\gamma, F)$,

$$
\boldsymbol{W}^{d}(\lambda, \gamma, F)=k_{a} \theta_{d}^{*}(\lambda, \gamma, F) \geq k_{a} \frac{\theta_{r}^{S A}(\gamma, F)}{1-\gamma} \geq k_{a} \frac{\theta_{r}^{*}(\lambda, \gamma, F)}{1-\gamma}=\boldsymbol{W}^{r}(\lambda, \gamma, F)
$$

which proves the second result.

\section{A.3. Hybrid Search with Coarse Information}

I have considered two search protocols so far: random search in which the agents cannot observe the quality of an object prior to pairing with it, and directed search in which the agents have full information about an object's quality ex-ante. In this section, I consider a market which is divided into a finite number of sub-markets that provide the agents with coarse ex-ante information about quality. The agents use a hybrid search protocol in which they first direct their search to one of the sub-markets and then randomly sample the objects within their chosen sub-market. The main result of this section is to show that social welfare and equilibrium payoffs are lower under a hybrid search protocol than under a random search protocol.

Let $\left\{S_{k}\right\}_{k=1}^{n}$ denote a monotone $n$-partition of $\Theta$ such that $S_{1}=\left[x_{0}, x_{1}\right), \ldots, S_{n}=\left[x_{n-1}, x_{n}\right]$ with $0=x_{0}<x_{1}<\ldots<x_{n}=1 .^{26}$ In what follows, I refer to $S_{k}$ as sub-market $k$. In a hybrid search protocol, each agent first chooses one of the $n$ sub-markets to enter and then searches

\footnotetext{
${ }^{25}$ It is straightforward to show that $\theta_{r}^{S A}(\gamma, F)=\lim _{\lambda \rightarrow \infty} \theta_{r}^{*}(\lambda, \gamma, F)$.

${ }^{26}$ For $n=1$, I adopt the convention that $S_{1}=[0,1]$.
} 
using a random search protocol within the chosen sub-market. ${ }^{27}$

Let $F_{k}=F\left(x_{k}\right)-F\left(x_{k-1}\right)$ for $k=1, \ldots n$. Given a market composition $\Psi=\left\langle G, M^{a}, M^{o}\right\rangle$, similarly define $G_{k}=G\left(x_{k}\right)-G\left(x_{k-1}\right)$. Let $M_{k}^{a}$ denote the mass of objects and agents in $S_{k}$ so that $\sum_{k=1}^{n} M_{k}^{a}=M^{a}$.

Let $\alpha_{k}=\min \left\{\frac{M^{o} G_{k}}{M_{k}^{a}}, 1\right\}$ and $\beta_{k}=\min \left\{\frac{M_{k}^{a}}{M^{\circ} G_{k}}, 1\right\}$ denote the agent and the object pairing probabilities respectively in $S_{k}$. Denote the agent entry probabilities by $\zeta \triangleq\left\{\zeta_{k}\right\}_{k=1}^{n}$ with $\sum_{k=1}^{n} \zeta_{k}=1$ where $\zeta_{k} \geq 0$ is the probability that a newly arrived agent chooses sub-market $S_{k}$. I refer to $S_{k}$ as an on-path sub-market if $k \in S u p p(\boldsymbol{\zeta}) \triangleq\left\{k^{\prime}: \zeta_{k^{\prime}}>0\right\}$ and as off-path if $k \notin \operatorname{Supp}(\boldsymbol{\zeta})$.

Under a hybrid search protocol, an agent's value of entering an on-path sub-market $S_{k}$ is given by

$$
U_{k}^{n}=\alpha_{k} \int_{S_{k}} \max \left\{\theta,(1-\gamma) U_{k}^{n}\right\} \frac{d G(\theta)}{G_{k}}+\left(1-\alpha_{k}\right)(1-\gamma) U_{k}^{n} .
$$

In equilibrium, an agent who enters an on-path sub-market $S_{k}$ uses a cutoff strategy $\bar{\theta}_{k} \in S_{k}$ such that she matches with any object in $S_{k}$ with quality $\theta>\bar{\theta}_{k}$ and rejects any object with quality $\theta<\bar{\theta}_{k}$. The cutoff is determined by the indifference condition

$$
\bar{\theta}_{k} \geq(1-\gamma) U_{k}^{n}
$$

with equality if $\bar{\theta}_{k}>\inf S_{k}=x_{k-1}$. Additionally, the agents must be indifferent across all on-path sub-markets, i.e., $U_{k}^{n}=U_{k^{\prime}}^{n} \triangleq \boldsymbol{U}^{n}$ for all $k, k^{\prime} \in \operatorname{Supp}(\boldsymbol{\zeta})$.

For each $k \in \operatorname{Supp}(\boldsymbol{\zeta})$ and each $\theta \in S_{k}$, the on-path inflow-outflow dynamics for objects must satisfy

$$
\begin{array}{r}
M^{o}\left(G(\theta)-G\left(x_{k-1}\right)\right) \gamma=k_{o}\left(F(\theta)-F\left(x_{k-1}\right)\right), \forall \theta<\bar{\theta}_{k}, \\
M^{o}\left(G(\theta)-G\left(\bar{\theta}_{k}\right)\right)\left(\gamma+\beta_{k}(1-\gamma)\right)=k_{o}\left(F(\theta)-F\left(\bar{\theta}_{k}\right)\right), \forall \theta>\bar{\theta}_{k},
\end{array}
$$

\footnotetext{
${ }^{27}$ In particular, the agents and objects in each $S_{k}$ are randomly paired to each other. Upon pairing with an object, an agent observes the object's true quality. She then chooses to either match with the object and exit, or to reject the object and search again. The in-flow and out-flow of agents and objects is the same as the basic model. Once again, it is without loss of generality to assume that an agent remains in the first sub-market she enters. The agents must be indifferent among all on-path sub-markets. As their payoffs are stationary, it does not matter if they remain in the first on-path sub-market they enter or if they randomize
} 
while the on-path inflow-outflow dynamics for the agents is given by

$$
M_{k}^{a}\left(\gamma+\alpha_{k}(1-\gamma)\left(\frac{G\left(x_{k}\right)-G\left(\bar{\theta}_{k}\right)}{G_{k}}\right)\right)=k_{a} \zeta_{k}
$$

For an off-path sub-market $S_{k}, M_{k}^{a}=0$ which implies that $\alpha_{k}=1$, and $\beta_{k}=0$. As objects in $S_{k}$ leave the market only via death, the inflow-outflow dynamics satisfy

$$
M^{o}\left(G(\theta)-G\left(x_{k-1}\right)\right) \gamma=k_{o}\left(F(\theta)-F\left(x_{k-1}\right)\right), \forall \theta \in S_{k}
$$

An agent's payoff from a one-shot deviation into an off-path sub-market $S_{k}$ is given by

$$
U_{k}^{n}=\int_{S_{k}} \max \left\{\theta,(1-\gamma) \boldsymbol{U}^{n}\right\} \frac{d G(\theta)}{G_{k}}=\int_{S_{k}} \max \left\{\theta,(1-\gamma) \boldsymbol{U}^{n}\right\} \frac{d F(\theta)}{F_{k}},
$$

where the second equality follows from (A12).

Definition A.3 Given a monotone n-partition $\left\{S_{k}\right\}_{k=1}^{n}$, a hybrid search $S M E$ is given by a market composition $\Psi=\left\langle G, M^{a}, M^{o}\right\rangle$, entry probabilities $\boldsymbol{\zeta} \triangleq\left\{\zeta_{k}\right\}_{k=1}^{n}$, and cutoffs $\left\{\bar{\theta}_{k}\right\}_{k \in \operatorname{Supp}(\boldsymbol{\zeta})}$ such that

(i) For all $k, k^{\prime} \in \operatorname{Supp}(\boldsymbol{\zeta}), U_{k}^{n}=U_{k^{\prime}}^{n} \triangleq \boldsymbol{U}^{n}$ as given by (A8),

(ii) For all $k \in \operatorname{Supp}(\boldsymbol{\zeta}), \bar{\theta}_{k} \in S_{k}$ and satisfies $\left(I C_{k}\right)$ with equality if $\bar{\theta}_{k}>\inf S_{k}$,

(iii) For all $k \notin \operatorname{Supp}(\boldsymbol{\zeta}), U_{k}^{n}$ is given by (A13) and $\boldsymbol{U}^{n} \geq U_{k}^{n}$, and

(iv) The market composition is derived from (A9)-(A12).

The first two conditions describe the on-path hybrid search SME outcomes: Taking the market composition as a given, $(i)$ each agent must be indifferent among all on-path sub-markets, and $(i i)$ the cutoff strategy an agent uses in an on-path sub-market is sequentially rational. Condition ( iii) states that the agents cannot be better off by taking a one-shot deviation to any off-path sub-market. Finally, condition $(i v)$ states that the market composition must be consistent with the agents' entry probabilities and cutoff strategies.

The following lemma simplifies the characterization of a hybrid search SME.

Lemma A.3 For any hybrid search SME, there exits a threshold $k^{*} \in\{1,2, \ldots, n\}$ such that $\zeta_{k}>0$ if, and only if, $k \geq k^{*}$. Additionally, if $k^{*}<n$, then $\bar{\theta}_{k}=\inf S_{k}=x_{k-1}$ and $\beta_{k}=1$ for all $k>k^{*}$. 
Proof. Suppose in some hybrid search SME, $\zeta_{k}>0=\zeta_{k^{\prime}}$ for some $k<k^{\prime} \leq n$. The on-path equilibrium payoff $U_{k}^{n}$ is given by (A8) and satisfies

$$
U_{k}^{n} \leq \int_{S_{k}} \max \left\{\theta,(1-\gamma) U_{k}^{n}\right\} \frac{d G(\theta)}{G_{k}} \leq \int_{S_{k}} \max \left\{\theta, \bar{\theta}_{k}\right\} \frac{d G(\theta)}{G_{k}} \leq x_{k}=\sup S_{k}
$$

As $S_{k^{\prime}}$ is an off-path sub-market, $U_{k^{\prime}}^{n}$ is given by (A13). Since the partition is monotone, $U_{k^{\prime}}^{n}=\mathbb{E}_{F}\left[\theta \mid \theta \in S_{k^{\prime}}\right]>\inf S_{k^{\prime}} \geq \sup S_{k} \geq U_{k}^{n}$, which yields a profitable deviation. Hence, there exists some $k^{*}$ such that $\zeta_{k}>0$ if, and only if, $k \geq k^{*}$.

Now suppose that $k^{*}<n$ and consider some $k>k^{*}$. Then $\bar{\theta}_{k}>\bar{\theta}_{k^{*}} \geq(1-\gamma) \boldsymbol{U}^{n}$. From $\left(I C_{k}\right)$, $\bar{\theta}_{k} \neq(1-\gamma) \boldsymbol{U}^{n}$ only if $\bar{\theta}_{k}=\inf S_{k}=x_{k-1}$. Finally, a necessary condition for $U_{k}^{n}=U_{k^{*}}^{n}$ is that $\alpha_{k}<1$ which implies (by definition) $\alpha_{k}=\frac{M^{o} G_{k}}{M_{k}^{a}}$ and $\beta_{k}=1$.

Theorem A.3 Social welfare and equilibrium payoffs are higher under a random search protocol than under any hybrid search protocol with a monotone $n$-partition of $\Theta$.

Proof. For any hybrid search SME, there exists a $k^{*}$ by Lemma A.3 such that agents enter only $\left\{S_{k}\right\}_{k \geq k^{*}}$. For any $k>k^{*}$ (if $\left.k^{*}<n\right), \bar{\theta}_{k}=x_{k-1}, \alpha_{k}=\frac{M^{o} G_{k}}{M_{k}^{a}}$, and $\beta_{k}=1$. Then, (A8) can be rewritten as

$$
\begin{aligned}
U_{k}^{n} & =\frac{\alpha_{k}}{\gamma+(1-\gamma) \alpha_{k}} \int_{S_{k}} \omega \frac{d G(\omega)}{G_{k}} \\
& =\frac{k_{o}}{k_{a} \zeta_{k}} \int_{S_{k}} \omega d F(\omega)
\end{aligned}
$$

where the second equality follows from simplifying (A10) and (A11) to

$$
\begin{aligned}
& M^{o}\left(G(\theta)-G\left(x_{k-1}\right)\right)=k_{o}\left(F(\theta)-F\left(x_{k-1}\right)\right), \forall \theta \in S_{k}, \\
& M_{k}^{a}\left(\gamma+\alpha_{k}(1-\gamma)\right)=k_{a} \zeta_{k} .
\end{aligned}
$$

For $S_{k^{*}},(\mathrm{~A} 8)$ can be rewritten as

$$
U_{k^{*}}^{n}=\frac{\alpha_{k^{*}}}{\gamma+(1-\gamma) \alpha_{k^{*}}\left(\frac{G\left(x_{k^{*}}\right)-G\left(\bar{\theta}_{k^{*}}\right)}{G_{k^{*}}}\right)} \int_{\bar{\theta}_{k^{*}}}^{x_{k^{*}}} \omega \frac{d G(\omega)}{G_{k^{*}}}
$$

From $(\mathrm{A} 11)$, we can express $\gamma+(1-\gamma) \alpha_{k^{*}}\left(\frac{G\left(x_{k^{*}}\right)-G\left(\bar{\theta}_{k^{*}}\right)}{G_{k^{*}}}\right)$ as $\frac{k_{a} \zeta_{k^{*}}}{M_{k^{*}}^{a}}$. Furthermore, by definition 
of the pairing probabilities, $\alpha_{k^{*}}=\beta_{k^{*}} \frac{M^{o} G_{k^{*}}}{M_{k^{*}}^{a}}$. Finally, from $(\mathrm{A} 10)$, we get $M^{o}\left(G(\theta)-G\left(\bar{\theta}_{k^{*}}\right)\right)=$ $k_{o}\left(F(\theta)-F\left(\bar{\theta}_{k^{*}}\right)\right)\left(\gamma+\beta_{k^{*}}(1-\gamma)\right)^{-1}$ for all $\theta>\bar{\theta}_{k^{*}}$. Substituting in these expressions into $U_{k^{*}}^{n}$ yields

$$
U_{k^{*}}^{n}=\frac{k_{o}}{k_{a} \zeta_{k^{*}}}\left(\frac{\beta_{k^{*}}}{\gamma+(1-\gamma) \beta_{k^{*}}}\right) \int_{\bar{\theta}_{k^{*}}}^{x_{k^{*}}} \omega d F(\omega)
$$

Since agents must be indifferent among all the on-path sub-markets, the equilibrium payoffs must satisfy $U_{k}^{n}=U_{k^{*}}^{n}=\boldsymbol{U}^{n}$ for all $k \geq k^{*}$. Additionally, $\sum_{k=k^{*}}^{n} \zeta_{k}=1$, and

$$
\begin{aligned}
\boldsymbol{U}^{n} & =\sum_{k=k^{*}}^{n} \zeta_{k} U_{k}^{n} \\
& =\frac{k_{o}}{k_{a}}\left(\frac{\beta_{k^{*}}}{\gamma+(1-\gamma) \beta_{k^{*}}} \int_{\bar{\theta}_{k^{*}}}^{x_{k^{*}}} \omega d F(\omega)+\int_{x_{k^{*}}}^{1} \omega d F(\omega)\right) \\
& \leq \frac{k_{o}}{k_{a}} \int_{\bar{\theta}_{k^{*}}}^{1} \omega d F(\omega) \\
& =\mathbb{U}\left(\bar{\theta}_{k^{*}}\right)
\end{aligned}
$$

where $\mathbb{U}(x)=\int_{x}^{1} \omega d F$. From Proposition 1, the unique equilibrium payoff under random search $\mathrm{SME}$ is given by $\boldsymbol{U}^{r}=\frac{\theta_{r}^{*}}{1-\gamma}=\mathbb{U}\left(\theta_{r}^{*}\right)$.

Suppose $\boldsymbol{U}^{n}>\boldsymbol{U}^{r}$. As $\mathbb{U}(\cdot)$ is a strictly decreasing function, it must be that $\bar{\theta}_{k^{*}}<\theta_{r}^{*}$. However, this would imply

$$
\boldsymbol{U}^{r}=\frac{\theta_{r}^{*}}{1-\gamma}>\frac{\bar{\theta}_{k^{*}}}{1-\gamma} \geq \boldsymbol{U}^{n}
$$

where the last inequality follows from $\left(I C_{k^{*}}\right)$. Therefore, in any hybrid search SME, $\boldsymbol{U}^{n} \leq \boldsymbol{U}^{r}$. As social welfare is just a re-scaling of the equilibrium payoffs by $k_{a}$, this concludes the proof.

Remark A.1 Notice that Theorem A.3 does not claim existence of a hybrid search SME. In fact, there are $n$-partitions of $\Theta$ for which an equilibrium does not exist as there are too many (in)equalities that need to be satisfied simultaneously. First, agents must be indifferent across all on-path sub-markets with $U_{k^{*}}^{n}=U_{k^{*}+1}^{n}=\ldots=U_{n}^{n}$. Second, the cutoff $\bar{\theta}_{k^{*}}$ is linked to $U_{k^{*}}^{n}$ (and therefore all $U_{k}^{n}$ for $k \geq k^{*}$ ) through $\left(I C_{k^{*}}\right)$. Finally, the agents must choose to not deviate down with $U_{k^{*}}^{n} \geq U_{k^{*}-1}^{n}$. Nonetheless, the possible non-existence of such equilibrium outcomes 
does not invalidate the general negative result of the theorem.

\section{A.4. Taste Heterogeneity}

In this section, I generalize the basic model so that objects are both horizontally and vertically differentiated, and agents have heterogeneous tastes. I consider three different search protocols: a random search protocol in which agents have no information, a partially directed search protocol in which the agents have information only about the horizontal attributes of objects, and a directed search protocol in which the agents have full information about both the objects' horizontal attributes and quality.

Objects are horizontally differentiated based on two horizontal attribute types denoted by $j=1,2$. Each attribute $j$ is further vertically differentiated by quality with $\theta_{j} \in \Theta_{j} \triangleq[0,1]$. There are also two types of agents denoted by $i=1,2$. As in the basic model, there are $k_{a}$ new agents and $k_{o}$ new objects that arrive in each period. A fraction $\pi \in(0,1)$ of the incoming agents and objects are type 1 . Additionally, the quality of a newly arrived object of attribute $j$ is distributed according to an absolutely continuous distribution function $F_{j}$.

A type $i$ agent's ex-post payoff from matching with a type $\theta_{j} \in \Theta_{j}$ object is given by $u_{i}\left(\theta_{j}\right)=\theta_{j} \mathbb{1}_{[i=j]}$. In other words, a type 1 agent is only compatible with a type 1 object and a type 2 agent is only compatible with a type 2 object. The assumption is stark but it allows for a simple generalization of the results so far, Importantly, it also provides the best setting for directed search to facilitate assortative matching and thus "stacks the deck" against random search protocols.

\section{A.4.1. Random Search Protocol}

Under a random search protocol, the agents get no information about quality or the horizontal attribute. Let $\Psi_{1}=\left\langle G_{1}, M_{1}^{a}, M_{1}^{o}\right\rangle$ be the market composition of the type 1 agents and objects, and similarly define $\Psi_{2}$. Let $M^{a}=M_{1}^{a}+M_{2}^{a}$ and $M^{o}=M_{1}^{o}+M_{2}^{o}$. Each object of type $j$ is paired to a compatible agent with probability $M_{j}^{a} / M^{a}$ while each agent of type $i$ is paired to a compatible object with probability $M_{i}^{o} / M^{a}$. 
The value of random search for a type $i$ agent is given by

$$
\begin{aligned}
U_{i}^{r} & =\frac{M_{i}^{o}}{M^{a}} \int_{\Theta_{i}} \max \left\{\omega_{i},(1-\gamma) U_{i}^{r}\right\} d G_{i}\left(\omega_{i}\right)+\left(1-\frac{M_{i}^{o}}{M^{a}}\right)(1-\gamma) U_{i}^{r} \\
& =\frac{M_{i}^{o}}{M^{a} \gamma+M_{i}^{o}(1-\gamma)\left(1-G_{i}\left(\bar{\theta}_{i}\right)\right)} \int_{\bar{\theta}_{i}}^{1} \omega_{i} d G_{i}\left(\omega_{i}\right),
\end{aligned}
$$

where $\bar{\theta}_{i}$ is a type $i$ agent's optimal cutoff derived from the indifference condition $\bar{\theta}_{i}=(1-\gamma) U_{i}^{r}$.

The inflow-outflow conditions for type $i$ agents is given

$$
M_{i}^{a}\left(\frac{M^{a} \gamma+M_{i}^{o}(1-\gamma)\left(1-G_{i}\left(\bar{\theta}_{i}\right)\right)}{M^{a}}\right)=k_{a} \pi_{i}
$$

where, with some abuse of notation, $\pi_{i}$ is the fraction of new type $i$ agents. Similarly, the inflow-outflow conditions for type $j$ objects is given by

$$
\begin{gathered}
M_{j}^{o} G_{j}\left(\theta_{j}\right) \gamma=k_{o} \pi_{j} F_{j}\left(\theta_{i}\right), \forall \theta_{j}<\bar{\theta}_{j} \\
M_{j}^{o}\left(G_{j}\left(\theta_{j}\right)-G_{j}\left(\bar{\theta}_{j}\right)\right)\left(\frac{M_{j}^{a}+\gamma M_{-j}^{a}}{M^{a}}\right)=k_{o} \pi_{j}\left(F_{j}\left(\theta_{j}\right)-F_{j}\left(\bar{\theta}_{j}\right)\right), \forall \theta_{j}>\bar{\theta}_{j}
\end{gathered}
$$

where, again with some abuse of notation, $\pi_{j}$ is the fraction of new type $j$ objects. Using (A14) and (A16), the value of random search simplifies to

$$
U_{i}^{r}=\frac{k_{o}}{k_{a}}\left(\frac{M_{i}^{a}}{M_{i}^{a}+\gamma M_{-i}^{a}}\right) \int_{\bar{\theta}_{i}}^{1} \omega_{i} d F_{i}\left(\omega_{i}\right)
$$

and the mass of type $i$ agents simplifies to

$$
M_{i}^{a}=\frac{k_{a} \pi_{i}\left(M_{i}^{a}+\gamma M_{-i}^{a}\right)}{\gamma M_{i}^{a}+\gamma^{2} M_{-i}^{a}+(1-\gamma) k_{o} \pi_{i}\left(1-F_{i}\left(\bar{\theta}_{i}\right)\right)} .
$$

Let $\mathbb{T}: \prod_{i=1}^{2} \Theta_{i} \times\left[k_{a} \pi_{i}, \frac{k_{a} \pi_{i}}{\gamma}\right] \rightarrow \prod_{i=1}^{2} \Theta_{i} \times\left[k_{a} \pi_{i}, \frac{k_{a} \pi_{i}}{\gamma}\right]$ be the function that maps the tuple $\left(\bar{\theta}_{i}, M_{i}^{a}\right)_{i=1,2}$ into $\mathbb{T}\left(\left(\bar{\theta}_{i}, M_{i}^{a}\right)_{i=1,2}\right)$ given by

$$
\left((1-\gamma) \frac{k_{o}}{k_{a}}\left(\frac{M_{i}^{a}}{M_{i}^{a}+\gamma M_{-i}^{a}}\right) \int_{\bar{\theta}_{i}}^{1} \omega_{i} d F_{i}\left(\omega_{i}\right), \frac{k_{a} \pi_{i}\left(M_{i}^{a}+\gamma M_{-i}^{a}\right)}{\gamma M_{i}^{a}+\gamma^{2} M_{-i}^{a}+(1-\gamma) k_{o} \pi_{i}\left(1-F_{i}\left(\bar{\theta}_{i}\right)\right)}\right)_{i=1,2} .
$$


An equilibrium outcome in a random search SME is given by a fixed point of $\mathbb{T}(\cdot)$ which exists as $\mathbb{T}(\cdot)$ is a continuous mapping from a compact space into itself. Given $\pi \in(0,1)$, social welfare is given by $^{28}$

$$
\mathcal{W}_{\pi}^{r}=\left\{\sum_{i=1,2} k_{a} \pi_{i} \frac{\theta_{i, r}^{*}}{1-\gamma}:\left(\theta_{i, r}^{*}, M_{i, r}^{a *}\right)_{i=1,2}=\mathbb{T}\left(\left(\theta_{i, r}^{*}, M_{i, r}^{a *}\right)_{i=1,2}\right)\right\} .
$$

\section{A.4.2. Partially Directed Search Protocol}

Under a partially directed search protocol, the agents get full information about the objects' horizontal attribute but get no information about quality. The search market is divided into two sub-markets $\left\{\mathcal{S}_{j}\right\}_{j=1,2}$ so that sub-market $\mathcal{S}_{j}$ contains only objects of type $j$. The agents can choose which sub-market to enter but observe the quality $\theta_{j}$ of an object only after pairing to it.

The agents derive a positive utility only if they match with a compatible object. Therefore, each type $i$ agent will enter $\mathcal{S}_{j}$ if, and only, if $j=i$. In other words, the sub-markets allow for perfect assortative matching based on attribute types. Furthermore, the strategic choices of the agents in sub-market $\mathcal{S}_{j}$ imposes no externalities on sub-market $\mathcal{S}_{-j}$ because the agents perfectly sort themselves into the two sub-markets.

Within each sub-market $\mathcal{S}_{j}$, the agents use a random search protocol. In each period, a mass of $k_{a} \pi_{j}$ new agents and $k_{o} \pi_{j}$ new objects arrive into $\mathcal{S}_{j}$ where the new objects' quality distribution is given by $F_{j}$. Except for a parametric change in the exogenous variables, the search problem in $\mathcal{S}_{j}$ is strategically equivalent to the random search protocol in the basic model. I can thus directly apply the result from Proposition 1.

In a partially directed search protocol, a type $i$ agent enters sub-market $\mathcal{S}_{i}$, matches with all objects of quality $\theta_{i}>\theta_{i, p}^{*}$ (subscript $p$ for partially directed), and rejects all objects of quality $\theta_{i}<\theta_{i, p}^{*}$. The cutoff is characterized by the unique solution to

$$
\theta_{i, p}^{*}=(1-\gamma) \int_{\theta_{i, p}^{*}}^{1} \omega_{i} d F_{i}\left(\omega_{i}\right)
$$

and social welfare is given by

$$
\boldsymbol{W}_{\pi}^{p}=\sum_{i=1,2} k_{a} \pi_{i} \frac{\theta_{i, p}^{*}}{1-\gamma} .
$$

${ }^{28}$ The formulation accommodates for the possibility of multiple SME outcomes. The uniqueness or multiplicity of SME's is tangential to the purposes of this section. 


\section{A.4.3. Directed Search Protocol}

Under a directed search protocol, the agents get full information about both the objects' horizontal attribute and quality. As in the partially directed search protocol, the search market is divided into two sub-markets $\left\{\mathcal{S}_{j}\right\}_{j=1,2}$ so that sub-market $\mathcal{S}_{j}$ contains only objects of type $j$. Furthermore, each sub-market $\mathcal{S}_{j}$ is further divided into sub-sub-markets $\left\{S_{j}\left(\theta_{j}\right)\right\}_{\theta_{j} \in \Theta_{j}}$. The agents can choose in which sub-sub-market to search.

Once again, each type $i$ agent will enter $\mathcal{S}_{j}$ if, and only, if $j=i$. Within each sub-market $\mathcal{S}_{j}$, the agents use a directed search protocol. Except for a parametric change in the exogenous variables, the search problem in $\mathcal{S}_{j}$ is strategically equivalent to the directed search protocol in the basic model. I can thus directly apply the result from Proposition 2.

In a directed search protocol, type $i$ agents enter sub-market $\mathcal{S}_{i}$ and queue only at sub-submarkets $\left\{S_{i}\left(\theta_{i}\right)\right\}_{\theta_{i} \geq \theta_{i, d}^{*}}$. The cutoff is characterized by the unique solution to

$$
\theta_{i, d}^{*}=\int_{\theta_{i, p}^{*}}^{1} \omega_{i} d F_{i}\left(\omega_{i}\right)
$$

and social welfare is given by

$$
\boldsymbol{W}_{\pi}^{d}=\sum_{i=1,2} k_{a} \pi_{i} \frac{\theta_{i, d}^{*}}{1-\gamma}
$$

\section{A.4.4. Social Welfare Comparison with Taste Heterogeneity}

This section extends the intuition of Theorem 1 to the case with horizontal differentiation and heterogeneous tastes. In the current simple model, providing information about the horizontal attributes facilitates assortative matching between compatible agents and objects without inducing congestion. On the other hand, information about quality leads to congestion externalities as in the basic model.

Under a directed search protocol, there is a welfare gain due to the assortative matching but a welfare loss due to congestion. Which of these two forces dominates depends on the parameters. However, the following result shows that when the level of heterogeneity is small, social welfare would be higher if the agents had no information (random search protocol) than if they had full information (directed search protocol). Yet, social welfare is always unambiguously higher when the agents only get information about the horizontal attributes (partially directed search protocol).

Theorem A.4 For all $\pi \in(0,1), \boldsymbol{W}_{\pi}^{p}>\boldsymbol{W}_{\pi}^{d}$, and $\boldsymbol{W}_{\pi}^{p}>\boldsymbol{W}_{\pi}^{r}$ for all $\boldsymbol{W}_{\pi}^{r} \in \mathcal{W}_{\pi}^{r}$. Furthermore, 
$\boldsymbol{W}_{\pi}^{r}>\boldsymbol{W}_{\pi}^{d}$ for all $\boldsymbol{W}_{\pi}^{r} \in \mathcal{W}_{\pi}^{r}$ whenever $\pi(1-\pi)$ is small enough.

Proof. The proof for $\boldsymbol{W}_{\pi}^{p}>\boldsymbol{W}_{\pi}^{d}$ immediately follows from the same argument as the proof of Theorem 1.

Let $\left(\theta_{i, r}^{*}, M_{i, r}^{a *}\right)_{i=1,2}$ be a random search SME outcome. Taking $\left\{M_{i, r}^{a *}\right\}_{i=1,2}$ as a given, the cutoff $\theta_{i, r}^{*}$ is the unique fixed point of the function

$$
(1-\gamma) \frac{k_{o}}{k_{a}} \underbrace{\left(\frac{M_{i, r}^{a *}}{M_{i, r}^{a *}+\gamma M_{-i, r}^{a *}}\right)}_{<1} \mathbb{U}_{i}(\cdot)
$$

where $\mathbb{U}_{i}\left(\theta_{i}\right)=\int_{\theta_{i}}^{1} \omega_{i} d F_{i}$. On the other hand, the cutoff $\theta_{i, p}^{*}$ under a partially directed search protocol is the unique fixed point of $(1-\gamma) \frac{k_{o}}{k_{a}} \mathbb{U}_{i}(\cdot)$ as given by (A19). Because $\mathbb{U}_{i}$ is a decreasing function, $\theta_{i, r}^{*}<\theta_{i, p}^{*}$. As the choice of the random search SME was arbitrary,

$$
\boldsymbol{W}_{\pi}^{r}=\sum_{i=1,2} k_{a} \pi_{i} \frac{\theta_{i, r}^{*}}{1-\gamma}<\boldsymbol{W}_{\pi}^{p}=\sum_{i=1,2} k_{a} \pi_{i} \frac{\theta_{i, p}^{*}}{1-\gamma}
$$

for all $\boldsymbol{W}_{\pi}^{r} \in \mathcal{W}_{\pi}^{r}$.

Additionally, from (A18), any random search SME satisfies $M_{i, r}^{a *} \in\left[k_{a} \pi_{i}, \frac{k_{a} \pi_{i}}{\gamma}\right]$ for $i=1,2$. As $\pi$ approaches $1, M_{2, r}^{a *}$ approaches 0 which implies $\frac{M_{1, r}^{a *}}{M_{1, r}^{a *}+\gamma M_{2, r}^{a *}}$ approaches 1 . In the limit, the gap between $\theta_{1, r}^{*}$ and $\theta_{1, p}^{*}$ vanishes. However, $\frac{\theta_{i, p}^{*}}{1-\gamma}>\theta_{i, d}^{*}$ for all $i=1,2$ and all $\pi \in[0,1]$. Hence, $\frac{\theta_{1, r}^{*}}{1-\gamma}>\theta_{1, d}^{*}$ when $\pi$ is close to 1 . As social welfare is primarily determined by the welfare of type 1 agents when $\pi$ is close to 1 , we also have $\boldsymbol{W}_{\pi}^{r}>\boldsymbol{W}_{\pi}^{d}$ for all $\boldsymbol{W}_{\pi}^{r} \in \mathcal{W}_{\pi}^{r}$. The same argument establishes the welfare ranking across the three protocols when $\pi$ is close to 0 as welfare is primarily determined by the payoff of type 2 agents.

\section{B. Search with Ex-post Transfers: Relaxing Assumption 1}

In Section 2.3 of the main text, I characterize an equilibrium outcome in a directed search market by imposing the following assumption:

\section{Assumption 2}

(i) $w^{d}(\theta ; Q, \Psi)$ is continuous in $\theta$. 
(ii) For all $\theta \in \operatorname{Supp}(q), w^{d}, \mathcal{V}^{d}$, and $\mathcal{U}^{d}$ are given by (14), (15), and (16) respectively.

(iii) For all $\theta^{\prime}, \theta^{\prime \prime} \notin \operatorname{Supp}(q),\left|w^{d}\left(\theta^{\prime} ; Q, \Psi\right)-w^{d}\left(\theta^{\prime \prime} ; Q, \Psi\right)\right|<\left|\theta^{\prime}-\theta^{\prime \prime}\right|$.

In this section, I relax this assumption and characterize the equilibrium outcome(s) when both on-path and off-path wages are determined by Nash bargaining. I also show that relaxing Assumption 1 introduces a new trade-off for firms.

\section{B.0.1. On-path Sub-markets}

Nothing changes for on-path sub-markets; for all $\theta \in S u p p(q), w^{d}, \mathcal{V}^{d}$, and $\mathcal{U}^{d}$ are given by (14), (15), and (16) respectively. Additionally, firms must be indifferent between all on-path sub-markets, i.e., for all $\theta^{\prime}, \theta^{\prime \prime} \in \operatorname{Supp}(q), \mathcal{U}^{d}\left(\theta^{\prime} ; Q, \Psi\right)=\mathcal{U}^{d}\left(\theta^{\prime \prime} ; Q, \Psi\right)=\mathcal{U}^{d}(Q, \Psi)$. Note that for all on-path sub-markets, a firm's payoff satisfies

$$
\mathcal{U}^{d}(Q, \Psi) \leq \inf _{\theta \in \operatorname{Supp}(q)} \frac{\rho \theta}{\gamma+(1-\gamma) \rho}
$$

\section{B.0.2. Off-path Sub-markets}

For a sub-market $S(\theta)$ with $\theta \notin S u p p(q)$, the worker match probability is $\beta(\theta ; Q, \Psi)=0$, which implies $\mathcal{V}^{d}(\theta ; Q, \Psi)=0$. Suppose a firm deviates to an off-path sub-market $S(\theta)$ for one period. Wages are still determined by Nash bargaining but are now given by the solution to

$$
\begin{aligned}
w^{d}(\theta ; Q, \Psi) & =\underset{w \in \mathbb{R}}{\arg \max }\left(\theta-w-(1-\gamma) \mathcal{U}^{d}(Q, \Psi)\right)^{\rho}(w-0)^{1-\rho} \\
& =(1-\rho)\left(\theta-(1-\gamma) \mathcal{U}^{d}(Q, \Psi)\right)
\end{aligned}
$$

where $\mathcal{U}^{d}(Q, \Psi)$ is the firm's on-path payoff. Hence, the firm's one-shot deviation payoff is given by

$$
\mathcal{U}^{d}(\theta ; Q, \Psi)=\theta-(1-\rho)\left(\theta-(1-\gamma) \mathcal{U}^{d}(Q, \Psi)\right)
$$

In equilibrium, this one-shot deviation payoff must be (weakly) worse than the on-path payoff, i.e., for all $\theta \notin \operatorname{Supp}(q)$,

$$
\frac{\rho \theta}{\gamma+(1-\gamma) \rho} \leq \mathcal{U}^{d}(Q, \Psi)
$$




\section{B.0.3. Cutoff Strategies}

Suppose in equilibrium there exist sub-markets $S\left(\theta^{\prime}\right)$ and $S\left(\theta^{\prime \prime}\right)$ such that $\theta^{\prime}>\theta^{\prime \prime}, \theta^{\prime \prime} \in S u p p(q)$, and $\theta^{\prime} \notin \operatorname{Supp}(q)$. Then

$$
\frac{\rho \theta^{\prime}}{\gamma+(1-\gamma) \rho} \underbrace{\leq}_{\text {by }(\mathrm{B} 2)} \mathcal{U}^{d}(Q, \Psi) \underbrace{\leq}_{\text {by (B1) }} \frac{\rho \theta^{\prime \prime}}{\gamma+(1-\gamma) \rho}
$$

which is a contradiction. Hence, there exists some cutoff $\underline{\theta}_{d}$ such that $\theta \in \operatorname{Supp}(q)$ if $\theta>\underline{\theta}_{d}$ and $\theta \notin \operatorname{Supp}(q)$ if $\theta<\underline{\theta}_{d}$. Furthermore,

$$
\lim _{\theta \uparrow \underline{\theta}_{d}} \mathcal{U}^{d}(\theta ; Q, \Psi)=\frac{\rho \underline{\theta}_{d}}{\gamma+(1-\gamma) \rho}=\mathcal{U}^{d}(Q, \Psi) .
$$

Note that payoffs and match probabilities must be continuous on-path and at $\underline{\theta}_{d}$; otherwise the firms have a strict incentive to deviate. This implies that $\lim _{\theta \downarrow \underline{\theta}_{d}} \beta(\theta ; Q, \Psi)=0$ and $\lim _{\theta \downarrow \underline{\theta}_{d}} \alpha(\theta ; Q, \Psi)=1$. Additionally, $\beta(\theta ; Q, \Psi) \in(0,1)$ and $\alpha(\theta ; Q, \Psi)=1$ for $\theta$ in a right neighborhood of $\underline{\theta}_{d}$.

However, there must also exist another cutoff $\bar{\theta}_{d} \in\left(\underline{\theta}_{d}, 1\right)$ such that $\beta(\theta ; Q, \Psi)=1$ and $\alpha(\theta ; Q, \Psi) \in(0,1)$ for all $\theta>\bar{\theta}_{d}$. Otherwise, the firms would always be on the short-side of each on-path sub-market, violating the scarcity assumption. By continuity, $\beta\left(\bar{\theta}_{d} ; Q, \Psi\right)=$ $\alpha\left(\bar{\theta}_{d} ; Q, \Psi\right)=1$, and by $(16)$,

$$
\mathcal{U}^{d}\left(\bar{\theta}_{d} ; Q, \Psi\right)=\frac{\rho \alpha\left(\bar{\theta}_{d} ; Q, \Psi\right) \bar{\theta}_{d}}{\gamma+(1-\gamma)\left(\rho \alpha\left(\bar{\theta}_{d} ; Q, \Psi\right)+(1-\rho) \beta\left(\bar{\theta}_{d} ; Q, \Psi\right)\right)}=\rho \bar{\theta}_{d}
$$

Since $\bar{\theta}_{d} \in \operatorname{Supp}(q)$, we also have $\mathcal{U}^{d}\left(\bar{\theta}_{d} ; Q, \Psi\right)=\mathcal{U}^{d}(Q, \Psi)=\frac{\rho \underline{\theta}_{d}}{\gamma+(1-\gamma) \rho}$. Thus,

$$
\underline{\theta}_{d}=\bar{\theta}_{d}(\gamma+(1-\gamma) \rho)
$$

\section{B.0.4. Match Probabilities}

For off-path sub-markets, the firm match probability is always 1 while the worker match probability is always 0. For on-path sub-markets, the match probabilities must be calibrated in such a way that the firms are indifferent among all the on-path sub-markets.

For each sub-market $S(\theta)$ with $\theta \in\left(\underline{\theta}_{d}, \bar{\theta}_{d}\right), \alpha(\theta ; Q, \Psi)=1$. From (16) and (B3), the 
on-path indifference condition $\mathcal{U}^{d}(\theta ; Q, \Psi)=\mathcal{U}^{d}(Q, \Psi)$ can be expressed as

$$
\begin{aligned}
\frac{\rho \theta}{\gamma+(1-\gamma)(\rho+(1-\rho) \beta(\theta ; Q, \Psi))} & =\frac{\rho \underline{\theta}_{d}}{\gamma+(1-\gamma) \rho} \\
\Longrightarrow \beta(\theta ; Q, \Psi) & =\frac{\theta-\underline{\theta}_{d}}{\bar{\theta}_{d}(1-\gamma)(1-\rho)} .
\end{aligned}
$$

Similarly, for each sub-market $S(\theta)$ with $\theta \in\left(\underline{\theta}_{d}, 1\right], \beta(\theta ; Q, \Psi)=1$. Thus, the on-path indifference condition $\mathcal{U}^{d}(\theta ; Q, \Psi)=\mathcal{U}^{d}(Q, \Psi)$ can be expressed as

$$
\begin{aligned}
\frac{\rho \alpha(\theta ; Q, \Psi) \theta}{\gamma+(1-\gamma)(\rho \alpha(\theta ; Q, \Psi)+1-\rho)} & =\frac{\rho \underline{\theta}_{d}}{\gamma+(1-\gamma) \rho} \\
\Longrightarrow \alpha(\theta ; Q, \Psi) & =\frac{\bar{\theta}_{d}(1-\rho(1-\gamma))}{\theta-\bar{\theta}_{d} \rho(1-\gamma)}
\end{aligned}
$$

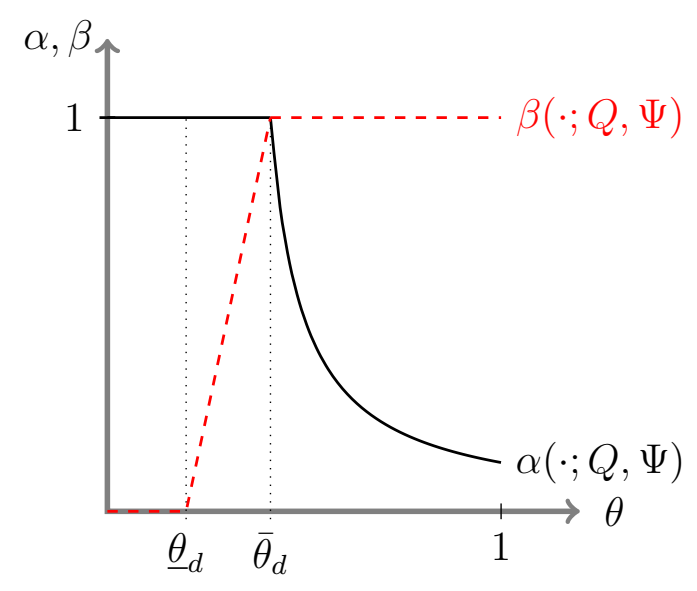

Figure 4: The dashed red curve line plots the worker match probabilities while the solid black curve depicts the firm match probability. 


\section{B.0.5. Market Composition and Queueing CDF}

The market composition can now easily be derived from (3)-(5) in Lemma 1 with

$$
\ell(\theta)=\beta(\theta ; Q, \Psi)=\left\{\begin{array}{ccc}
0 & \text { if } & \theta \leq \underline{\theta}_{d} \\
\frac{\theta-\underline{\theta}_{d}}{\bar{\theta}_{d}(1-\gamma)(1-\rho)} & \text { if } & \theta \in\left[\underline{\theta}_{d}, \bar{\theta}_{d}\right] \\
1 & \text { if } & \theta \geq \bar{\theta}_{d}
\end{array}\right.
$$

In particular, we have

$$
\boldsymbol{M}^{a}(\ell)=\frac{1}{\gamma}\left(k_{a}-k_{o}(1-\gamma)\left(1-F\left(\bar{\theta}_{d}\right)\right)-k_{o} \int_{\underline{\theta}_{d}}^{\bar{\theta}_{d}} \frac{\omega-\underline{\theta}_{d}}{\omega-\bar{\theta}_{d} \rho} d F(\theta)\right) .
$$

However, the inflow-outflow of firms can also be expressed as

$$
\begin{aligned}
k_{a} & =\boldsymbol{M}^{a}(\ell) \int_{\underline{\theta}_{d}}^{1}(\alpha(\omega ; Q, \Psi)(1-\gamma)+\gamma) d Q(\omega) \\
& =\boldsymbol{M}^{a}(\ell) \int_{\bar{\theta}_{d}}^{1}\left(\frac{\boldsymbol{M}^{o}(\ell) \boldsymbol{g}(\omega ; \ell)}{\boldsymbol{M}^{a}(\ell) q(\omega)}(1-\gamma)+\gamma\right) q(\omega) d \omega+\boldsymbol{M}^{a}(\ell)\left(Q\left(\bar{\theta}_{d}\right)-Q\left(\underline{\theta}_{d}\right)\right) \\
& =k_{o}(1-\gamma)\left(1-F\left(\bar{\theta}_{d}\right)\right)+\boldsymbol{M}^{a}(\ell)\left(\gamma+Q\left(\bar{\theta}_{d}\right)(1-\gamma)\right)
\end{aligned}
$$

where the second line follows because $\alpha(\theta ; Q, \Psi)=1$ for $\theta \in\left(\underline{\theta}_{d}, \bar{\theta}_{d}\right)$ and $\alpha(\theta ; Q, \Psi)=\frac{M^{o} g(\theta)}{M^{a} q(\theta)}<1$ for $\theta \in\left(\bar{\theta}_{d}, 1\right]$, and the last line follows from (3)-(4) and $Q\left(\underline{\theta}_{d}\right)=0 .{ }^{29}$ The expression implies

$$
\boldsymbol{M}^{a}(\ell) Q\left(\bar{\theta}_{d}\right)=\frac{k_{o}}{1-\gamma} \int_{\underline{\theta}_{d}}^{\bar{\theta}_{d}} \frac{\omega-\underline{\theta}_{d}}{\omega-\bar{\theta}_{d} \rho} d F(\theta)
$$

\section{B.0.6. Equilibrium Characterization}

For all $\theta>\bar{\theta}_{d}$, firms are on the long-side of the sub-market, i.e, there is congestion. Hence,

$$
\alpha(\theta ; Q, \Psi)=\min \left\{\frac{\boldsymbol{M}^{o}(\ell) \boldsymbol{g}(\theta ; \ell)}{\boldsymbol{M}^{a}(\ell) q(\theta)}, 1\right\}=\frac{\boldsymbol{M}^{o}(\ell) \boldsymbol{g}(\theta ; \ell)}{\boldsymbol{M}^{a}(\ell) q(\theta)}=\frac{k_{o} f(\theta)}{\boldsymbol{M}^{a}(\ell) q(\theta)} .
$$

\footnotetext{
${ }^{29}$ Recall that off-path markets are $\theta<\underline{\theta}_{d}$.
} 
From (B6), we have

$$
\frac{k_{o} f(\theta)}{\boldsymbol{M}^{a}(\ell) q(\theta)}=\frac{\bar{\theta}_{d}(1-\rho(1-\gamma))}{\theta-\bar{\theta}_{d} \rho(1-\gamma)} .
$$

Rearranging and integrating over the interval $\left(\bar{\theta}_{d}, 1\right]$ gives

$$
\bar{\theta}_{d}\left((1-\rho(1-\gamma)) \boldsymbol{M}^{a}(\ell)\left(1-Q\left(\bar{\theta}_{d}\right)\right)+\rho k_{o}(1-\gamma)\left(1-F\left(\bar{\theta}_{d}\right)\right)\right)=k_{o} \int_{\bar{\theta}_{d}}^{1} \theta d F(\theta) .
$$

Replacing $\boldsymbol{M}^{a}(\ell)$ and $\boldsymbol{M}^{a}(\ell) Q\left(\bar{\theta}_{d}\right)$ with (B7) and (B8) respectively, we have

$$
\begin{aligned}
\bar{\theta}_{d} & =\frac{k_{o} \gamma}{k_{a}(1-\rho(1-\gamma))-k_{o}(1-\rho)(1-\gamma)\left(1-F\left(\bar{\theta}_{d}\right)\right)} \int_{\bar{\theta}_{d}}^{1} \omega d F(\omega) \\
& +\left(\frac{\bar{\theta}_{d}}{1-\gamma}\right)\left(\frac{k_{o}(1-\rho(1-\gamma))}{k_{a}(1-\rho(1-\gamma))-k_{o}(1-\rho)(1-\gamma)\left(1-F\left(\bar{\theta}_{d}\right)\right)}\right) \int_{\underline{\theta}_{d}}^{\bar{\theta}_{d}} \frac{\omega-\underline{\theta}_{d}}{\omega-\bar{\theta}_{d} \rho} d F(\omega) \\
& \triangleq \mathbb{D}\left(\bar{\theta}_{d}\right) .
\end{aligned}
$$

Notice that $\mathbb{D}$ is only a function of $\bar{\theta}_{d}$ because we can use (B4) to express $\underline{\theta}_{d}$ in terms of $\bar{\theta}_{d}$. It is straightforward to check that $\mathbb{D}$ is a continuous function with

$$
\mathbb{D}(0)=\frac{k_{o} \gamma}{k_{a}(1-\rho(1-\gamma))-k_{o}(1-\rho)(1-\gamma)} \mathbb{E}_{F}[\theta]>0
$$

and

$$
\mathbb{D}(1) \leq \frac{k_{o}}{k_{a}}(1-F(\gamma+(1-\gamma) \rho))<1 .
$$

Hence, by the intermediate value theorem, there is at least one $\bar{\theta}_{d}$ such that $\bar{\theta}_{d}=\mathbb{D}\left(\bar{\theta}_{d}\right)$ which establishes existence of an equilibrium. However, the equilibrium need not be unique.

The firms can settle and hire a worker for which there is little competition. The cutoff $\bar{\theta}_{d}$ is the highest productivity type any firm can hire without entering a congested sub-market. Hence, the LHS of (B9) is the value of settling.

In equilibrium, the firms equate the value of settling with the value of search. The on-path sub-markets are now divided into thin sub-markets $\cup_{\underline{\theta}_{d}<\theta<\bar{\theta}_{d}} S(\theta)$ in which the firms are on the short-side, and congested sub-markets $\cup_{\theta>\theta_{d}} S(\theta)$ in which the firms are on the long-side. The first term on the RHS of (B9) is the value of searching in a congested sub-market for a 
worker with productivity higher than $\bar{\theta}_{d}$. Notice that this first term is the same as (17). Hence, the equilibrium characterization without Assumption 1 is similar to the characterization in Proposition 4 except there is now an additional second term capturing the value of search in a thin sub-market.

The workers in the thin sub-markets are less productive than $\bar{\theta}_{d}$ and one may expect the firms to not be interested in these workers. However, the workers in the thin sub-markets are at a disadvantage when they bargain with firms because if they refuse a wage offer, they may not be matched again in the next period. Thus, they are willing to accept low wage offers. Therefore, a firm's total value of search is a combination of the value of searching for a productive worker by waiting in a congested sub-market and the value of hiring a less productive worker for a low wage.

In general, it is not obvious (at least to me) whether random search or directed search yields a higher welfare. However, notice that when $\gamma$ or $\rho$ equal $1, \underline{\theta}_{d}=\bar{\theta}_{d}$, and there is a unique directed search SME characterized by

$$
\bar{\theta}_{d}=\frac{k_{o}}{k_{a}} \int_{\bar{\theta}_{d}}^{1} \omega d F(\omega) .
$$

Since $\mathbb{D}$ is continuous in both $\gamma$ and $\rho$, all fixed points solving (B9) converge to the unique fixed point solving (B10) (by upper hemi-continuity) as either $\rho$ or $\gamma$ converge to 1 . Thus, we can conclude that the main result of Theorem 2-random search with ex-post transfers is more efficient than directed search with ex-post transfers - continues to hold whenever $(1-\rho)(1-\gamma)$ is close to 0 .

\section{References}

Acemoglu, D. and Shimer, R. . Holdups and efficiency with search frictions. International Economic Review, 40(4):827-849, 1999a.

Acemoglu, D. and Shimer, R. . Efficient unemployment insurance. Journal of political Economy, 107(5):893-928, 1999b.

Arnosti, N. , Johari, R. , and Kanoria, Y. . Managing congestion in matching markets. 2015.

Burdett, K. and Coles, M. G. . Marriage and class. The Quarterly Journal of Economics, 112 (1):141-168, 1997. 
Burdett, K. and Menzio, G. . The (q, s, s) pricing rule. The Review of Economic Studies, 85 (2):892-928, 2017.

Butters, G. R. . Equilibrium distributions of sales and advertising prices. The Review of Economic Studies, 44(3):465-491, 1977.

Coles, P. , Kushnir, A. , and Niederle, M. . Preference signaling in matching markets. American Economic Journal: Microeconomics, 5(2):99-134, 2013.

Dall, T., West, T., Chakrabarti, R., Reynolds, R., and Iacobucci, W. . 2018 update the complexities of physician supply and demand: Projections from 2016 to 2030 final report association of american medical colleges. 03 2018. doi: 10.13140/RG.2.2.25694.48963.

Davis, S. J. . The quality distribution of jobs and the structure of wages in search equilibrium. Technical report, National Bureau of Economic Research, 2001.

Diamond, P. A. . Wage determination and efficiency in search equilibrium. The Review of Economic Studies, 49(2):217-227, 1982.

Eeckhout, J. and Kircher, P. . Sorting and decentralized price competition. Econometrica, 78 (2):539-574, 2010.

Gale, D. . Limit theorems for markets with sequential bargaining. Journal of Economic Theory, 43(1):20-54, 1987.

Halaburda, H. , Jan Piskorski, M. , and Yıldırım, P. . Competing by restricting choice: The case of matching platforms. Management Science, 2017.

Hirshleifer, J. . The private and social value of information and the reward to inventive activity. The American Economic Review, 61(4):561-74, 1971.

Inderst, R. . Matching markets with adverse selection. Journal of Economic Theory, 121(2): 145-166, 2005.

Kanoria, Y. and Saban, D. . Facilitating the search for partners on matching platforms: Restricting agents' actions. 2017.

Kim, K. and Kircher, P. . Efficient competition through cheap talk: the case of competing auctions. Econometrica, 83(5):1849-1875, 2015. 
Lauermann, S. Asymmetric information in bilateral trade and in markets: An inversion result. Journal of Economic Theory, 147(5):1969-1997, 2012.

Lauermann, S. . Dynamic matching and bargaining games: A general approach. American Economic Review, 103(2):663-89, 2013.

Lester, B. , Shourideh, A., Venkateswaran, V. , and Zetlin-Jones, A. . Screening and adverse selection in frictional markets. Working Papers 16-10, Federal Reserve Bank of Philadelphia, March 2016. URL https://ideas.repec.org/p/fip/fedpwp/16-10.html.

McCall, J. J. . Economics of information and job search. The Quarterly Journal of Economics, pages $113-126,1970$.

Mekonnen, T. . Random versus directed search for scarce resources: Online appendix. 2018. URL https://papers.ssrn.com/sol3/papers.cfm?abstract_id=3284990.

Menzio, G. . A cheap-talk theory of random and directed search. Manscript, Univ. Pennsylvania, 2005.

Menzio, G. . A theory of partially directed search. Journal of political Economy, 115(5):748-769, 2007.

Moen, E. R. . Competitive search equilibrium. Journal of political Economy, 105(2):385-411, 1997.

Montgomery, J. D. . Equilibrium wage dispersion and interindustry wage differentials. The Quarterly Journal of Economics, 106(1):163-179, 1991.

Mortensen, D. T. . The matching process as a noncooperative bargaining game. In The economics of information and uncertainty, pages 233-258. University of Chicago Press, 1982.

Mortensen, D. T. and Wright, R. . Competitive pricing and efficiency in search equilibrium. International economic review, 43(1):1-20, 2002.

Olszewski, W. and Wolinsky, A. . Search for an object with two attributes. Journal of Economic Theory, 161:145-160, 2016.

Peters, M. . Ex ante price offers in matching games non-steady states. Econometrica: Journal of the Econometric Society, pages 1425-1454, 1991. 
Peters, M. . On the equivalence of walrasian and non-walrasian equilibria in contract markets: the case of complete contracts. The Review of Economic Studies, 64(2):241-264, 1997.

Peters, M. . Limits of exact equilibria for capacity constrained sellers with costly search. Journal of Economic Theory, 95(2):139-168, 2000.

Petrongolo, B. and Pissarides, C. A. . Looking into the black box: A survey of the matching function. Journal of Economic literature, 39(2):390-431, 2001.

Pissarides, C. A. . Short-run equilibrium dynamics of unemployment, vacancies, and real wages. The American Economic Review, 75(4):676-690, 1985.

Rogerson, R. , Shimer, R., and Wright, R. . Search-theoretic models of the labor market: A survey. Journal of economic literature, 43(4):959-988, 2005.

Satterthwaite, M. and Shneyerov, A. . Dynamic matching, two-sided incomplete information, and participation costs: Existence and convergence to perfect competition. Econometrica, $75(1): 155-200,2007$.

Satterthwaite, M. and Shneyerov, A. . Convergence to perfect competition of a dynamic matching and bargaining market with two-sided incomplete information and exogenous exit rate. Games and Economic Behavior, 63(2):435-467, 2008.

Schlee, E. E. . The value of information in efficient risk-sharing arrangements. American Economic Review, 91(3):509-524, 2001.

Shi, S. . A directed search model of inequality with heterogeneous skills and skill-biased technology. The Review of Economic Studies, 69(2):467-491, 2002.

Shimer, R. . The assignment of workers to jobs in an economy with coordination frictions. Journal of Political Economy, 113(5):996-1025, 2005. 